\title{
Water Quality in the
}

New River from Calexico to the Salton Sea Imperial County, California

United States Geological Survey Water-Supply Paper 2212

Prepared in cooperation with the California Department of Water Resources

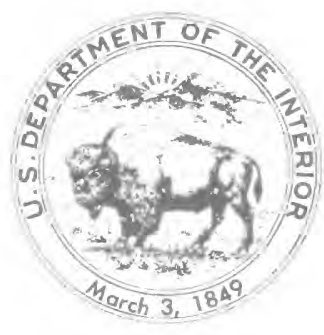




\section{Water Quality in the New River from Calexico to the Salton Sea Imperial County, California}

BY JAMES G. SETMIRE

Prepared in cooperation with the California

Department of Water Resources 


\title{
UNITED STATES DEPARTMENT OF THE INTERIOR
}

WILLIAM P. CLARK, Secretary

\author{
U.S. GEOLOGICAL SURVEY \\ Dallas L. Peck, Director
}

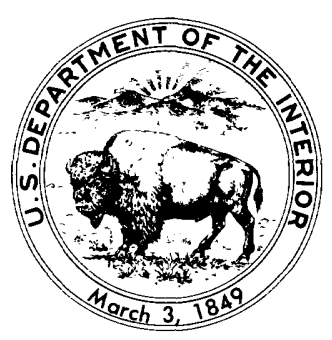

\section{Library of Congress cataloging in Publication Data}

Setmire, James G.

Water quality in the New River from Calexico to the Salton Sea, Imperial County, California.

(Geological Survey water-supply paper ; 2212)

Supt. of Docs. no.: I 19.13:2212

Bibliography: $42 \mathrm{p}$.

1. Water quality-New River (Mexico and Calif.) 2. Water quality-California. I. California. Dept. of Water Resources. II. Title. III. Series: U.S. Geological Survey water-supply paper ; 2212.

$\begin{array}{lll}\text { TD224.C3S45 } 1984 & 363.7^{\prime} 3942 \text { '0979499 }\end{array}$

$82-600195$

For sale by the Branch of Distribution

U.S. Geological Survey

604 South Pickett Street

Alexandria, VA 22304 


\title{
CONTENTS
}

\author{
Abstract 1 \\ Introduction 1 \\ Background 1 \\ Purpose and scope $\mathbf{5}$ \\ Field and laboratory procedures 6 \\ Reconnaissance 6 \\ Data collection 6 \\ Results 7 \\ Comparisons 9 \\ Water-quality investigations $\mathbf{1 1}$ \\ Pesticides and trace elements 11 \\ Dissolved oxygen 11 \\ Temperature effects $\mathbf{1 2}$ \\ Sag curves (profiles) 12 \\ Diel variations 17 \\ EPA criteria 19 \\ Organic material $\mathbf{2 0}$ \\ Oxidation of organics \\ Reaction rates 21 \\ Time-of-travel investigations 22 \\ Longitudinal stream profiles $\mathbf{2 2}$ \\ Diel measurements 24 \\ Loads 25 \\ Biota as indicators 26 \\ Benthic invertebrates 27 \\ Phytoplankton 33 \\ Bacteria 35 \\ Potential hazard $\mathbf{3 5}$ \\ Observed counts $\mathbf{3 8}$ \\ Summary and conclusions $\mathbf{3 8}$ \\ Selected references $\mathbf{4 2}$
}

\section{FIGURES}

1-2. Maps showing:

1. Location of study area 2

2. Location of sampling sites 3

3-4. Photographs showing:

3. Debris buildup on upstream side of box culvert at Interstate 8 road crossing 4

4. New River valley at Keystone Road crossing $\mathbf{5}$

5. Graph showing dissolved-oxygen concentration, percent saturation, $\mathrm{pH}$, and specific conductance in the New River at Calexico, May 12, 19778

6. Photograph showing New River at Calexico gaging station 9

7-8. Graphs showing dissolved-oxygen concentration at selected sites on the New River:

7. December 28, 1976, January 1, and April 5, $1977 \quad 18$

8. June 13, July 28, and August 30, 1977 


\section{FIGURES}

9. Photograph showing New River upstream of Westmorland gage during longitudinal profiling by boat September 26-30, $1977 \quad 22$

10. Graph showing dissolved-oxygen concentration and percent saturation in the New River from Calexico to the Westmorland gage, September 26-30, 197723

11-13. Graphs showing dissolved-oxygen concentration, percent saturation, and selected chemical constituents:

11. New River at Calexico, October 17-18, $1977 \quad 26$

12. New River at Highway 80, October 17-18, 197727

13. New River at Calexico, April 17-19, 197828

14. Graph showing diel (24-hour) dissolved-oxygen concentration and percent saturation at five sites on the New River and the control site on the Alamo River, April 17-21, $1978 \quad 29$

15. Graph showing distribution of concentrations of chemical oxygen demand and biochemical oxygen demand, New River at Calexico, July 1976 to March 197830

16. Graphs showing time of travel of dye and discharge in the New River from Calexico to Westmorland gage February 6-9, and June 19-22, 197832

17. Graph showing dissolved-oxygen concentration, percent saturation, and selected chemical constituents at selected sites on the New River from Calexico to the Westmorland gage, May 10, 197834

18. Graph showing number of benthic invertebrate species and organisms for selected sites on the New River and the control site on the Alamo River 35

19. Graph showing number of phytoplankton genera and organisms for selected sites on the New River and the control site on the Alamo River 38

\section{TABLES}

1. Field measurements of selected physical and chemical characteristics, New River at Calexico, May 9-13, 19777

2. Summary of water-quality field data, New River at Calexico, May 9-13, 19777

3. Laboratory analyses of water, New River at Calexico, May 10-12, 197710

4. Field measurements and laboratory analyses of water at control site, Alamo River at Drop 9 near Holtville, May 12, 197711

5. Comparison of water-quality constituents during periods of background and slug flow conditions 11

6. Chemical and physical analyses of water, 1978-79, at selected sites $\mathbf{1 2}$

7. Field measurements of selected physical and chemical characteristics at sites on the New River, September 26-30, 197724

8. Time-of-travel data for selected sites on the New River, February 6-9, and June 19-22, 197831

9. Determinations of selected physical, chemical, and biological characteristics at sites on the New River, September 26-30, 197733

10. Loads of selected constituents for the composite sampling program $\mathbf{3 5}$

11. Phyla and genera of benthic invertebrate samples, from the New and Alamo Rivers 36

12. Phyla and genera of phytoplankton samples from the New and Alamo Rivers 


\title{
Water Quality in the New River From Calexico to the Salton Sea, Imperial County, California
}

\author{
By James G. Setmire
}

\begin{abstract}
The New River enters the United States at Calexico, Calif., after it crosses the international boundary. Water-quality data from routine collection indicated that the New River was degraded by high organic and bacterial content. Intensive sampling for chemical and physical constituents and properties of the river was done May 9-13, 1977, to quantify the chemical composition of the water and to identify water-quality problems. Concentrations of total organic carbon in the New River at Calexico ranged from 80 to 161 milligrams per liter and dissolved organic carbon ranged from 34 to 42 milligrams per liter; the maximum chemical oxygen demand was $\mathbf{5 1 0}$ milligrams per liter. Intensive sampling for chemical and biological characteristics was done in the New River from May 1977 to June 1978 to determine the occurrence of the organic material and its effects on downstream water quality.
\end{abstract}

Dissolved-oxygen concentration was measured along longitudinal profiles of the river from Calexico to the Salton Sea. A dissolved-oxygen sag downstream from the Calexico gage varied seasonally. The sag extended farther downstream and had lower concentrations of dissolved oxygen during the summer months than during the winter months. The sag of zero dissolved-oxygen concentration extended 26 miles in July 1977. In December 1976, the sag extended 20 miles but the minimum dissolved-oxygen concentration was 2.5 milligrams per liter. The greatest diel (24-hour) variation in dissolved-oxygen concentration occurred in the reach from the Calexico gage to Lyons Crossing, 8.8 miles downstream. High concentrations of organic material were detected as far as Highway 80, 19.5 miles downstream from the international boundary.

Biological samples analyzed for benthic invertebrates showed that water at the Calexico and Lyons Crossing sites, nearest the international boundary, was of such poor quality that very few bottom-dwelling organisms could survive. Although the water was of poor quality at Keystone Road, 36 miles downstream, it was able to support a benthic community. The April sample had more than 9,150 organisms on a multiplate sampler, 8,770 of which were of one species. Farther downstream at the Westmorland gage, the water quality, as indicated by the number and diversity of organisms, had improved over that at the Keystone site. The Alamo River at its outlet to the Salton Sea-the control site-had the greatest diversity of all the study sites. This diversity, when compared with the diversity at the Westmorland gage, indicated that the effects of the degraded water quality observed at the New River at Calexico are detected as far as 62 miles downstream. Standard bacteria indicator tests indicate that fecal contamination exists in the New River. Counts of fecal coliform bacteria ranged from 180,000 to $2,800,000$ colonies per 100 milliliters for the 20-mile reach from Calexico to Highway 80 , and fecal streptococcal bacteria ranged from 5,000 to 240,000 colonies per $\mathbf{1 0 0}$ milliliters.

\section{INTRODUCTION}

Water entering the United States at Calexico, Calif., creates major problems in the New River. Water quality in the New River is also influenced by numerous point and nonpoint discharges along its 60 -mile course in the United States from Calexico, Calif., to Westmorland (figs. 1, 2, 3, and 4).

Visual observation of the water at the Calexico gage indicates serious water-quality problems. For example, the following are remarks taken from sampling notes: (1) "July $28,1976,1550$ to 1700 hours, slug flow of white discharge; white particulate matter on surface; thousands of dead and dying minnows observed; dissolved oxygen zero;" and (2) "June 1, 1977, 1105 hours, slug flow of white discharge; dead and dying minnows on surface; strong sewage odor." These are but a few of the many observations of water-quality problems and associated fish kills in the New River at Calexico. Conditions downstream are similar. At La Boucherie Road, May 10, 1977 , at 1800 hours, the dissolved-oxygen (DO) concentration was zero, the river was black, a sulfide odor was present, and dead fish were observed. The same kinds of observations have been made at Drew, Brockman, Lyons, and Clark Roads throughout the last few years.

\section{Background}

The New River flows northward from the Colorado River Delta in the Mexicali Valley of Mexico, across the international boundary at Calexico, through the Imperial Valley, and into the Salton Sea (figs. 1 and 2). The Imperial Valley is bordered on the northwest by the Salton 


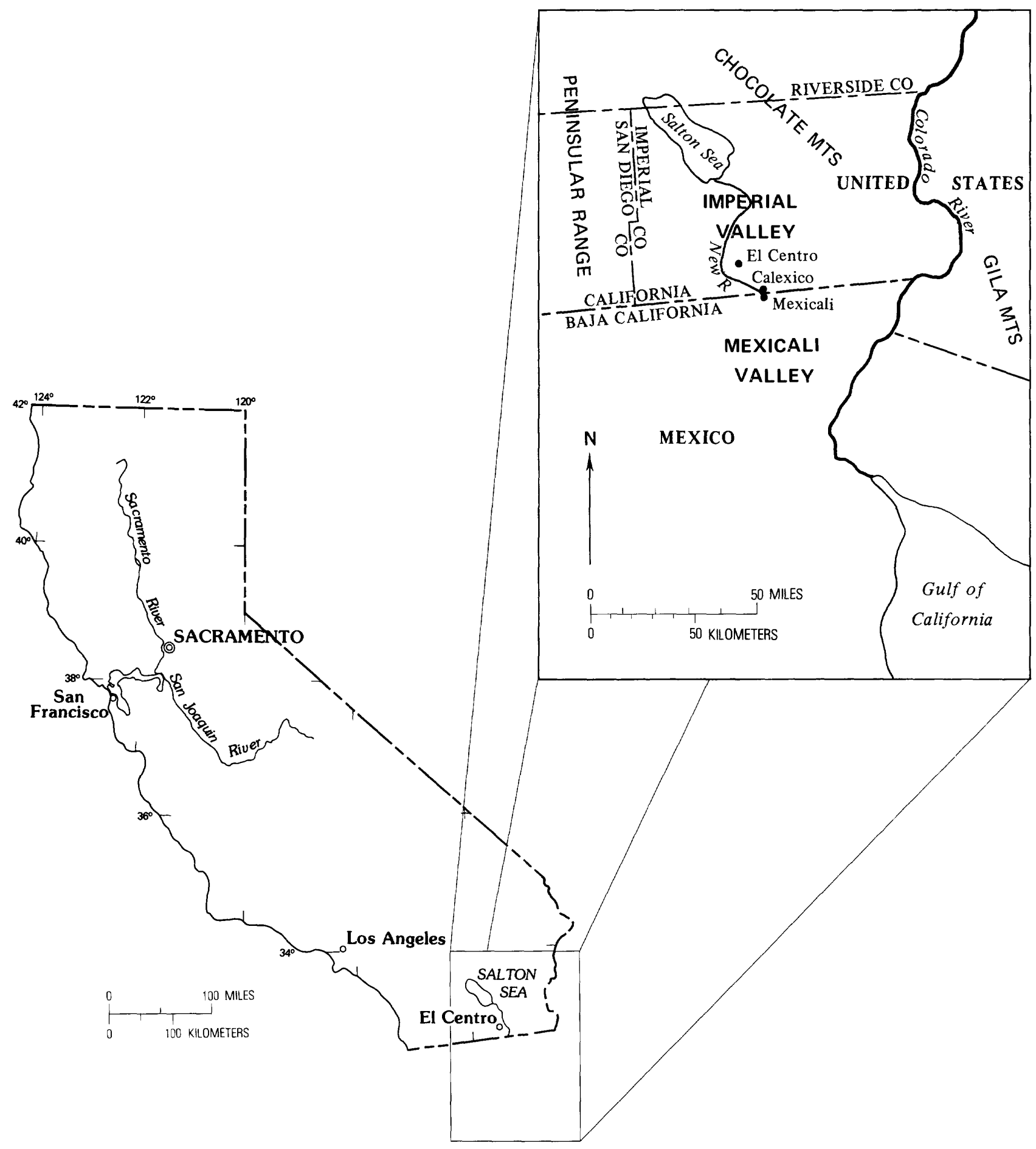

Figure 1. Location of study area.

Sea, on the northeast by the Chocolate Mountains, and on the southwest by the Peninsular Range of southern California and Baja California. On the southeast it is contiguous with the Mexicali Valley of Mexico.

The New River was formed in the 1840's when the Colorado River abandoned its normal course and flowed northward. This flow was seasonal and did not provide a constant source of water for the Imperial Valley. In 1853, an exploration to find a railroad route in California involved a survey of the Imperial Valley area. A report by geologist William P. Blake (1857) included the following statements: "The New River and its lagoons cannot be relied upon as an unfailing source of water; it depends upon the Colorado being filled during the season of 


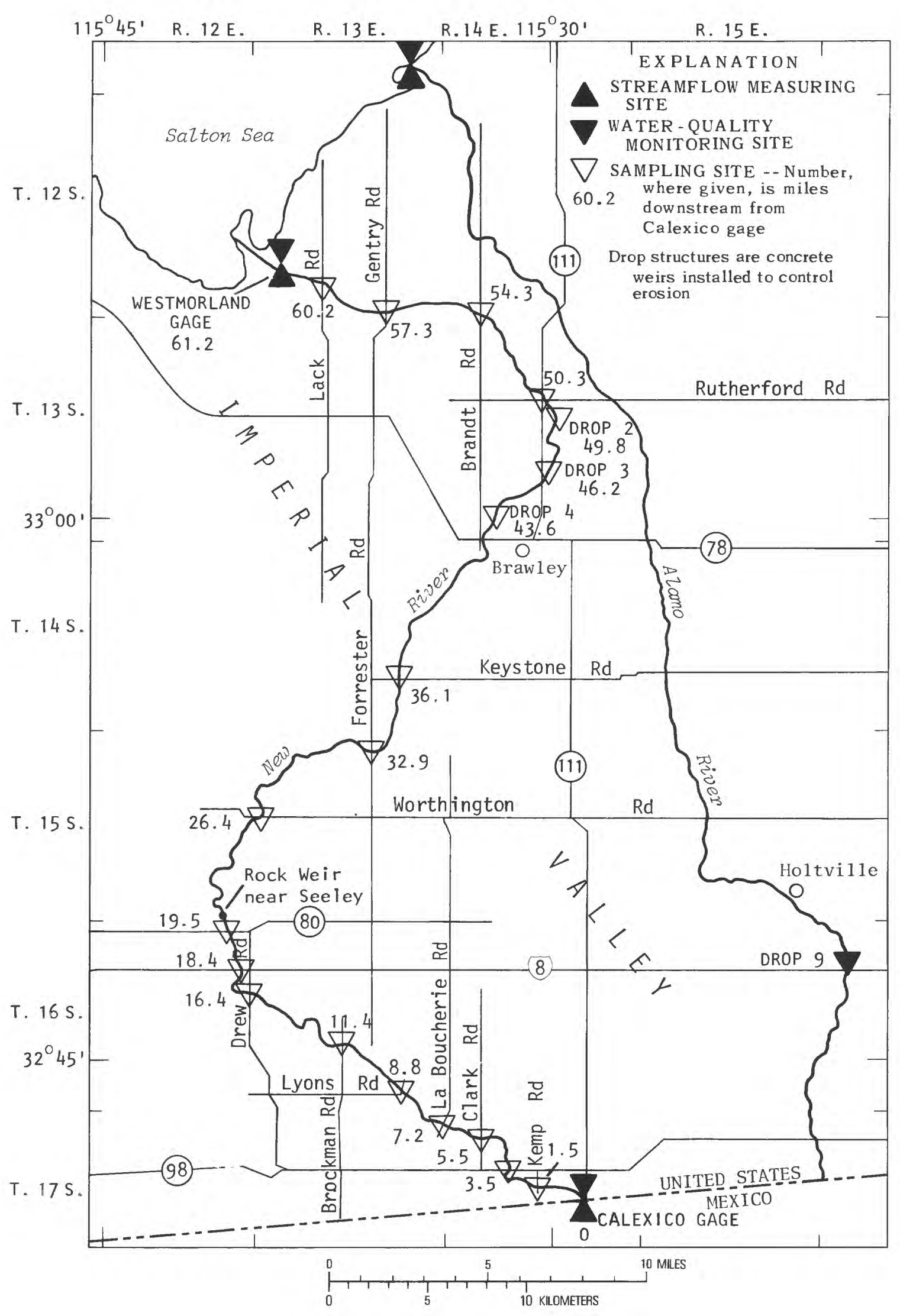

Figure 2. Location of sampling sites. 


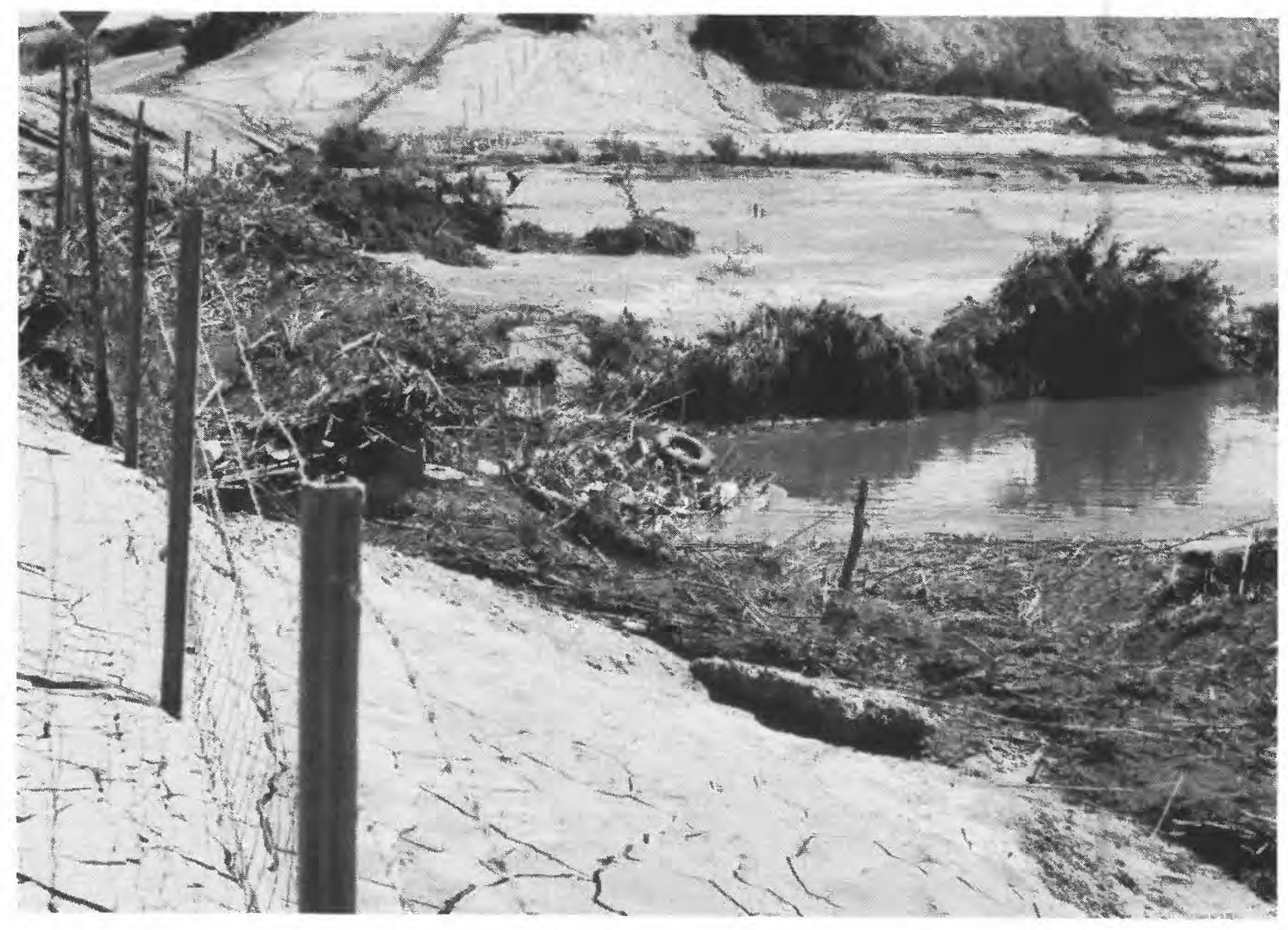

Figure 3. Debris buildup on upstream side of box culvert at Interstate 8 road crossing.

floods, and not then unless the river attains unusual height." At that time, the New River did not flow as far north as today's international boundary, and the present Salton Sea had not been formed. In the middle 1800's, water flowed into the Salton Sink on four occasions: $1840,1842-52,1859$, and 1867. In 1891, water from the Colorado River flowed in the New River and formed a lake of 100,000 acres in the Salton Sink. This water evaporated leaving a salt marsh. The marsh was inundated in 1906 when a break in a diversion of the Colorado River caused the entire discharge of the river into the Salton Sink for 4 months, thereby forming the current Salton Sea.

In 1853, the New River did not flow continuously across today's international boundary. In 1975 the discharge records show that the river's flow was continuous with more than 100,000 acre- $\mathrm{ft} / \mathrm{yr}$ at the international boundary, receiving more than 300,000 acre-ft/yr of irrigation return flow in the United States. Irrigation return water contains lower concentrations of dissolved solids than does the water crossing the international boundary. As a result, this irrigation return improves the water qual- ity as measured by the dissolved-solids concentration. Also, irrigation return apparently contains lower concentrations of organic materials than water crossing the international boundary, so that water quality as measured by organic concentration also improves. Nevertheless, some problems associated with high concentrations of organic material are:

1. Sudden shifts to anaerobic conditions, resulting in numerous fish kills in the first $20 \mathrm{mi}$ below the boundary.

2. An overall lowering of the DO concentration of the entire river, especially in its upper reaches, rendering it unfit for most fish populations and changing the environment of the river to less desirable biota and a poorer esthetic quality.

3. Eutrophication, algae blooms, and fish kills in the Salton Sea, reducing its desirability as a recreation area.

The New River and the Salton Sea are major feeding areas for migratory birds, and the Salton Sea is a spawning and rearing area for several species of gamefish. In 1965-67, 357,000 fisherman days per year were spent on the Salton Sea (U.S. Department of the Interior and 


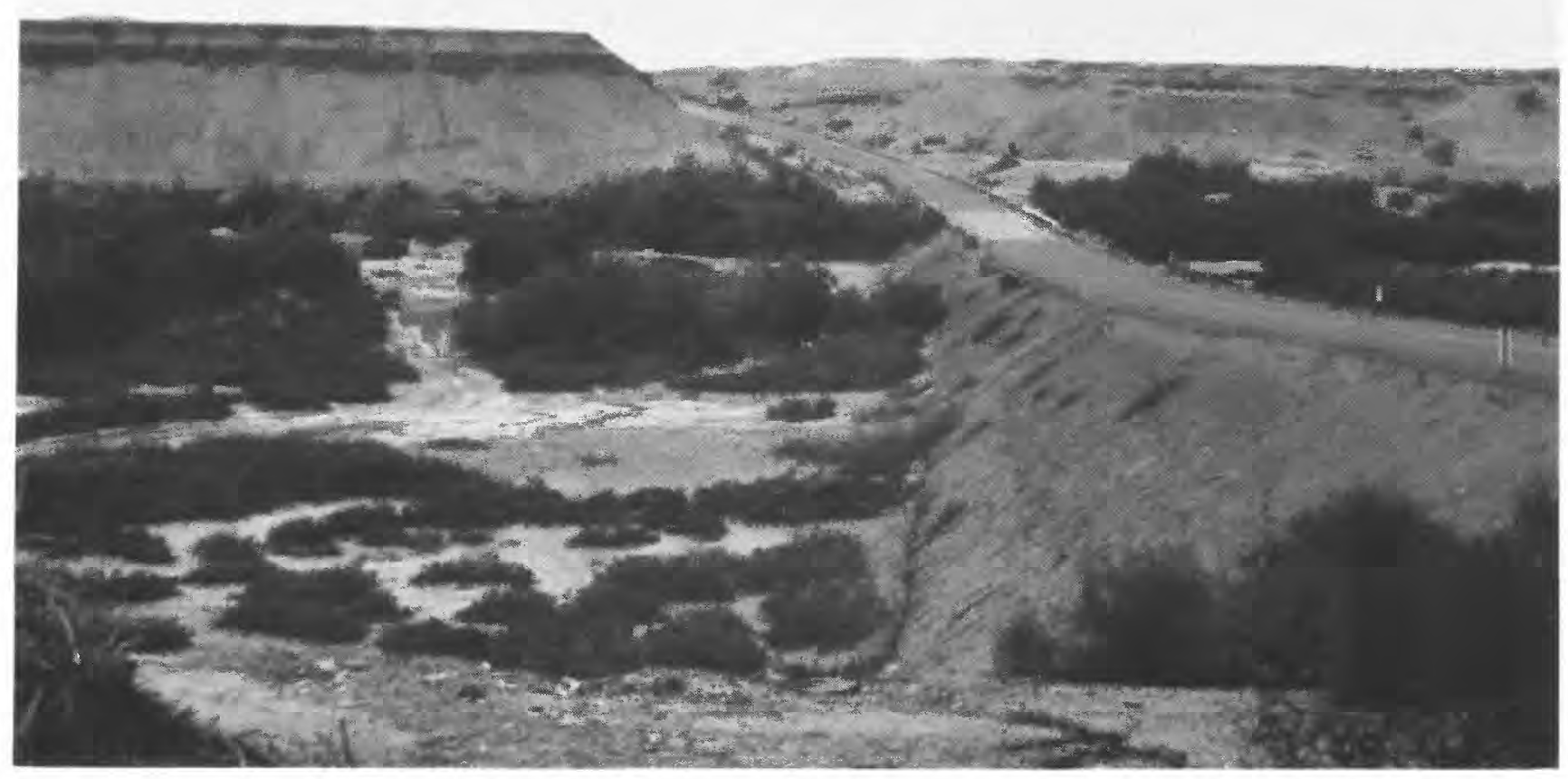

Figure 4. New River valley at Keystone Road crossing.

the Resources Agency of California, 1974). The three main gamefish in the sea are the orangemouth corvina (Cynoscion xanthulus), the gulf croaker (Bairdiella icistia), and the sargo (Anisotremus davidsoni). Migratory birds, such as snow geese and Canada geese, use Salton Sea areas during their annual journeys, while delta areas of the New and Alamo Rivers afford a habitat for the Yuma clapper rail and the California brown pelican, both of which have been placed on the endangered species list. The tip of the New River Delta is the only nesting area in the Western United States for the gull-billed tern. An egret rookery is also present in the south end of the Salton Sea, as are food plots to induce waterfowl away from the agricultural areas. The food plots are in the 2,200-acre Salton Sea National Wildlife Refuge (U.S. Department of the Interior and the Resources Agency of California, 1974).

\section{Purpose and Scope}

The purposes of this study were (1) to identify the major constituents degrading the quality of the water in the New River at Calexico, (2) to document existing water-quality conditions in the New River from Calexico to the Salton Sea, (3) to describe the effects of the water quality at Calexico on the quality of the river as it flows to the Salton Sea, and (4) to document the daily loads of selected constituents crossing the international boundary.

A reconnaissance study of water quality was made to confirm visual observations by quantifying the chemical composition of the water and by identifying the major problems. The reconnaissance study also showed what was not present in the water, so that future sampling schedules would be more cost effective. After identifying the major problem, work was concentrated on determining the extent of the problem and the magnitude of its effects on river quality from Calexico to the Salton Sea. Along with the concentration of selected organic constituents, DO concentration was considered the primary indicator of the health of the river. Measurements and determinations were made around the clock within a diel (24-hour) period to show the effects of respiration and photosynthesis on river quality as well as longitudinally by river mile in order to determine the effects of oxidation. A 
time-of-travel investigation was made to show the rate of migration of water from Calexico to the Salton Sea, and a composite sampling program was made at the Calexico gage to determine the loads of selected constituents. For the determination and identification of benthic invertebrate and phytoplankton communities as indicators of environmental conditions, biological samples were collected on the New River and at a control site on the Alamo River.

Major topics covered in the report are dissolved oxygen, organic material, and biota. The reconnaissance study, river profile study, diel study, time-of-travel investigation, and composite sampling were completed during the period 1977 to 1979 in cooperation with the California Regional Water Quality Control Board, Colorado River Basin Region. The biota sampling program was completed in 1978 and 1979 in cooperation with the California Department of Water Resources.

\section{Field and Laboratory Procedures}

Water samples for field measurement and for laboratory analysis were collected at the centroid of flow by using an open-mouth glass bottle when water velocities were less than $2.0 \mathrm{ft} / \mathrm{s}$. A depth-integrated suspended-sediment sampler was used when water velocities exceeded $2.0 \mathrm{ft} / \mathrm{s}$. At the Calexico gage, where the most intensive sampling occurred, a cross-sectional profile of the river showed a range in specific conductance of less than 1.5 percent at $7,000 \mu$ mho indicating a well-mixed water. For the river profile measured during the week of September 26-30, 1977, water samples were collected from a small boat traveling upstream from the Westmorland gage.

Samples for laboratory determination were sent to the U.S. Geological Survey's Central Laboratory at Arvada, Colo. Methods used for analysis of most constituents are those described by Skougstad and others (1979). Pesticides were determined by gas chromatography, organic carbon was determined by wet oxidation, oil and grease were determined by ether extraction, and radioactivity was determined by U.S. Geological Survey standard methods (Goerlitz and Brown, 1972; Thatcher and others, 1977). Biological samples were collected according to methods established by Greeson and others (1977).

Field determinations (specific conductance, temperature, dissolved oxygen, $\mathrm{pH}$, and turbidity) were made in accordance with standard U.S. Geological Survey procedures (Skougstad and others, 1979).

Field tests not conducted according to U.S. Geological Survey standard methods may be subject to unknown interferences. They should not be considered to be of the same accuracy and precision as those analyses done by the Survey laboratory. Field tests using color-wheel comparison have detection limits of $0.1 \mathrm{mg} / \mathrm{L}$ for chromium, copper, and manganese. These constituents, when determined by the Survey's central laboratory, have a detection limit of $0.01 \mathrm{mg} / \mathrm{L}$. Relative fluorescence was determined by a fluorometer.

\section{RECONNAISSANCE}

\section{Data Collection}

The reconnaissance study during the week of May 9,1977 , was a single intensive sampling of the New River at Calexico. Objectives of the sampling were twofold: (1) To characterize the water entering the United States from Mexico, and (2) to determine the properties and constituents that degrade river quality as a basis for developing a comprehensive study. Samples collected in May are considered to be fairly representative of the year.

A comprehensive list of deleterious materials that might be detected in the New River at Calexico was developed on the basis of constituents generally associated with industrial, commercial, and agricultural practices. The potential toxicity of the suspected constituents to wildlife in particular and to degradation of the river in general was considered in order to reduce the list to a workable size, in terms of the economics and logistics of sampling and analysis. The New River at Calexico is part of the National Stream Quality Accounting Network (NASQAN), a federally funded program involving a monthly and quarterly sampling of sites representative of selected hydrologic basins. The monthly schedule includes samples for the determination of common ions, nutrients, and some organic constituents. The quarterly schedule also has, in addition to the monthly schedule of constituents and characteristics, determinations for trace elements and selected organic constituents.

During the reconnaissance, field tests and measurements for the following selected constituents and characteristics were made on water samples collected at about 45-minute intervals (tables 1 and 2, figs. 5 and 6):

$\begin{array}{ll}\text { Specific conductance } & \text { Turbidity } \\ \mathrm{pH} & \text { Dissolved oxygen }\end{array}$

Water temperature

Together with the appearance of the river, these tests were used to signal the passage of unusually polluted water.

Samples for laboratory analysis of the following constituents were collected whenever the appearance of the river and the field tests indicated an increase in pollution:
Chemical oxygen demand

Total filtrable residue

Total nonfiltrable residue Antimony
Gross beta radiation Total organic carbon Dissolved organic carbon Cyanide 
Table 1. Field measurements of selected physical and chemical characteristics, New River at Calexico, May 9 13,1977

\begin{tabular}{|c|c|c|c|c|c|c|}
\hline DATE & TIME & $\begin{array}{l}\text { STREAM- } \\
\text { FLOW, } \\
\text { INSTAN- } \\
\text { TANEOUS } \\
\text { (CFS) }\end{array}$ & $\begin{array}{l}\text { SPE- } \\
\text { CIF IC } \\
\text { CON- } \\
\text { DUCT- } \\
\text { ANCE } \\
\text { (UMHOS) }\end{array}$ & $\begin{array}{c}\text { PH } \\
\text { (UNITS) }\end{array}$ & $\begin{array}{l}\text { TEMPER- } \\
\text { ATURE } \\
\text { (DEG C) }\end{array}$ & $\begin{array}{l}\text { TUR- } \\
\text { GID- } \\
17 Y \\
\text { (JTU) }\end{array}$ \\
\hline \multicolumn{7}{|l|}{ MAY } \\
\hline $09 \ldots$ & 1710 & 156 & 7500 & 7.7 & 23.0 & 35 \\
\hline $09 . .$. & 1830 & 153 & 7510 & 7.8 & 22.6 & 35 \\
\hline $09 \ldots$ & 1715 & 153 & 7540 & 7.6 & 22.3 & 36 \\
\hline $09 \ldots$ & 2000 & $15 y$ & 7560 & 7.7 & 22.0 & 34 \\
\hline $09 \ldots$ & 2045 & 154 & 7580 & 8.0 & 21.7 & 35 \\
\hline $09 \ldots$ & 2130 & 159 & 7600 & 7.7 & 21.5 & 36 \\
\hline $09 . \ldots$ & $<215$ & $15 y$ & 7600 & 7.7 & 21.3 & 37 \\
\hline $09 \ldots$ & 2300 & 15y & 7670 & 7.7 & 21.1 & 30 \\
\hline $04 \ldots$ & 2345 & 158 & 7670 & 7.6 & 21.0 & 26 \\
\hline $10 \ldots$ & 0030 & 158 & 7890 & 7.5 & 20.0 & 30 \\
\hline $10 \ldots$ & 0115 & 157 & 7960 & 7.6 & 20.4 & 30 \\
\hline $10 \ldots$ & 0200 & 155 & 8080 & 7.5 & 20.0 & 26 \\
\hline $10 \ldots$ & 0245 & 153 & 8130 & 8.2 & 19.6 & 28 \\
\hline $10 \ldots$ & 0330 & 15b & 8150 & 7.5 & 19.4 & 26 \\
\hline $10 \ldots$ & 0420 & $15 y$ & 8170 & 7.4 & 19.0 & 26 \\
\hline $10 \ldots$ & 0500 & $15 y$ & 8140 & 7.3 & 18.9 & 28 \\
\hline $10 \ldots$ & 0545 & 159 & 7940 & 7.4 & 18.6 & 31 \\
\hline $10 \ldots$ & 0630 & IS9 & 7600 & 7.3 & 18.4 & 32 \\
\hline $10 \ldots$ & 0715 & 131 & 7780 & 7.3 & 18.3 & 27 \\
\hline $10 \ldots$ & 0820 & 151 & 7650 & 7.5 & 18.4 & 22 \\
\hline $10 \ldots$ & 0910 & 1 1 & 7620 & 7.5 & 18.7 & 25 \\
\hline $10 \ldots$ & 1030 & 156 & 8040 & 7.6 & 19.5 & 32 \\
\hline $10 \ldots$ & 1115 & כ5 & 8160 & 7.6 & 20.0 & 32 \\
\hline $10 \ldots$ & 1200 & 155 & 8230 & 7.6 & 20.5 & 30 \\
\hline $10 \ldots$ & 1245 & לS15 & 8180 & 7.4 & 21.0 & 30 \\
\hline $10 \ldots$ & 1330 & 161 & 7700 & 7.4 & 21.6 & 30 \\
\hline $10 \ldots$ & 1415 & 107 & 8130 & 7.4 & 21.9 & 28 \\
\hline $10 \ldots$ & 1530 & $15 \theta$ & 8040 & 7.4 & 22.4 & 32 \\
\hline $10 \ldots$ & 1615 & 156 & 8010 & 7.6 & 22.5 & 36 \\
\hline $10 \ldots$ & 1700 & 153 & 8030 & 7.4 & 22.5 & 31 \\
\hline $10 \ldots$ & 1745 & 153 & 8080 & 7.6 & 22.5 & 39 \\
\hline $10 \ldots$ & 1830 & 161 & 8100 & 7.6 & 22.5 & 32 \\
\hline $10 \ldots$ & 2000 & 159 & 0190 & 7.6 & 22.2 & 30 \\
\hline $10 \ldots$ & 2045 & 154 & 8140 & 7.6 & 22.0 & 30 \\
\hline $10 \ldots$ & 2130 & 157 & 8100 & 7.6 & 21.4 & 25 \\
\hline $10 \ldots$ & 2215 & 154 & 0180 & 7.6 & 21.7 & 25 \\
\hline $10 \ldots$ & 2300 & 153 & 8200 & 7.5 & 21.5 & 26 \\
\hline $10 \ldots$ & 2345 & 152 & 8200 & 7.6 & 21.4 & 29 \\
\hline $11 \ldots$ & 0030 & 152 & 8220 & 7.4 & 21.1 & 24 \\
\hline $11 \ldots$ & 0115 & 151 & 8440 & 7.4 & 20.9 & 22 \\
\hline $11 \ldots$ & 0200 & 150 & 8410 & 7.4 & 20.7 & 20 \\
\hline $11 \ldots$ & 0300 & 148 & 8380 & 7.4 & 20.4 & 25 \\
\hline $11 \ldots$ & 0415 & 149 & 8400 & 7.4 & 20.0 & 30 \\
\hline $11 \ldots$ & 0500 & 150 & 8400 & 7.5 & 19.6 & 28 \\
\hline $11 \ldots$ & 0545 & 150 & 8350 & 7.4 & 19.4 & 21 \\
\hline $11 \ldots$ & 0030 & 149 & $0>00$ & 7.4 & 19.1 & 24 \\
\hline $11 \ldots$ & 0715 & 145 & 8500 & 7.6 & 19.0 & 17 \\
\hline $11 \ldots$ & 0015 & 144 & 0350 & 7.5 & 18.2 & 30 \\
\hline $11 \ldots$ & 0900 & 144 & d3s0 & 7.6 & 19.5 & 40 \\
\hline $11 \ldots$ & 0945 & 144 & 8200 & 7.7 & 19.4 & 40 \\
\hline $11 \ldots$ & 1030 & 144 & o150 & 7.6 & 20.4 & 36 \\
\hline $11 \ldots$ & 1115 & 144 & 0200 & 7.5 & 20.6 & 42 \\
\hline $11 \ldots$ & 1200 & 144 & 8050 & 7.6 & 21.4 & 43 \\
\hline $11 \ldots$ & 1245 & 144 & 8000 & 7.6 & 21.8 & 45 \\
\hline $11 \ldots$ & 1330 & 157 & 8000 & 7.6 & 22.2 & 45 \\
\hline $11 \ldots$ & 1415 & 147 & 8000 & 7.6 & 22.6 & 44 \\
\hline $11 \ldots$ & 1500 & 144 & 8000 & 7.6 & 22.9 & 43 \\
\hline $11 \ldots$ & 1545 & 144 & 8000 & 7.7 & 23.1 & $3 y$ \\
\hline $11 \ldots$ & 1030 & 151 & 8000 & 7.6 & 23.2 & 37 \\
\hline $11 \ldots$ & 1715 & 154 & Bluo & 7.7 & 23.2 & 33 \\
\hline $11 \ldots$ & 1800 & 141 & 0100 & 7.0 & 23.2 & 32 \\
\hline $11 \ldots$ & 1415 & 140 & bluo & 7.5 & 23.0 & 32 \\
\hline $11 \ldots$ & 2015 & 144 & 8100 & 7.6 & 22.8 & 24 \\
\hline $11 \ldots$ & 2120 & 144 & olso & 7.6 & 22.6 & 27 \\
\hline $11 \ldots$ & 2205 & 144 & 0150 & 7.5 & 22.4 & 27 \\
\hline $11 \ldots$ & $2 \angle 50$ & 155 & 8150 & 7.5 & 22.1 & 24 \\
\hline $11 \ldots$ & 2333 & iss & 8150 & 7.5 & 22.0 & 23 \\
\hline $12 \ldots$ & 0015 & 140 & 8200 & 7.5 & 21.8 & 36 \\
\hline $12 \ldots$ & 0100 & 146 & 8200 & 7.5 & 21.8 & 32 \\
\hline $12 \ldots$ & 0145 & 146 & 8200 & 7.7 & 21.6 & 30 \\
\hline $12 \ldots$ & 0230 & 144 & 8300 & 7.6 & 21.6 & 27 \\
\hline $12 \ldots$ & 0315 & 144 & 8200 & 7.6 & 21.1 & 30 \\
\hline $12 .$. & 0415 & 144 & 8400 & 7.7 & 20.7 & 36 \\
\hline $12 \ldots$ & 0500 & 144 & 8300 & 7.7 & 20.3 & 32 \\
\hline $12 \ldots$ & 0545 & 144 & 0200 & 7.6 & 20.0 & 36 \\
\hline $12 \ldots$ & 0630 & 144 & 0300 & 7.7 & 19.8 & 33 \\
\hline $12 \ldots$ & 0715 & 140 & 8200 & 7.7 & 19.7 & 33 \\
\hline $12 \ldots$ & 0800 & 137 & 8500 & 7.6 & 19.6 & 23 \\
\hline
\end{tabular}

Table 1. Field measurements of selected physical and chemical characteristics, New River at Calexico, May 9 13, 1977-Continued

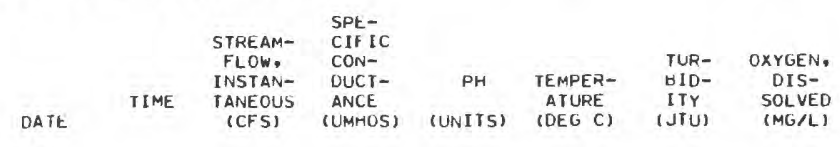

\begin{tabular}{|c|c|c|c|}
\hline \multicolumn{4}{|l|}{ MAY } \\
\hline $12 \ldots$ & 6850 & 137 & 8400 \\
\hline $12 \ldots$ & 0905 & 137 & 8400 \\
\hline $12 \ldots$ & 0920 & 137 & 8400 \\
\hline $12 \ldots$ & 0922 & 131 & 8400 \\
\hline $12 \ldots$ & 0927 & 136 & 8450 \\
\hline $12 . .$. & 0930 & 130 & 8450 \\
\hline $12 \ldots$ & 0932 & 130 & 8450 \\
\hline $12 \ldots$ & 0937 & 136 & 8450 \\
\hline $12 \ldots$ & 0942 & 136 & 8400 \\
\hline $12 \ldots$ & 0950 & 136 & 8250 \\
\hline $12 \ldots$ & 0955 & 136 & 8200 \\
\hline $12 \ldots$ & 1000 & 136 & 8200 \\
\hline $12 .$. & 1002 & 136 & 8200 \\
\hline $12 \ldots$ & 1008 & 135 & 8200 \\
\hline $12 \ldots$ & 1105 & 136 & 8100 \\
\hline $12 .$. & 1340 & 146 & 8000 \\
\hline $12 \ldots$ & 1445 & 146 & 8000 \\
\hline $12 \ldots$ & 1315 & 150 & 8050 \\
\hline $12 \ldots$ & 1545 & 143 & 8000 \\
\hline $12 .$. & 1615 & 143 & 8000 \\
\hline $12 \ldots$ & 1645 & 145 & 8000 \\
\hline $12 \ldots$ & 1715 & 145 & 8050 \\
\hline $12 \ldots$ & 1815 & 146 & 8000 \\
\hline $12 \ldots$ & 1830 & 146 & 8000 \\
\hline $12 \ldots$ & 1845 & 146 & 8000 \\
\hline $12 \ldots$ & 1915 & 146 & 8050 \\
\hline $12 .$. & 2000 & 147 & 8100 \\
\hline $12 \ldots$ & 2030 & 147 & 8000 \\
\hline $12 \ldots$ & 2100 & 143 & 8000 \\
\hline $12 \ldots$ & 2130 & 143 & 8000 \\
\hline $12 \ldots$ & 2200 & 143 & 8000 \\
\hline $12 \ldots$ & 2230 & 143 & 8000 \\
\hline $12 \ldots$ & 2300 & 143 & 8000 \\
\hline $12 \ldots$ & 2330 & 143 & 8000 \\
\hline $12 \ldots$ & 2345 & 141 & 8000 \\
\hline $12 .$. & 2400 & 141 & 8300 \\
\hline $13 . .$. & 0030 & 141 & 8400 \\
\hline $13 \ldots$ & 0100 & 147 & 8500 \\
\hline $13 \ldots$ & 0130 & 147 & 8500 \\
\hline $13 \ldots$ & 0200 & 146 & 8500 \\
\hline $13 \ldots$ & 0230 & 146 & 8550 \\
\hline $13 \ldots$ & 0300 & 145 & 8500 \\
\hline $13 . .$. & 0345 & 146 & 8500 \\
\hline $13 . .$. & 0430 & 146 & 8500 \\
\hline $13 \ldots$ & 0515 & 147 & 0450 \\
\hline $13 \ldots$ & 0600 & $14 b$ & 8400 \\
\hline $13 .$. & 0645 & 145 & 8250 \\
\hline 13. & 0730 & 145 & $8<00$ \\
\hline 13. & 0015 & 130 & 8200 \\
\hline $13 .$. & $0 \ngtr 00$ & 132 & 8000 \\
\hline $13 \ldots$ & 1030 & 132 & 7650 \\
\hline
\end{tabular}

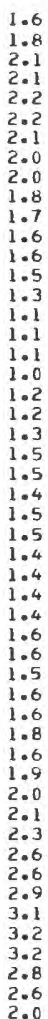

Table 2. Summary of water-quality field data, New River at Calexico, May 9-13, 1977

[Mean based on 129 observations]

\begin{tabular}{|c|c|c|c|}
\hline Constituent & $\begin{array}{l}\text { Range in } \\
\text { concentration } \\
\text { or value }\end{array}$ & Mean & $\begin{array}{l}\text { Standard } \\
\text { deviation }\end{array}$ \\
\hline Specific conductance--mmho-- & $7,500-8,500$ & 8,300 & 243 \\
\hline pH---1-- units-- & $7.3-8.8$ & -- & -- \\
\hline Water temperature---- $-{ }^{\circ} \mathrm{C}--$ & $17.8-23.2$ & 20.4 & 1.4 \\
\hline Turbidity---- & $20-115$ & 37 & 17.0 \\
\hline Dissolved oxygen------mg/L-- & $0.3-3.2$ & 1.7 & 0.6 \\
\hline
\end{tabular}

Barium

Chromium

Copper

Lead

Mercury

Nickel

Gross alpha radiation
Phenol

Methylene blue active substances

Oil and grease

Tannin and lignin

Organochlorine insecticides

Organophosphorus insecticides

\section{Results}

On May 12, 1977, at 0905 hours, the first major pollutant slug of the reconnaissance occurred. It was first observed as white particulate matter and confirmed by an increase in turbidity and $\mathrm{pH}$ (table 1 and fig. 5). The slug of pollution apparently peaked around 0935 at the Calexico gage, then receded as indicated by the gradual 


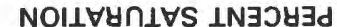

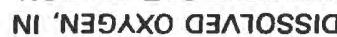

요

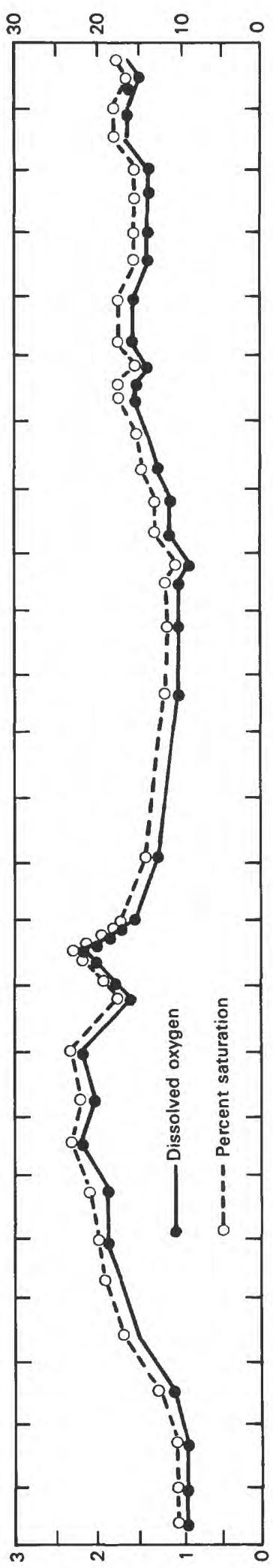

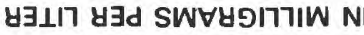

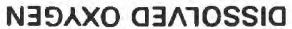

Y $\exists \perp \exists W I \perp N \exists J$ Y 9 d SOHWOYJIW NI

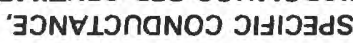
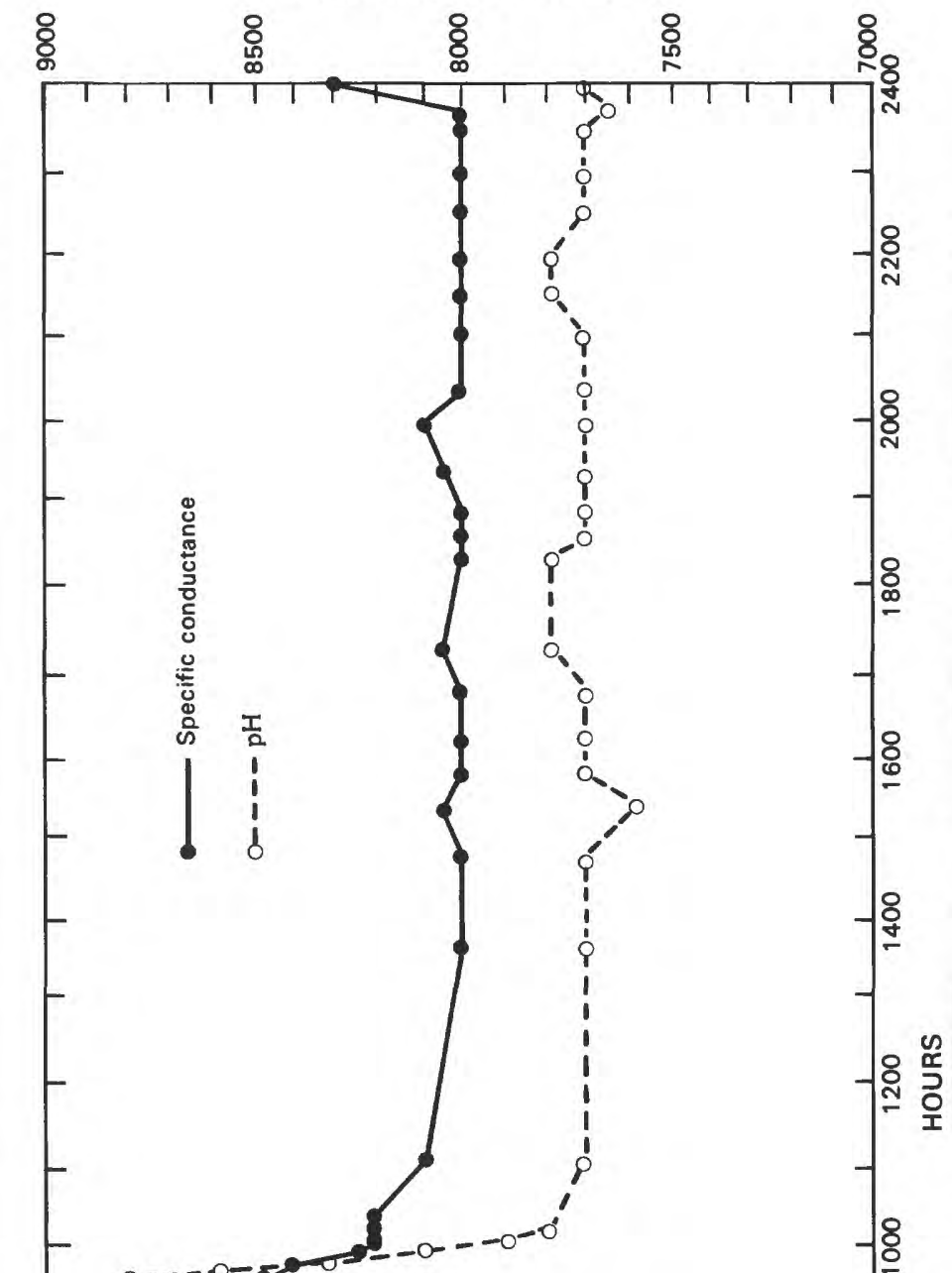

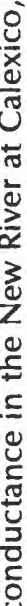

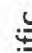

¿

돌

İ

要

우

党

¿ัญ
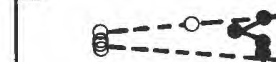

$-$

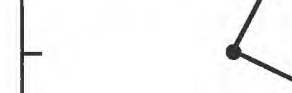

$-$

$-$

$-$

$-$

$-$

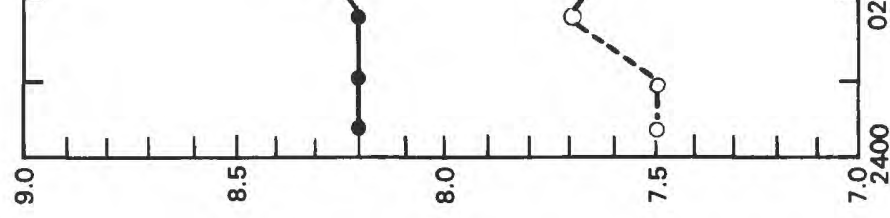

$\mathrm{H}^{\mathrm{d}}$ 


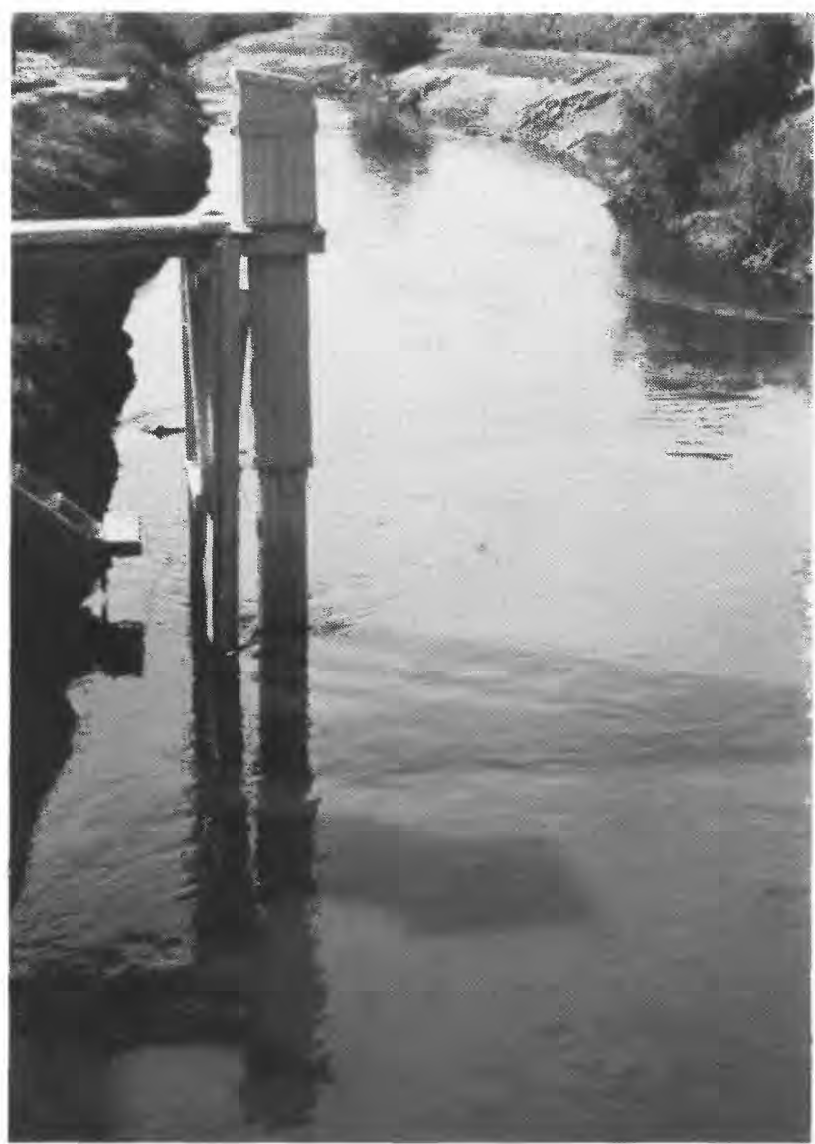

Figure 6. New River at Calexico gaging station. View is downstream.

decrease in turbidity and $\mathrm{pH}$ to background levels. Turbidity (table 1) and concentrations of total organic carbon (TOC)(table 3) indicated that additional pollutant slugs passed the gage May 12. Samples were collected at 1545 , 1715 , and 2130 hours when turbidity rose above background levels. In all, 14 sets of samples were collected. In addition, a set of control samples was collected from the Alamo River at Drop 9 near Holtville, where the discharge is about equal to that of the New River at Calexico but consists primarily of agricultural return water (table 4).

Because of the industry present in Mexicali, it was anticipated that trace elements would be found in the New River at Calexico. None, however, was observed in concentrations indicative of industrial sources (table 3). Of the trace elements, barium had the highest concentration, $200 \mu \mathrm{g} / \mathrm{L}$. The limit for domestic water supplies is 1,000 $\mu \mathrm{g} / \mathrm{L}$ (Environmental Protection Agency, 1976). Nor is $200 \mu \mathrm{g} / \mathrm{L}$ of barium indicative of major industrial pollution.

Small amounts of various pesticides were detected during the reconnaissance; the highest concentration was $1.6 \mu \mathrm{g} / \mathrm{L}$ of malathion at 1415 hours on May 10. The arithmetic mean malathion concentration for the 14 sam- ples collected during the reconnaissance was $0.27 \mu \mathrm{g} / \mathrm{L}$. To protect the integrity of freshwater aquatic life, the U.S. Environmental Protection Agency (EPA)(1976) has established a criterion concentration of $0.1 \mu \mathrm{g} / \mathrm{L}$, which was exceeded by 5 of the 14 samples. DDT concentrations ranging from 0.00 to $0.25 \mu \mathrm{g} / \mathrm{L}$ were detected in the New River at Calexico along with its breakdown products DDD and DDE. To protect aquatic life, EPA has established a criterion concentration of $0.001 \mu \mathrm{g} / \mathrm{L}$ of DDT in freshwater. The arithmetic mean DDT concentration was 0.08 $\mu \mathrm{g} / \mathrm{L}$ for the 13 samples collected during the reconnaissance. Concentrations of DDT in all but one of these samples exceeded the criterion. Even though use of DDT is banned in the United States, low concentrations are still being found because of its persistence in the environment. It is not surprising, therefore, that concentrations of those pesticides were detected in the New River at Calexico. For lindane, the EPA criterion concentration to protect freshwater aquatic life is $0.01 \mu \mathrm{g} / \mathrm{L}$. The arithmetic mean concentration was $0.14 \mu \mathrm{g} / \mathrm{L}$, with concentrations of only two of the samples exceeding the criterion. Other compounds whose concentrations exceeded the EPA criteria were: phenols, arithmetic mean concentration of $12 \mu \mathrm{g} / \mathrm{L}$ and a criterion concentration of $1 \mu \mathrm{g} / \mathrm{L}$, which was exceeded by all the samples; cyanide, arithmetic mean concentration of $0.008 \mathrm{mg} / \mathrm{L}$ and a criterion concentration of $0.005 \mathrm{mg} / \mathrm{L}$, which was exceeded by 10 of the $14 \mathrm{sam}-$ ples; and diazinon, arithmetic mean concentration of $0.074 \mu \mathrm{g} / \mathrm{L}$ and a criterion concentration of $0.009 \mu \mathrm{g} / \mathrm{L}$, which was exceeded by all the samples.

\section{Comparisons}

Table 5 presents comparative data for samples collected from the Calexico gage during periods of little or no visible pollution (background) and periods of obvious pollution (slug), and from the control site on the Alamo River (control). Although the specific conductance for the New River at Calexico is more than two times that of the control site, with the exception of chemical oxygen demand (COD), phenols, and TOC, constituent values at the Calexico gage compare favorably with those of the Alamo River. Values detected during slug flows indicate the extent of the organic-pollution problem in the New River at Calexico.

In summary, the results from the reconnaissance trip indicate that the main problem in the New River at Calexico is organic pollution. With COD's of $510 \mathrm{mg} / \mathrm{L}$ and TOC's exceeding $160 \mathrm{mg} / \mathrm{L}$, it is apparent that the slug discharges severely stress the oxidative processes of the water crossing the boundary. It is also apparent, however, that flows having only background levels of organic material provide sufficient oxygen demand to degrade the river for many miles. Although no detailed water-quality studies involving COD, TOC, and DOC (dissolved organic carbon) have been made of the Alamo River, no 
Table 3. Laboratory analyses of water, New River at Calexico, May 10-12, 1977

\begin{tabular}{|c|c|c|c|c|c|c|c|c|c|c|c|c|}
\hline UATE & TIME & $\begin{array}{l}\text { OXYGEN } \\
\text { DEMANO, } \\
\text { CHEM- } \\
\text { ICAL } \\
\text { (HIGH } \\
\text { LEVEL) } \\
\text { (MG/L) }\end{array}$ & $\begin{array}{l}\text { SOL IDS, } \\
\text { RESIDUE } \\
\text { AT } 105 \\
\text { DEG. C. } \\
\text { DIS- } \\
\text { SOLVED } \\
(M G / L)\end{array}$ & $\begin{array}{l}\text { SOLIOS, } \\
\text { RESIDUE } \\
\text { AT } 105 \\
\text { DEG. C, } \\
\text { SUS- } \\
\text { PENDED } \\
\text { (MG/L) }\end{array}$ & $\begin{array}{l}\text { ANTI- } \\
\text { MONY, } \\
\text { TOTAL } \\
\text { (UL/L } \\
\text { AS SB) }\end{array}$ & $\begin{array}{l}\text { BARIUM, } \\
\text { TOTAL } \\
\text { RECOV- } \\
\text { ERABLE } \\
\text { (UG/L } \\
\text { AS BA) }\end{array}$ & $\begin{array}{l}\text { CHRO- } \\
\text { MIUM, } \\
\text { TOTAL } \\
\text { RECOV- } \\
\text { ERABLE } \\
\text { (UG/L } \\
\text { AS CR) }\end{array}$ & $\begin{array}{l}\text { COPPER, } \\
\text { TOTAL } \\
\text { RECOV- } \\
\text { ERABLE } \\
\text { (UG/L } \\
\text { AS CUI }\end{array}$ & $\begin{array}{l}\text { LEAD. } \\
\text { TOTAL } \\
\text { RECOV- } \\
\text { ERABLE } \\
\text { (UG/L } \\
\text { AS PB) }\end{array}$ & $\begin{array}{l}\text { MERCURY } \\
\text { TOTAL } \\
\text { RECOV- } \\
\text { ERABLE } \\
\text { (UG/L } \\
\text { AS HG) }\end{array}$ & $\begin{array}{l}\text { NICKEL, } \\
\text { TOTAL } \\
\text { RECOV- } \\
\text { ERABLE } \\
\text { (UG/L } \\
\text { AS NI) }\end{array}$ & $\begin{array}{l}\text { GKOSS } \\
\text { ALPHA, } \\
\text { UIS- } \\
\text { SOLVEU } \\
\text { (UG/L } \\
\text { AS } \\
\text { U-NAT) }\end{array}$ \\
\hline \multicolumn{13}{|l|}{ MAY } \\
\hline $10 \ldots$ & 0910 & 330 & 5600 & 32 & 2 & 200 & $<20$ & 20 & $<200$ & $<.5$ & $<50$ & $<69$ \\
\hline $10 \ldots$ & 1415 & 340 & 5700 & 48 & 2 & 200 & $<20$ & 80 & $<200$ & $<.5$ & 50 & 71 \\
\hline $11 \ldots$ & 0300 & 390 & 6100 & 35 & $<1$ & 200 & $<20$ & 20 & $<200$ & $<.5$ & 50 & 90 \\
\hline $11 \ldots$ & 1800 & 370 & - & -- & 2 & 200 & ND & 30 & $<200$ & $<.5$ & 50 & -- \\
\hline $12 \ldots$ & 0800 & 190 & 6100 & 110 & $<1$ & 200 & $<20$ & 20 & $<200$ & $<.5$ & $<50$ & $<71$ \\
\hline $12 \ldots$ & 0850 & 190 & - & -- & 2 & 200 & ND & 20 & $<200$ & $<.5$ & 50 & $=-$ \\
\hline $12 \ldots$ & 0905 & 400 & - & -- & 1 & 200 & $<20$ & 20 & $<200$ & $<.5$ & $<200$ & $\cdots$ \\
\hline $12 \ldots$ & 0920 & 510 & 5900 & 170 & 2 & 200 & $<<0$ & 20 & $<200$ & $<.5$ & 50 & $<37$ \\
\hline $12 \ldots$ & 0930 & -- & - & -- & 2 & 200 & 20 & 30 & $<200$ & $<.5$ & 50 & -- \\
\hline $1<\ldots$ & 0950 & 290 & 5800 & 72 & $<1$ & 200 & $<20$ & 20 & $<200$ & $<.5$ & 50 & $<81$ \\
\hline $12 \ldots$ & 1000 & 300 & 6000 & 50 & -- & 200 & $<20$ & 20 & $<200$ & $<.5$ & $<50$ & $<73$ \\
\hline $12 \ldots$ & $154 \mathrm{~S}$ & 330 & 5700 & 38 & $<1$ & 200 & $<20$ & 20 & $<200$ & $<.5$ & 50 & 65 \\
\hline $12 \ldots$ & 1715 & 350 & 6000 & 52 & -- & 200 & $<20$ & 40 & $<200$ & $<.5$ & 50 & $<36$ \\
\hline \multirow[t]{7}{*}{$12 \ldots$} & 2130 & 370 & 0100 & 50 & 1 & 200 & $<20$ & 30 & $<200$ & $<.5$ & 50 & 73 \\
\hline & GROSS & GROSS & GROSS & GROSS & GROSS & & & & & ME THY - & & \\
\hline & ALPHA, & BETA, & BETA. & BETA, & BETA. & & CARBON, & & & LENE & & \\
\hline & SUSP. & OIS- & SUSP. & OIS- & SUSP. & CARBON & ORGANIC & & & BLUE & & \\
\hline & TOTAL & SOLVED & TOTAL & SOLVED & TOTAL & ORGANIC & DIS- & CYANIDE & & ACTIVE & OIL & TANNIN \\
\hline & CUG/L & $(P C 1 / L$ & $(P C I / L$ & $1 P C I / L$ & (PCI/L & TOTAL & SOLVEU & TOTAL & PHENOLS & SUB- & AND & AND \\
\hline & AS & AS & AS & AS SR/ & AS SR/ & IMG/L & IMG $/ L$ & $1 M G / L$ & & STANCE & GREASE & LIGNIN \\
\hline DATE. & U-NATI & $C S-1371$ & CS -137$)$ & $Y T-90)$ & $Y T-90)$ & AS CI & AS CI & AS $(N)$ & $(U G / L)$ & $(M G / L)$ & $(M G / L)$ & $(M G / L)$ \\
\hline
\end{tabular}

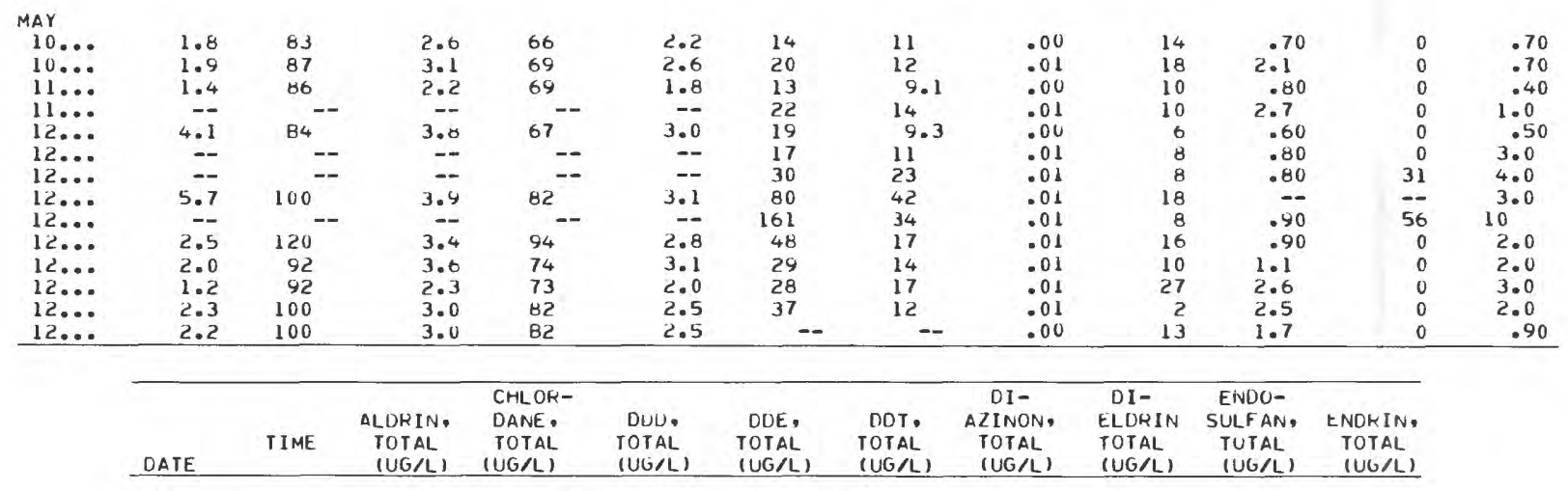

\begin{tabular}{|c|c|c|c|c|c|c|c|c|c|c|}
\hline \multicolumn{11}{|l|}{ MAY } \\
\hline $10 \ldots$ & $0 צ 10$ & .00 & .00 & .01 & .00 & .01 & .02 & .01 & .00 & .00 \\
\hline $10 \ldots$ & 1415 & -- & -- & -- & -- & -- & .20 & -- & -- & - \\
\hline $11 \ldots$ & 0300 & .00 & .00 & .01 & .00 & .01 & .01 & .00 & .00 & .00 \\
\hline $11 \ldots$ & 1800 & .00 & .00 & .04 & .01 & .08 & $.1<$ & .02 & .00 & .00 \\
\hline $12 . .$. & 0800 & .00 & .00 & .00 & .00 & .00 & .02 & .00 & .00 & .00 \\
\hline $12 \ldots$ & 0650 & .00 & .00 & .02 & .00 & .02 & .08 & .02 & .00 & .00 \\
\hline $12 \ldots$ & 0905 & $.0 u$ & .00 & .06 & .05 & .15 & לס. & .02 & .00 & .00 \\
\hline $12 \ldots$ & 0 ४บว० & .00 & .00 & .05 & .04 & .06 & .03 & .01 & .00 & .00 \\
\hline $12 \ldots$ & $0 ษ 30$ & .00 & .00 & .06 & .06 & .11 & .03 & .00 & .00 & .00 \\
\hline $12 \ldots$ & 0950 & .00 & .00 & .02 & .01 & .06 & .03 & .01 & .00 & .00 \\
\hline $12 \ldots$ & 1000 & .00 & .00 & .04 & .01 & .25 & .05 & .01 & .00 & .00 \\
\hline $12 \ldots$ & 1545 & .00 & .00 & .05 & $.0 ?$ & .14 & .11 & .02 & .00 & .00 \\
\hline $12 \ldots$ & 1715 & .00 & .00 & .09 & .03 & .13 & .14 & .02 & .00 & .00 \\
\hline $12 \ldots$ & 2130 & .00 & .00 & .04 & .02 & .05 & .14 & .01 & .00 & .00 \\
\hline
\end{tabular}

\begin{tabular}{|c|c|c|c|c|c|c|c|c|c|}
\hline DATE & $\begin{array}{l}\text { ETHION, } \\
\text { TOTAL } \\
\text { (UG/L) }\end{array}$ & $\begin{array}{l}\text { HEPTA- } \\
\text { CHLOR, } \\
\text { TUTAL } \\
\text { (UG/L) }\end{array}$ & $\begin{array}{l}\text { HEPIA- } \\
\text { CHLOR } \\
\text { EPOXIDE } \\
\text { TOTAL } \\
\text { (UG/LI }\end{array}$ & $\begin{array}{l}\text { LINUANE } \\
\text { TOTAL } \\
\text { (UG/L) }\end{array}$ & $\begin{array}{l}\text { MALA- } \\
\text { THION, } \\
\text { TOTAL } \\
\text { (UG/L) }\end{array}$ & $\begin{array}{l}\text { PAKA- } \\
\text { THION, } \\
\text { TOTAL } \\
\text { (UG/L) }\end{array}$ & $\begin{array}{l}\text { IUX- } \\
\text { APHENE, } \\
\text { TUIAL } \\
(U \cup / L)\end{array}$ & $\begin{array}{l}\text { TOTAL } \\
\text { TRI- } \\
\text { THION } \\
\text { (UG/L) }\end{array}$ & $\begin{array}{l}\text { NAPH- } \\
\text { THA- } \\
\text { LENES } \\
\text { POLY- } \\
\text { CHLUR. } \\
\text { TOTAL } \\
\text { (UG/L) }\end{array}$ \\
\hline \multicolumn{10}{|l|}{ MAY } \\
\hline $10 \ldots$ & .00 & .00 & .01 & .01 & .02 & .00 & 0 & .00 & .60 \\
\hline $10 \ldots$ & .00 & -- & -- & -- & 1.6 & .00 & -- & .00 & - \\
\hline $11 \ldots$ & .00 & .00 & .00 & .00 & .04 & .00 & 0 & .00 & - \\
\hline $11 \ldots$ & .00 & .00 & .00 & .00 & .36 & .00 & 0 & .00 & .00 \\
\hline $12 \ldots$ & .00 & .00 & .00 & .00 & .04 & .00 & 0 & .00 & .00 \\
\hline $12 \ldots$ & .00 & .00 & .00 & .00 & .05 & .00 & 0 & .00 & .00 \\
\hline $12 \ldots$ & .00 & .00 & .00 & .06 & .00 & .00 & 0 & .00 & .00 \\
\hline $12 \ldots$ & .00 & .00 & .00 & .02 & .00 & .00 & 0 & .00 & .00 \\
\hline $12 \ldots$ & .00 & .00 & .00 & .00 & .00 & .00 & 0 & .00 & - \\
\hline $12 \ldots$ & .00 & .00 & .00 & .00 & .04 & .00 & 0 & .00 & .00 \\
\hline $12 \ldots$ & .00 & .00 & .00 & .00 & .09 & .00 & 0 & .00 & .00 \\
\hline $12 \ldots$ & .00 & .00 & .00 & .00 & $.2 y$ & .00 & 0 & .00 & - \\
\hline $12 \ldots$ & .00 & .00 & .01 & .00 & .32 & .00 & 0 & .00 & .00 \\
\hline $1<\ldots$ & .00 & .00 & .00 & .00 & .90 & .00 & 0 & .00 & $=$ \\
\hline
\end{tabular}


Table 4. Field measurements and laboratory analyses of water at control site, Alamo River at Drop 9 near Holtville, May 12, 1977

\begin{tabular}{|c|c|}
\hline Constituent & $\begin{array}{l}\text { Concentration } \\
\text { or value }\end{array}$ \\
\hline Specific conductance-- & 3,560 \\
\hline 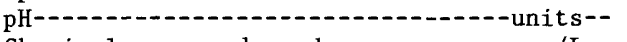 & 7.4 \\
\hline Chemical oxygen demand-- & 72 \\
\hline Total filtrable residue------- & 2,700 \\
\hline Nonfiltrable residue--- & 170 \\
\hline Antimony-- & 0 \\
\hline Barium------- & 100 \\
\hline Chromium---.-- & 10 \\
\hline Copper---:-- & 10 \\
\hline Lead-- - - & 100 \\
\hline 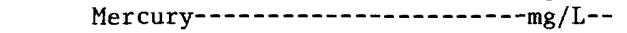 & 0.0 \\
\hline 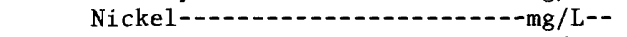 & 50 \\
\hline Dissolved gross alpha as U-nat-----mg/L-- & 60 \\
\hline Suspended gross alpha as U-nat------mg/L-- & 12 \\
\hline Dissolved gross beta as CS-137----pCi/L-- & 17 \\
\hline Suspended gross beta as CS-137----pCi/L-- & 8.4 \\
\hline Dissolved gross beta as SR90/Y90---pCi/L-- & 13 \\
\hline Suspended gross beta as SR90/Y90---pCi/L-- & 6.9 \\
\hline Total organic carbon- & 11 \\
\hline Dissolved organic carbon--- & 15 \\
\hline 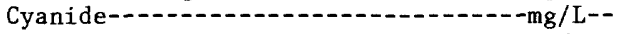 & 0.00 \\
\hline Phenols--1s & 2 \\
\hline Methylene blue active substances----mg/L-- & 0.20 \\
\hline Oil and grease-- & 0 \\
\hline Tannin and lignin-- & 0.9 \\
\hline Total aldrin- & 0.00 \\
\hline Chlordane-- & 0.0 \\
\hline 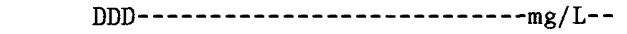 & 0.00 \\
\hline DDE-- & 0.02 \\
\hline DDT-1- & 0.01 \\
\hline 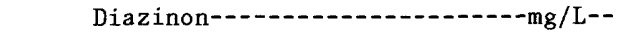 & 0.33 \\
\hline 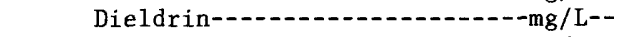 & 0.01 \\
\hline Endosulfan-- & 0.00 \\
\hline 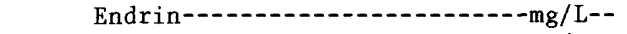 & 0.00 \\
\hline Ethion---1-- & 0.00 \\
\hline Heptachlor---1- & 0.00 \\
\hline Heptachlor epoxide---------mg/L-- & 0.00 \\
\hline Lindane-- & 0.00 \\
\hline 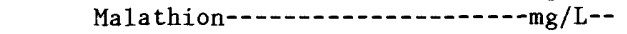 & 0.00 \\
\hline Parathion---1-1- & 0.00 \\
\hline Toxaphene-- & 0 \\
\hline 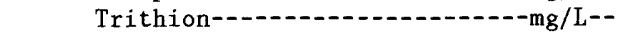 & 0.00 \\
\hline Polychlorinated naphthalenes------mg/L-- & 0.00 \\
\hline
\end{tabular}

Table 5. Comparison of water-quality constituents during periods of background and slug-flow conditions

[Control site on Alamo River]

\begin{tabular}{|c|c|c|c|}
\hline \multirow[t]{2}{*}{ Constituent } & \multicolumn{2}{|c|}{$\begin{array}{l}\text { Arithmetic mean } \\
\text { concentration } \\
\text { or value }\end{array}$} & \multirow[t]{2}{*}{ Control } \\
\hline & Background & Slug & \\
\hline Specific conductance-------mho-- & 8,300 & 8,300 & 3,560 \\
\hline 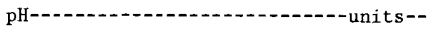 & $7.4-7.8$ & 8.8 & 7.4 \\
\hline Turbidity-- & $17-45$ & 115 & -- \\
\hline Total nonfilterable residue-----mg/L-- & 37 & 77 & 170 \\
\hline Chemical oxygen demand-------mg/L-- & 190 & 325 & 72 \\
\hline Total organic carbon---------mg/L-- & 17 & 50 & 11 \\
\hline Dissolved organic carbon------mg/L-- & 11 & 20 & 15 \\
\hline $0 \mathrm{il}$ and grease---- & 0 & 44 & 0 \\
\hline 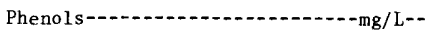 & 14 & 12 & 2 \\
\hline 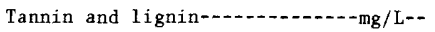 & 0.7 & 3 & 0.9 \\
\hline
\end{tabular}

such extremes in organic concentration have been observed on casual inspection by the U.S. Geological Survey or its cooperators.

\section{WATER-QUALITY INVESTIGATIONS}

\section{Pesticides and Trace Elements}

Although concentrations of some pesticides exceeded the EPA criteria in the 1977 reconnaissance, they were not considered to be a major problem when compared to the organic pollution. A detailed sampling three times a year was made 2 years later to determine if the situation had changed. The samples indicated that there was still no significant problem (table 6) with pesticides or trace elements.

\section{Dissolved Oxygen}

Dissolved oxygen is a major indicator of the ongoing physical, chemical, and biological processes occurring in the aquatic environment and therefore provides an index of the health of the system. Because DO is an excellent indicator and because it is easily measured in the field, emphasis was placed on obtaining DO information at selected sites on the New River.

The factors regulating DO concentration are reaeration, turbulence, temperature, photosynthesis, respiration, oxidation, atmospheric pressure, and tributary inflow. Only reaeration, turbulence, photosynthesis, and tributary inflow are potential contributors of oxygen, and only reaeration provides a continual replenishment. The amount of oxygen released by photosynthesis is dependent upon light intensity, turbidity of the water, and the density of the periphyton, phytoplankton, and submerged plant communities. Net oxygen production occurs when photosynthetic rates exceed respiration rates. The factors affecting photosynthesis, however, can be unfavorable, and no net production of oxygen will result. Tributary inflow also works in two ways. Inflow high in DO concentration will enhance the health of a stream if that stream has oxygen depletion, or, conversely, the health of a stream is impaired if the inflow is low in DO concentration.

In reaeration, oxygen enters the water at the airwater interface as an infinitely thin film that is instantly saturated (Velz, 1970, p. 189). Subsurface diffusion is then responsible for spreading the dissolved oxygen. Turbulence greatly increases the rate at which saturated surface layers are mixed with the unsaturated water and also decreases the depth through which the oxygen must diffuse. In general, the rate of reaeration is proportional to the difference between the observed DO concentration and the DO concentration at saturation, although the actual amount received depends on physical and hydrologic 
Table 6. Chemical and physical analyses of water, 1978-79, at selected sites

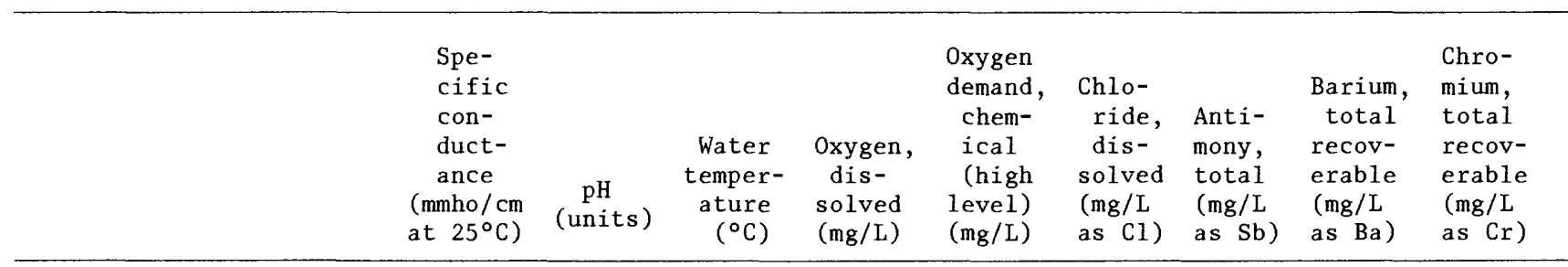

NEW RIVER AT INTERNATIONAL BOUNDARY, AT CALEXICO

\begin{tabular}{|c|c|c|c|c|c|c|c|c|c|}
\hline Number of observations & 3 & 3 & 3 & 3 & 3 & 3 & 3 & 3 & 3 \\
\hline Arithmetic mean & 6420 & -- & 24.5 & 0.7 & 140 & 1870 & 2 & 200 & 1 \\
\hline EPA criteria & -- & $6.5-9.0$ & (1) & 5.0 & - & - & -- & -- & 100 \\
\hline Number of times exceeded & -- & 0 & - & 3 & -- & -- & -- & -- & 0 \\
\hline
\end{tabular}

NEW RIVER AT LYONS CROSSING

\begin{tabular}{|c|c|c|c|c|c|c|c|c|c|}
\hline Number of observations & 3 & 3 & 3 & 3 & 3 & 2 & 2 & 3 & 3 \\
\hline Arithmetic mean & 6670 & -- & 26.3 & 0.3 & 95 & 2200 & 2 & 130 & 8.3 \\
\hline EPA criteria & -- & $6.5-9.0$ & (1) & 5.0 & -- & -- & -- & -- & 100 \\
\hline Number of times exceeded & -- & 0 & -- & 3 & -- & -- & -- & -- & 0 \\
\hline
\end{tabular}

NEW RIVER AT KEYSTONE

\begin{tabular}{|c|c|c|c|c|c|c|c|c|c|}
\hline Number of observations & 2 & 2 & 2 & 2 & 2 & 1 & 2 & 2 & 2 \\
\hline Arithmetic mean & 5500 & -- & 26.1 & 4.4 & 60 & 1700 & 1 & 150 & 0.5 \\
\hline EPA criteria & -- & $6.5-9.0$ & (1) & 5.0 & -- & -- & -- & -- & 100 \\
\hline Number of times exceeded & -- & 0 & - & 1 & -- & -- & -- & -- & 0 \\
\hline
\end{tabular}

NEW RIVER AT OUTLET NEAR WESTMORLAND

\begin{tabular}{|c|c|c|c|c|c|c|c|c|c|}
\hline Number of observations & 3 & 3 & 3 & 3 & 3 & 2 & 3 & 3 & 3 \\
\hline Arithmetic mean & 4780 & -- & 23.7 & 5.7 & 53 & 1100 & 1.3 & 170 & 7 \\
\hline EPA criteria & -- & $6.5-9.0$ & (1) & 5.0 & -- & -- & -- & -- & 100 \\
\hline Number of times exceeded & -- & 0 & -- & 0 & -- & -- & -- & -- & 0 \\
\hline
\end{tabular}

ALAMO RIVER AT OUTLET NEAR NILAND

\begin{tabular}{|c|c|c|c|c|c|c|c|c|c|}
\hline Number of observations & 3 & 3 & 3 & 3 & 3 & 2 & 3 & 3 & 3 \\
\hline Arithmetic mean & 3760 & -- & 23.7 & 7.5 & 42 & 705 & 1 & 170 & 2.3 \\
\hline EPA criteria & -- & $6.5-9.0$ & (1) & 5.0 & -- & -- & -- & -- & 100 \\
\hline Number of times exceeded & -- & 0 & -- & 0 & -- & -- & -- & -- & 0 \\
\hline
\end{tabular}

See footnotes at end of table.

characteristics such as temperature, water depth, occupied channel volume, and stream turbulence of each stretch of the river (Velz, 1970, p. 184). If the DO concentration in the river is zero, then the rate of reaeration is at its maximum.

\section{Temperature Effects}

Temperature plays a vital role in regulating the amount of oxygen available both to the biota of the stream and to the oxidative processes. For example, summer water temperature in the Imperial Valley exceeds $30^{\circ} \mathrm{C}$. The amount of oxygen that the water holds decreases as the temperature increases. Water at $20^{\circ} \mathrm{C}$ and 50 -percent saturation has a DO concentration of $4.5 \mathrm{mg} / \mathrm{L}$. Water at $34^{\circ} \mathrm{C}$ and 50 -percent saturation has a DO concentration of $3.5 \mathrm{mg} / \mathrm{L}$. Although a difference of $1.0 \mathrm{mg} / \mathrm{L}$ may seem small, it nevertheless represents a 20 -percent decrease in the amount of oxygen available for the aerobic oxidation of organic matter.

\section{Sag Curves (Profiles)}

As the aquatic system is stressed-for example, by heavy organic loads - that stress is reflected by changes in the DO concentration. By measuring the DO concentra- 
Table 6. Chemical and physical analyses of water, 1978-79, at selected sites-Continued

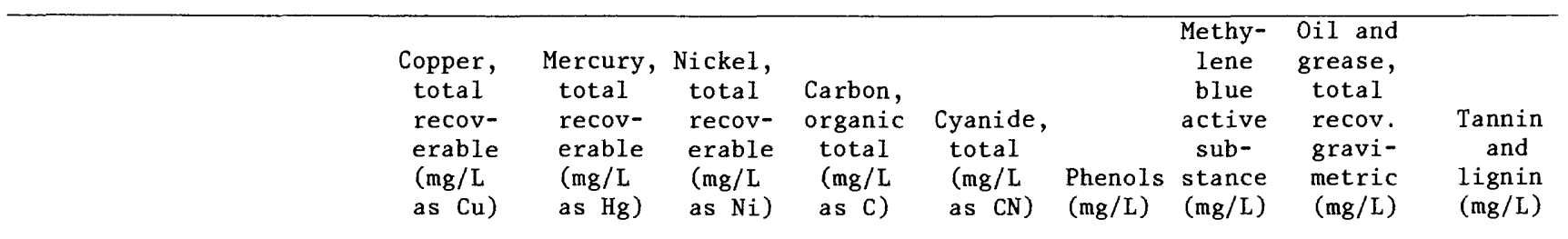

NEW RIVER AT INTERNATIONAL BOUNDARY, AT CALEXICO--Continued

\begin{tabular}{lrrrrrrrrr}
\hline Number of observations & 3 & 3 & 3 & 3 & 3 & 3 & 3 & 3 & 3 \\
Arithmetic mean & 2.8 & 0.03 & 7.3 & 33 & 0.003 & 19 & 1.8 & 8.3 & 2.6 \\
EPA criteria & $(1)$ & 0.05 & $(1)$ & -- & 0.005 & $(2)$ & -- & $(3)$ & -- \\
Number of times exceeded & -- & 1 & -- & -- & 1 & 3 & -- \\
\hline
\end{tabular}

NEW RIVER AT LYONS CROSSING--Continued

\begin{tabular}{|c|c|c|c|c|c|c|c|c|c|}
\hline Number of observations & 3 & 3 & 3 & 3 & 3 & 3 & 3 & 3 & 3 \\
\hline Arithmetic mean & 24 & 0.13 & 5.6 & 21 & 0.003 & 6.6 & 1.96 & 2 & 1.3 \\
\hline EPA criteria & (1) & 0.05 & (1) & - & 0.005 & (2) & - & (3) & -- \\
\hline Number of times exceeded & -- & 1 & -- & -- & 1 & 2 & -- & 3 & -- \\
\hline
\end{tabular}

NEW RIVER AT KEYSTONE--Continued

\begin{tabular}{lrrrrrrrr}
\hline Number of observations & 2 & 2 & 2 & 2 & 2 & 2 & 2 & 2 \\
Arithmetic mean & 20 & 0.05 & 7.3 & 18 & 0.005 & 5 & 0.65 & 0 \\
EPA criteria & $(1)$ & 0.05 & $(1)$ & -- & 0.005 & $(2)$ & -- & $(3)$ \\
Number of times exceeded & - & 1 & -- & -- & 1 & 1 & -- \\
\hline
\end{tabular}

NEW RIVER AT OUTLET NEAR WESTMORLAND--Continued

\begin{tabular}{lrrrrrrrrr}
\hline Number of observations & 3 & 3 & 3 & 3 & 3 & 3 & 3 & 3 & 3 \\
Arithmetic mean & 18 & 0.06 & 12 & 18 & 0.003 & 1.6 & 0.53 & 0 & 0.53 \\
EPA criteria & $(1)$ & 0.05 & $(1)$ & -- & 0.005 & $(2)$ & -- & $(3)$ & -- \\
Number of times exceeded & - & 1 & -- & -- & 1 & 2 & -- & 0 \\
\hline
\end{tabular}

ALAMO RIVER AT OUTLET NEAR NILAND--Continued

\begin{tabular}{|c|c|c|c|c|c|c|c|c|c|}
\hline Number of observations & 3 & 3 & 3 & 2 & 3 & 3 & 3 & 3 & 3 \\
\hline Arithmetic mean & 20 & 0.03 & 12 & 10 & 0.033 & 15 & 0.10 & 0 & 0.53 \\
\hline EPA criteria & (1) & 0.05 & (1) & -- & 0.005 & (2) & -- & (3) & -- \\
\hline Number of times exceeded & -- & 1 & -- & -- & 2 & 2 & -- & 0 & -- \\
\hline
\end{tabular}

See footnotes at end of table.

tion at selected points in a reach, the cumulative effect on the health of the river of the processes described above can clearly be seen.

Longitudinal DO profiles (referred to hereinafter as profiles) were measured on the New River using two different sampling techniques. The first involved measurement of DO concentration at selected bridge crossings as well as above and below the three drop structures. Seven of these measurements were made. The second type was a profile measured from a small boat during its upstream traverse from the Westmorland gage to Highway 80 during the week of September 26-30, 1977 (see fig. 10). For the remaining distance to Calexico, samples were col- lected from bridge crossings. Measurements for this profile were made at 67 sites, with site selections made on the basis of distance and amount of tributary inflow. Sites were located on U.S. Geological Survey quadrangle maps with river morphology, bridge crossings, and contour lines as reference points. Only one profile of this type was made.

DO profiles were measured seasonally by the California Regional Water Quality Control Board, Colorado River Basin Region, to determine the effects of water temperatures on DO concentration (figs. 7 and 8). On December 28, 1976, DO concentrations at the Calexico gage were greater than $9 \mathrm{mg} / \mathrm{L}$, in itself not 
Table 6. Chemical and physical analyses of water, 1978-79, at selected sites-Continued

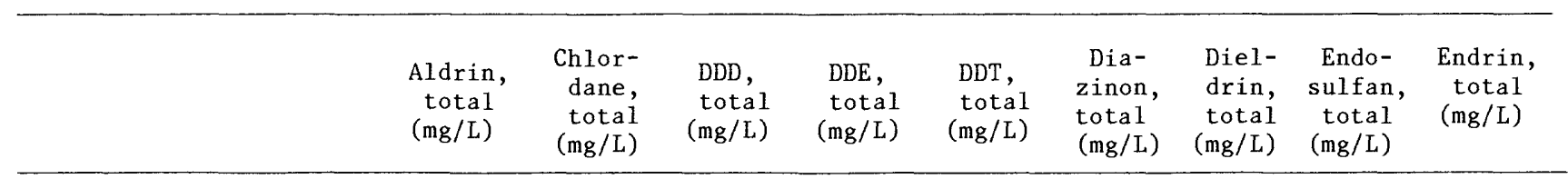

NEW RIVER AT INTERNATIONAL BOUNDARY, AT CALEXICO--Continued

\begin{tabular}{|c|c|c|c|c|c|c|c|c|c|}
\hline Number of observations & 3 & 3 & 3 & 3 & 3 & 3 & 3 & 3 & 3 \\
\hline Arithmetic mean & 0.007 & 0.03 & 0.04 & 0.017 & 0.05 & 0.13 & 0.003 & 0 & 0 \\
\hline EPA criteria & 0.003 & 0.01 & -- & -- & 0.001 & -- & 0.003 & 0.003 & 0.2 \\
\hline Number of times exceeded & 1 & 1 & -- & -- & 3 & -- & 1 & 0 & 0 \\
\hline
\end{tabular}

NEW RIVER AT LYONS CROSSING--Continued

\begin{tabular}{|c|c|c|c|c|c|c|c|c|c|}
\hline Number of observations & 2 & 2 & 2 & 2 & 2 & 2 & 2 & 2 & 2 \\
\hline Arithmetic mean & 0.005 & 0.05 & 0.035 & 0.015 & 0.02 & 0.13 & 0.01 & 0 & 0 \\
\hline EPA criteria & 0.003 & 0.01 & -- & -- & 0.001 & -- & 0.003 & 0.003 & 0.2 \\
\hline Number of times exceeded & 1 & 1 & -- & -- & 2 & -- & 2 & 0 & 0 \\
\hline
\end{tabular}

NEW RIVER AT KEYSTONE--Continued

\begin{tabular}{|c|c|c|c|c|c|c|c|c|c|}
\hline Number of observations & 3 & 3 & 3 & 3 & 3 & 3 & 3 & 3 & 3 \\
\hline Arithmetic mean & 0 & 0 & 0.013 & 0.006 & 0.013 & 0.073 & 0.01 & 0 & 0 \\
\hline EPA criteria & 0.003 & 0.01 & -- & -- & 0.001 & -- & 0.003 & 0.003 & 0.2 \\
\hline Number of times exceeded & 0 & 0 & -- & -- & 1 & -- & 3 & 0 & 0 \\
\hline
\end{tabular}

NEW RIVER AT OUTLET NEAR WESTMORLAND--Continued

\begin{tabular}{|c|c|c|c|c|c|c|c|c|c|}
\hline Number of observations & 5 & 5 & 5 & 5 & 5 & 5 & 5 & 5 & 5 \\
\hline Arithmetic mean & 0.002 & 0 & 0.012 & 0.008 & 0.006 & 0.028 & 0.008 & 0.004 & 0 \\
\hline EPA criteria & 0.003 & 0.01 & -- & -- & 0.001 & - & 0.003 & 0.003 & 0.2 \\
\hline Number of times exceeded & 1 & 0 & -- & -- & 3 & -- & 4 & 2 & 0 \\
\hline
\end{tabular}

ALAMO RIVER AT OUTLET NEAR NILAND--Continued

\begin{tabular}{|c|c|c|c|c|c|c|c|c|c|}
\hline Number of observations & 5 & 5 & 5 & 5 & 5 & 5 & 5 & 5 & 5 \\
\hline Arithmetic mean & 0 & 0 & 0.002 & 0.024 & 0.004 & 0.13 & 0.006 & 0.012 & 0.006 \\
\hline EPA criteria & 0.003 & 0.01 & -- & -- & 0.001 & -- & 0.003 & 0.003 & 0.2 \\
\hline Number of times exceeded & 1 & 0 & -- & - & $?$ & - & $?$ & 5 & 0 \\
\hline
\end{tabular}

indicative of an unhealthy environment, but DO concentrations gradually decreased downstream (fig. 7) to: 6.5 $\mathrm{mg} / \mathrm{L}$ at Clark Road, $4.5 \mathrm{mg} / \mathrm{L}$ at Brockman Road, and $2.7 \mathrm{mg} / \mathrm{L}$ at Highway 80 . Deviations from a normal curve are due to variability in organic material, photosynthesis, and tributary inflow. Downstream from Highway 80 , the DO concentration improved rapidly. The rock weir near Seeley added to the reaeration. At Worthington Road (site 26.4), the DO concentration had increased to about 6.5 $\mathrm{mg} / \mathrm{L}$, and to about $8.0 \mathrm{mg} / \mathrm{L}$ at Forrester Road (site 32.9). From Forrester Road to the Salton Sea, increases in DO concentration were due mainly to reaeration by the three drop structures. The highest observed DO concentration of the study, more than $10 \mathrm{mg} / \mathrm{L}$, occurred below Drop 2, the farthest downstream drop structure.
The effect of seasonal variation on the shape of the sag curve becomes apparent in the April 5, 1977, profile. The profile of January 11,1977 , is similar to that of December 28, although the initial decrease is more pronounced. The shape of the April 5, 1977, profile, however, is different from either of the winter profiles (fig. 7). Although the initial concentration at the Calexico gage was $4.5 \mathrm{mg} / \mathrm{L}$, at $\mathrm{Kemp}$ Road, $1.5 \mathrm{mi}$ downstream, the DO concentration was zero. Anaerobic conditions persisted for $4 \mathrm{mi}$ to Clark Road. From Highway 80 to Forrester Road, DO concentration increased. Reaeration by the rock weir near Seeley accounted for the instantaneous increase in DO concentration. In the lower reaches of the river, reaeration from the three drop structures added to the DO concentration so that by Lack Road (site 60.2) 
Table 6. Chemical and physical analyses of water, 1978-79, at selected sites-Continued

\begin{tabular}{|c|c|c|c|c|c|c|c|c|}
\hline $\begin{array}{l}\text { Hepta- } \\
\text { chlor, } \\
\text { total } \\
(\mathrm{mg} / \mathrm{L})\end{array}$ & $\begin{array}{l}\text { Hepta- } \\
\text { chlor, } \\
\text { epox- } \\
\text { ide, } \\
\text { total } \\
\text { (mg/L) }\end{array}$ & $\begin{array}{l}\text { Lin- } \\
\text { dane, } \\
\text { total } \\
(\mathrm{mg} / \mathrm{L})\end{array}$ & $\begin{array}{c}\text { Mala- } \\
\text { thion, } \\
\text { total } \\
(\mathrm{mg} / \mathrm{L})\end{array}$ & $\begin{array}{r}\text { Methyl } \\
\text { para- } \\
\text { thion, } \\
\text { total } \\
(\mathrm{mg} / \mathrm{L})\end{array}$ & $\begin{array}{c}\text { Methyl } \\
\text { tri- } \\
\text { thion, } \\
\text { total } \\
(\mathrm{mg} / \mathrm{L})\end{array}$ & $\begin{array}{r}2,4-D \\
\text { total } \\
(\mathrm{mg} / \mathrm{L})\end{array}$ & $\begin{array}{c}2,4,5-\mathrm{T} \\
\text { total } \\
(\mathrm{mg} / \mathrm{L})\end{array}$ & $\begin{array}{l}\text { Mirex, } \\
\text { total } \\
(\mathrm{mg} / \mathrm{L})\end{array}$ \\
\hline
\end{tabular}

NEW RIVER AT INTERNATIONAL BOUNDARY, AT CALEXICO--Continued

\begin{tabular}{|c|c|c|c|c|c|c|c|c|c|}
\hline Number of observations & 3 & 3 & 3 & 3 & 3 & 3 & 2 & 2 & 3 \\
\hline Arithmetic mean & 0 & 0 & 0.02 & 0.06 & 0 & 0 & 0 & 0 & 0 \\
\hline EPA criteria & 0.001 & -- & 0.01 & 0.1 & -- & -- & -- & $\cdots$ & 0.001 \\
\hline Number of times exceeded & 0 & $\ldots$ & 1 & 1 & $\ldots$ & $\ldots$ & - & -- & 0 \\
\hline
\end{tabular}

NEW RIVER AT LYONS CROSSING--Continued

\begin{tabular}{|c|c|c|c|c|c|c|c|c|c|}
\hline Number of observations & 2 & 2 & 1 & 2 & 2 & 2 & 2 & 2 & 2 \\
\hline Arithmetic mean & 0 & 0 & 0 & 0.04 & 0 & 0 & 0 & 0 & 0 \\
\hline EPA criteria & 0.001 & -- & 0.01 & 0.1 & -- & -- & -- & - & 0.001 \\
\hline Number of times exceeded & 0 & -- & 0 & 0 & -- & - & -- & -- & 0 \\
\hline
\end{tabular}

NEW RIVER AT KEYSTONE--Continued

\begin{tabular}{|c|c|c|c|c|c|c|c|c|c|}
\hline Number of observations & 3 & 3 & 3 & 3 & 3 & 3 & 3 & 3 & 3 \\
\hline Arithmetic mean & 0 & 0 & 0 & 0.17 & 0.9 & 0 & 0.38 & 0 & 0 \\
\hline EPA criteria & 0.001 & - & 0.01 & 0.1 & -- & - & $\cdots$ & -- & 0.001 \\
\hline Number of times exceeded & 0 & -- & 0 & 1 & -- & - & -- & -- & 0 \\
\hline
\end{tabular}

NEW RIVER AT OUTLET NEAR WESTMORLAND--Continued

\begin{tabular}{|c|c|c|c|c|c|c|c|c|c|}
\hline Number of observations & 5 & 5 & 5 & 5 & 5 & 5 & 5 & 5 & 5 \\
\hline Arithmetic mean & 0 & 0 & 0.004 & 0.044 & 0 & 0 & 0.35 & 0 & 0 \\
\hline EPA criteria & 0.001 & $\infty$ & 0.01 & 0.1 & $\cdots$ & - & -- & -- & 0.001 \\
\hline Number of times exceeded & 0 & -. & 0 & 2 & -- & -. & -- & -- & 0 \\
\hline
\end{tabular}

ALAMO RIVER AT OUTLET NEAR NILAND--Continued

\begin{tabular}{|c|c|c|c|c|c|c|c|c|c|}
\hline Number of observations & 5 & 5 & 5 & 5 & 5 & 5 & 5 & 5 & 5 \\
\hline Arithmetic mean & 0 & 0 & 0.008 & 0.006 & 0.20 & 0 & 0.52 & 0 & 0 \\
\hline EPA criteria & 0.001 & $\cdots$ & 0.01 & 0.1 & $\cdots$ & $\cdots$ & -- & - & 0.001 \\
\hline Number of times exceeded & 0 & - & 1 & 0 & $\cdots$ & - & -- & -- & 0 \\
\hline
\end{tabular}

the DO concentration was about $8 \mathrm{mg} / \mathrm{L}$. In general, the April data reflect the effects of temperature on the sag curve, resulting in sharper decreases in DO concentration immediately downstream from the international boundary, a longer zone of depressed conditions, greater reaeration at the drop structures because of the larger deficit, and an overall lowering of DO concentration in the river.

To determine the effects of high water temperatures and high organic levels on DO concentrations, three profiles were measured by the California Regional Water Quality Control Board, Colorado River Basin Region, during the summer of 1977. The effects were extreme, especially on July 28 (fig. 8). Water entering the United States in the New River was devoid of dissolved oxygen.
The zone of depletion extended from the Calexico gage to Worthington Road, $26.4 \mathrm{mi}$ downstream. Although some recovery occurred by Forrester Road, that is, the DO concentration increased to $2 \mathrm{mg} / \mathrm{L}$, conditions were still indicative of poor water quality. Reaeration from the rock weir near Seeley, which helped to add dissolved oxygen increases during the winter profiles to bring a return to aerobic conditions, did not add sufficient dissolved oxygen during the summer profiles to overcome the anaerobic conditions of the river.

In the lower reaches of the river, reaeration caused in part by drop structures increased the DO concentration. Immediately downstream from these structures, the DO concentration decreased, indicating that reaeration from 
Table 6. Chemical and physical analyses of water, 1978-79, at selected sites-Continued

\begin{tabular}{ccccc} 
Per- & Silvex, & Toxa- & Naphthalenes & PCB, \\
thane, & total \\
total & tone, & polychlo- \\
$(\mathrm{mg} / \mathrm{L})$ & $(\mathrm{mg} / \mathrm{L})$ & $\begin{array}{c}\text { totated, } \\
(\mathrm{mg} / \mathrm{L})\end{array}$ & $\begin{array}{c}\text { total } \\
\text { (mg/L) }\end{array}$ & $(\mathrm{mg} / \mathrm{L})$ \\
\hline
\end{tabular}

NEW RIVER AT INTERNATIONAL BOUNDARY, AT CALEXICO--Continued

\begin{tabular}{lrrrrr}
\hline Number of observations & 3 & 2 & 3 & 1 & 3 \\
Arithmetic mean & 0 & 0 & 0 & 0 & 0.15 \\
EPA criteria & -- & -- & 0.005 & -- & 0.001 \\
Number of times exceeded & -- & -- & 0 & -- & 2 \\
\hline
\end{tabular}

NEW RIVER AT LYONS CROSSING--Continued

\begin{tabular}{|c|c|c|c|c|c|}
\hline Number of observations & 2 & 2 & 2 & 0 & 2 \\
\hline Arithmetic mean & 0 & 0 & 0 & -- & 0 \\
\hline EPA criteria & -- & -- & 0.005 & -- & 0.001 \\
\hline Number of times exceeded & -- & -- & 0 & -- & 0 \\
\hline
\end{tabular}

NEW RIVER AT KEYSTONE--Continued

\begin{tabular}{|c|c|c|c|c|c|}
\hline Number of observations & 3 & 3 & 3 & 0 & 3 \\
\hline Arithmetic mean & 0 & 0.063 & 0 & -- & 0 \\
\hline EPA criteria & -- & -- & 0.005 & -- & 0.001 \\
\hline Number of times exceeded & -- & -- & 0 & -- & 0 \\
\hline
\end{tabular}

NEW RIVER AT OUTLET NEAR WESTMORLAND--Continued

\begin{tabular}{|c|c|c|c|c|c|}
\hline Number of observations & 5 & 5 & 5 & 2 & 5 \\
\hline Arithmetic mean & 0 & 0.052 & 0 & 0 & 0.02 \\
\hline EPA criteria & -- & - & 0.005 & -- & 0.001 \\
\hline Number of times exceeded & -- & -- & 0 & -- & 1 \\
\hline
\end{tabular}

ALAMO RIVER AT OUTLET NEAR NILAND--Continued

\begin{tabular}{lrrrrr}
\hline Number of observations & 5 & 5 & 5 & 2 & 5 \\
Arithmetic mean & 0 & 0.22 & 0 & 0 & 0 \\
EPA criteria & -- & -- & 0.005 & -- & 0.001 \\
Number of times exceeded & -- & -- & 0 & -- & 0 \\
\hline
\end{tabular}

1Fish species dependent.

2Fish flesh tainting.

3Fish species dependent, but should be virtually free from floating accumulations.

these structures was insufficient to satisfy the oxygen demand of the river. From Drop 2 to Lack Road, a distance of about $11 \mathrm{mi}$, DO concentration decreased from 7.5 to $4.5 \mathrm{mg} / \mathrm{L}$, the lowest observed concentration for that reach. Not only was the zone of DO depletion in the upper 26-mile reach greater than for any of the other profiles (compare figs. 7 and 8), but the lower reaches of the river were degraded to the extent that reaeration was insufficient to maintain concentrations of dissolved oxygen necessary to support a varied fish population.

The most detailed of the profiles was that of September $26-30,1977$, in which measurements were made at 67 sites, reached by boat (fig. 9). This profile showed the reaeration effect of the rock weir near Seeley as well as the effect of photosynthesis on the shape of the profile (fig. 10). Because measurements at selected bridge crossings were made in the middle of the day and usually took about 5 hours to complete, the effects of photosynthesis were not as pronounced as for this sampling, which began early in the morning and lasted until evening each day.

Photosynthetic effects are seen in the sharp increases in DO concentration at Salt Creek (site 25.6) and Keystone Road (site 36.1) (fig. 10 and table 7). The measurements immediately downstream of these sites were made 
in the afternoon when photosynthetic activity and DO concentration were high. The lower concentrations at or just upstream from these sites were measured in the early morning, when photosynthetic activities were low and net dissolved oxygen produced during the day had been metabolized by respiration throughout the night. The difference between the late afternoon and early morning DO concentration at the same site is an indication of abundant periphyton and submerged plant communities. At Salt Creek the difference was $2.8 \mathrm{mg} / \mathrm{L}$ and at Keystone Road about $2 \mathrm{mg} / \mathrm{L}$, indicating the presence of a biotic community that is actively photosynthesizing during the daylight hours. For comparison, the Los Angeles River at Willow Street, Los Angeles County (concrete-lined channel with low-flow notch), with an extensive periphyton community has afternoon DO concentrations as high as $22 \mathrm{mg} / \mathrm{L}$ at $300-$ percent saturation and early morning concentrations less than $1 \mathrm{mg} / \mathrm{L}$. In the downstream reaches of the New River, more than 50 percent of the flow is agricultural return, which has a high silt concentration. The high silt concentrations inhibit light penetration and photosynthesis, thus limiting the periphyton and plant communities.

\section{Diel Variations}

In order to assess further the effects of organic material on the intensity of biological activity, two separate diel (24-hour) studies were made. The first was completed during the week of October 17, 1977, and the second during the week of April 17, 1978. For the October study, hourly measurements of DO concentration, $\mathrm{pH}$, water temperature, and specific conductance were made at the following sites on the New River:

\section{At international boundary, at Calexico (site 0) \\ At Highway 80 (site 19.5) \\ At Keystone Road (site 36.1) \\ Below Drop 4 near Brawley (site 43.6) \\ At outlet near Westmorland (site 61.2).}

At the Calexico gage and at Highway 80 , significant variations in DO concentration were observed (figs. 11 and 12). On October 17, at 1300 hours, the DO concentration at the Calexico gage was $3 \mathrm{mg} / \mathrm{L}$ and 36 -percent saturation. Seven hours later, the river became anaerobic. These conditions remained in effect for 9 hours, then gradually improved. By the end of the diel study the DO concentration had increased from 0 to $2 \mathrm{mg} / \mathrm{L}$, probably owing to photosynthesis with the resumption of sunlight. Apparently a pollutant slug containing high concentrations of organic matter had passed by the Calexico gage. The slug, along with respiration of aquatic plants and animals and oxidation of benthal organic deposits, must have exerted an immediate oxygen demand on the water in order to produce such a rapid decline in DO concentration. If the slug contained sewage which had turned anaerobic when discharged into the river, the reduced compounds in that slug would place an immediate oxygen demand on the New River, thus accounting for the steepness of the decline. Another possibility would be an industrial effluent that contained chemical compounds in a reduced state. The effect would be the same as for the sewage although the recovery pattern would be different.

DO concentration at the Highway 80 crossing also reflected the effects of the slug discharge. At 1400 hours on October 18, after 18-22 hours of travel from Calexico, the pollutant slug that caused the abrupt decline in DO concentration at Calexico reached the Highway 80 site. Upon its arrival, the DO concentration decreased to 0.1 $\mathrm{mg} / \mathrm{L}$ (fig. 12). The 18-22 hours agree with the measured traveltime determined from time-of-travel study. Thus, the effects of the pollutant slugs originating south of the international boundary can still be large at least $20 \mathrm{mi}$ downstream. Because the diel measurements at Highway 80 and at Keystone Road were made during the same time frame and the low DO concentrations were observed at the end of the 24-hour period at Highway 80 , it is not certain whether the effects of the pollutant slug would have been detected at the Keystone Bridge site, $16.6 \mathrm{mi}$ and 15 hours downstream from Highway 80 .

Because agricultural effluent exerts an oxygen demand, DO concentrations observed at sites downstream from Calexico may not be due entirely to the organic loads at the international boundary and the subsequent aerobic or anaerobic oxidation of those loads. For the reach between Calexico and Highway 80, the gain in discharge from agricultural drains is small. Downstream from Highway 80 , however, the discharge from agricultural drains increases, apparently diluting organic loads as evidenced by the DO profiles downstream from Highway 80 .

The pattern of DO concentration throughout the diel study at the Keystone site was similar to that in other eutrophic streams. The highest concentrations were observed in the late afternoon as a result of the photosynthetic activity of the algae, but the lowest concentrations were observed in the early morning as a result of respiration. The highest observed concentration of $6.5 \mathrm{mg} / \mathrm{L}$ and 80-percent saturation was at 1600 hours. Concentrations were generally lowest between 0100 to 0200 hours, although the lowest observed concentration of $4.7 \mathrm{mg} / \mathrm{L}$ was at 0900 hours. Deviations from the normal diel pattern resulted from ever-changing pollutant loads, variable flow at the international boundary, and irrigation return flow. Downstream, below Drop 4 near Brawley, DO concentrations showed very little variation. Percent saturation was near 85 , with DO concentrations of about $7 \mathrm{mg} / \mathrm{L}$. At the Westmorland site, diel variations were fairly normal. The highest concentration of $6.4 \mathrm{mg} / \mathrm{L}$ and 78 -percent saturation was at 1600 hours, and the lowest concentration 


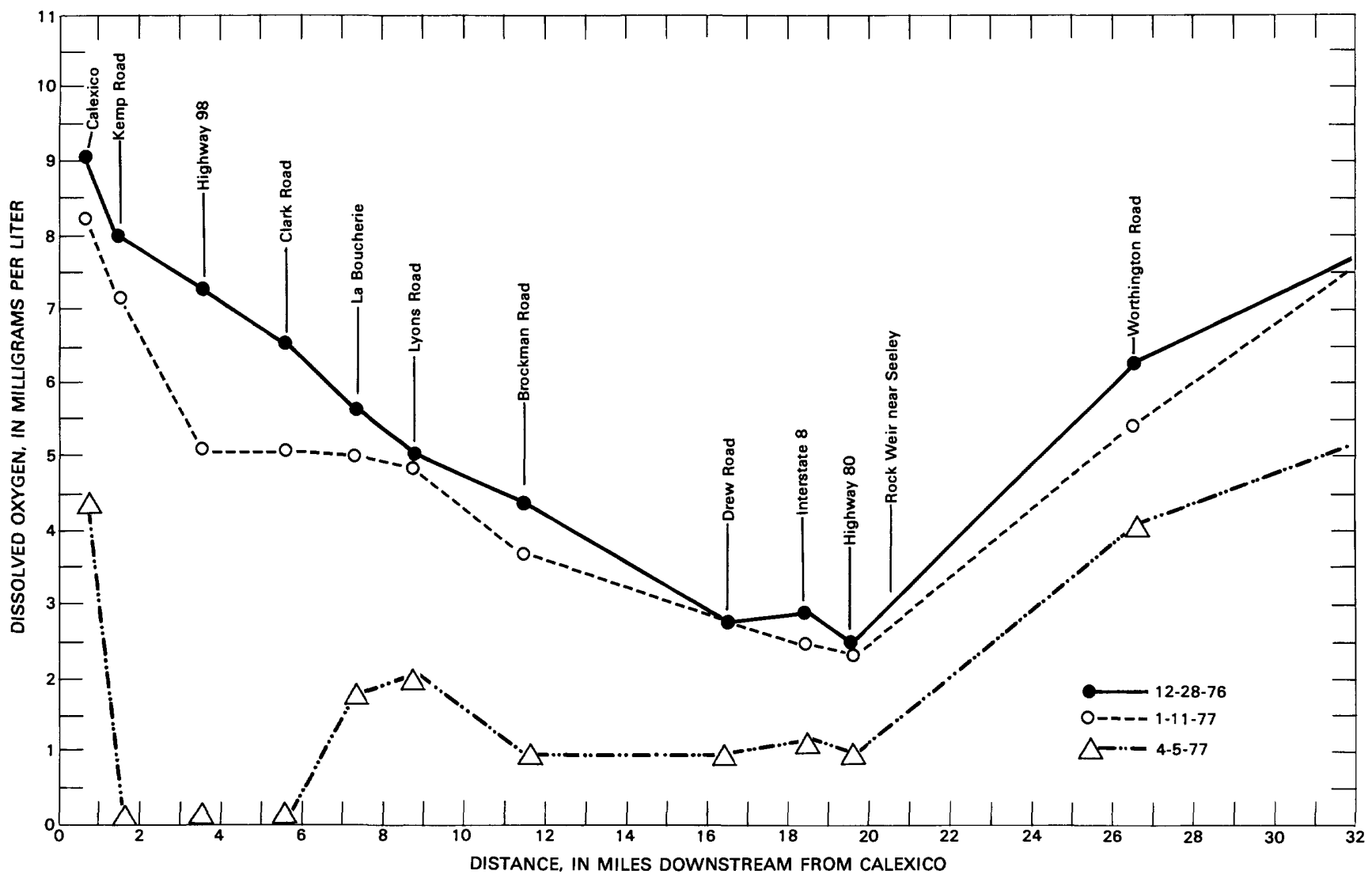

Figure 7. Dissolved-oxygen concentration at selected sites on the New River, December 28, 1976, January 1, and April 5, 1977. Data from California Regional Water Quality Control Board, Colorado River Basin Region (written commun., 1978).

at $4.9 \mathrm{mg} / \mathrm{L}$ and $60-$ percent saturation was at 0500 hours.

During the week of April 17, 1978, measurements were made hourly throughout $2 \frac{1}{2}$ days at five sites on the New River. The start of sampling was determined from the time-of-travel data. As the leading edge of the water sampled at the first site arrived at the second site, sampling began. This same pattern was used for the remainder of the New River sites. For comparison, measurements were made throughout one diel period at the Alamo River at the outlet near Niland, the control site. Measurements were made at the following New River sites:

International boundary at Calexico (site 0 )

Clark Road (site 5.5)

Lyons Crossing (site 8.8)

Highway 80 (site 19.5)

At outlet near Westmorland gage (site 61.2).

The DO concentration curve at the Calexico gage began to increase about sunrise, reaching its maximum concentration in the afternoon (fig. 13). On April 17, the maximum concentration was $5.2 \mathrm{mg} / \mathrm{L}$ and 60 -percent saturation at 1300 hours. On the following day, it was 5.8 $\mathrm{mg} / \mathrm{L}$ and $70-$ percent saturation at 1500 hours. As the sunlight decreased, DO concentration also decreased, reaching its lowest concentration during the late nightearly morning hours. Concentrations of DO reached a minimum of $0.5 \mathrm{mg} / \mathrm{L}$ between 2200 hours and 2400 hours on April 17, and on April 18 the minimum concentration of $0.1 \mathrm{mg} / \mathrm{L}$ occurred between 2200 hours on the 18 th and 0100 hours the next day. The difference between the maximum and minimum DO concentration of almost $6 \mathrm{mg} / \mathrm{L}$ on April 18 indicates that an abundance of plantlife existed at the Calexico gage. Water at this site is usually free of the high silt concentrations that are characteristic at downstream sites and are a result of agricultural return. With background turbidities of about 28 JTU's (Jackson turbidity unit) and with a depth of about $3 \mathrm{ft}$, there is sufficient light for photosynthesis to occur throughout the water column and on the riverbed at the Calexico gage.

The next two sites, Clark Road and Lyons Crossing, had similar patterns but not as pronounced day-night amplitude of DO concentration. Diel dissolved-oxygen concentration and percent saturation curves are shown for five sites on the New River and the control site on the Alamo River (fig. 14). Concentrations were depressed at Clark Road from 1900 hours April 17 until 1000 hours 


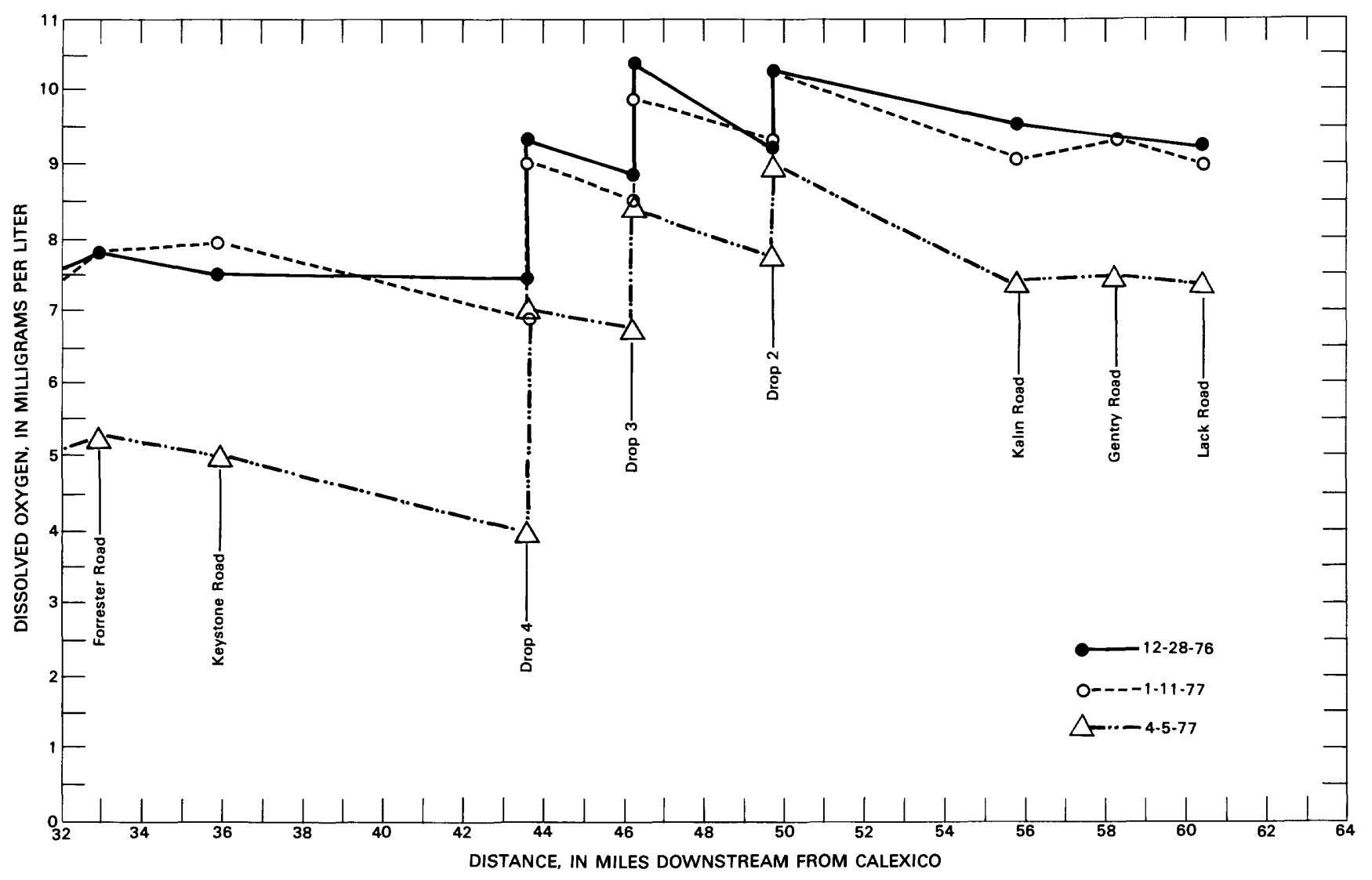

Figure 7. Dissolved-oxygen concentration at selected sites on the New River, December 28, 1976, January 1, and April 5, 1977.-Continued.

the next day. At Lyons Crossing the decrease began in the late afternoon of the 17 th, as would be expected in a diel study such as described for Calexico. The DO concentration, however, remained depressed until 1130 hours April 18, at which time the DO meter malfunctioned. Both sites showed a rise in DO concentration that began at sunrise April 19 and reached maximum concentrations in the afternoon.

Downstream sites did not develop any major diel variations in DO concentration throughout the $2^{1 / 2}$ days of measurements (fig. 14). At Highway 80 the DO concentration was about $3 \mathrm{mg} / \mathrm{L}$ throughout the study and did not present any discernible pattern. The Westmorland site also showed little variation in DO concentration, averaging about $6.4 \mathrm{mg} / \mathrm{L}$ with percent saturation in the high 60 's. The difference between the maximum and minimum observed DO concentrations was $2 \mathrm{mg} / \mathrm{L}$. The control site on the Alamo River at outlet also showed little variation throughout the diel study. The average DO concentration was about $7.4 \mathrm{mg} / \mathrm{L}$, with percent saturation in the middle 80 's. The difference between the maximum and minimum concentrations was $1.3 \mathrm{mg} / \mathrm{L}$. The absence of fluctuation in DO concentration at Highway 80 and the Westmorland gage indicated that plant growth was minimal during the sampling period. Because plant growth was minimal, photosynthesis and respiration did not exert a heavy influence on the DO equilibrium of the river. The Alamo River, which also had little variation in DO concentration, did not support an extensive periphyton or phytoplankton population.

\section{EPA Criteria}

According to the criteria established by EPA (1976, p. 123), the minimum DO concentration necessary to maintain good fish populations is $5.0 \mathrm{mg} / \mathrm{L}$. "Water should contain sufficient dissolved oxygen to maintain aerobic conditions in the water column," the EPA states. Based on both the profile and diel data, it is evident that in the upper reaches of the New River, the $5.0 \mathrm{mg} / \mathrm{L}$ criterion is seldom met. The lower reaches afford a more suitable environment for fish populations, but even there, during the summer months, the fish population would tend to be stressed. The observations of fish kills, as noted in the introduction, are well documented. Often it is the extremes in conditions, not the mean, that lead to biological stress. For fish in the upper reaches of the New River this would be especially true. 


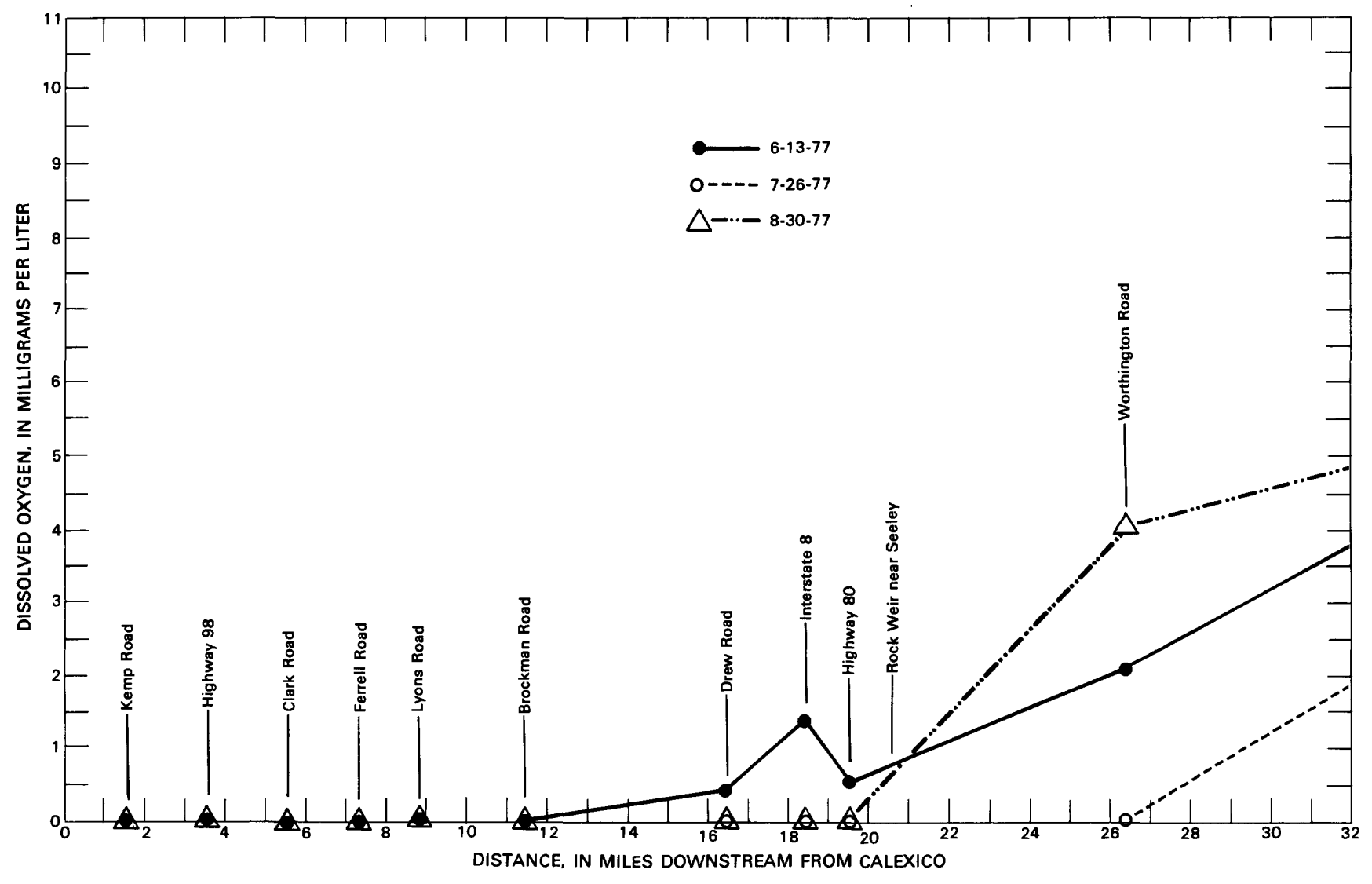

Figure 8. Dissolved-oxygen concentration at selected sites on the New River, June 13, July 28, and August $30,1977$. Data from California Regional Water Quality Control Board, Colorado River Basin Region (written commun., 1978).

\section{Organic Material}

Excessive organic material rapidly decomposed by bacteria and other organisms was documented as the major problem in the New River. This decomposition by oxidation uses the available oxygen in the river and produces the effects discussed in the previous section.

The frequency of occurrence of excessive organic material is shown by distribution graphs of chemical and biochemical oxygen demand (fig. 15). These graphs represent a composite of data collected by the U.S. Geological Survey and the California Regional Water Quality Board, Colorado River Basin Region, at the Calexico gage during the period July 1976 to March 1978. The right skewness of the data indicates that these constituents are best represented by geometric means. For the data presented in the graph, the geometric mean concentration for COD was $133 \mathrm{mg} / \mathrm{L}$, compared with $322 \mathrm{mg} / \mathrm{L}$ for the samples collected during the reconnaissance. The geometric mean concentration for biochemical oxygen demand (BOD) was $44 \mathrm{mg} / \mathrm{L}$. The correlation coefficient between BOD and COD was 0.9 (a coefficient of 1.0 indicates a perfect relation between variables). There was almost no correlation between DO and BOD, as indicated by a correlation coefficient of -0.20 . This lack of correlation is due in part to both temperature and time. Temperature affects the saturation concentration of oxygen and the BOD reaction rates, and time controls the degree to which the BOD is satisfied by oxidation. Because the Calexico sampling site is immediately downstream from the international boundary, there is insufficient time for variations in BOD to affect DO concentration. In addition, the river is often stressed to the extent that it is devoid of dissolved oxygen, and additional organic loads would have little or no effect on the near zero DO concentration.

\section{Oxidation of Organics}

The presence of organic matter in water is detected in a variety of ways, each indicative of different characteristics of the organic substance. The main indicators used in this study were BOD, COD, and total, dissolved, and suspended organic carbon. BOD reflects the amount of oxygen consumed by biochemical oxidation of organic matter in 5 days. COD reflects a high temperature, wet oxidation of the organic matter by a strong oxidizing agent. Oxidizable compounds other than organic matter 


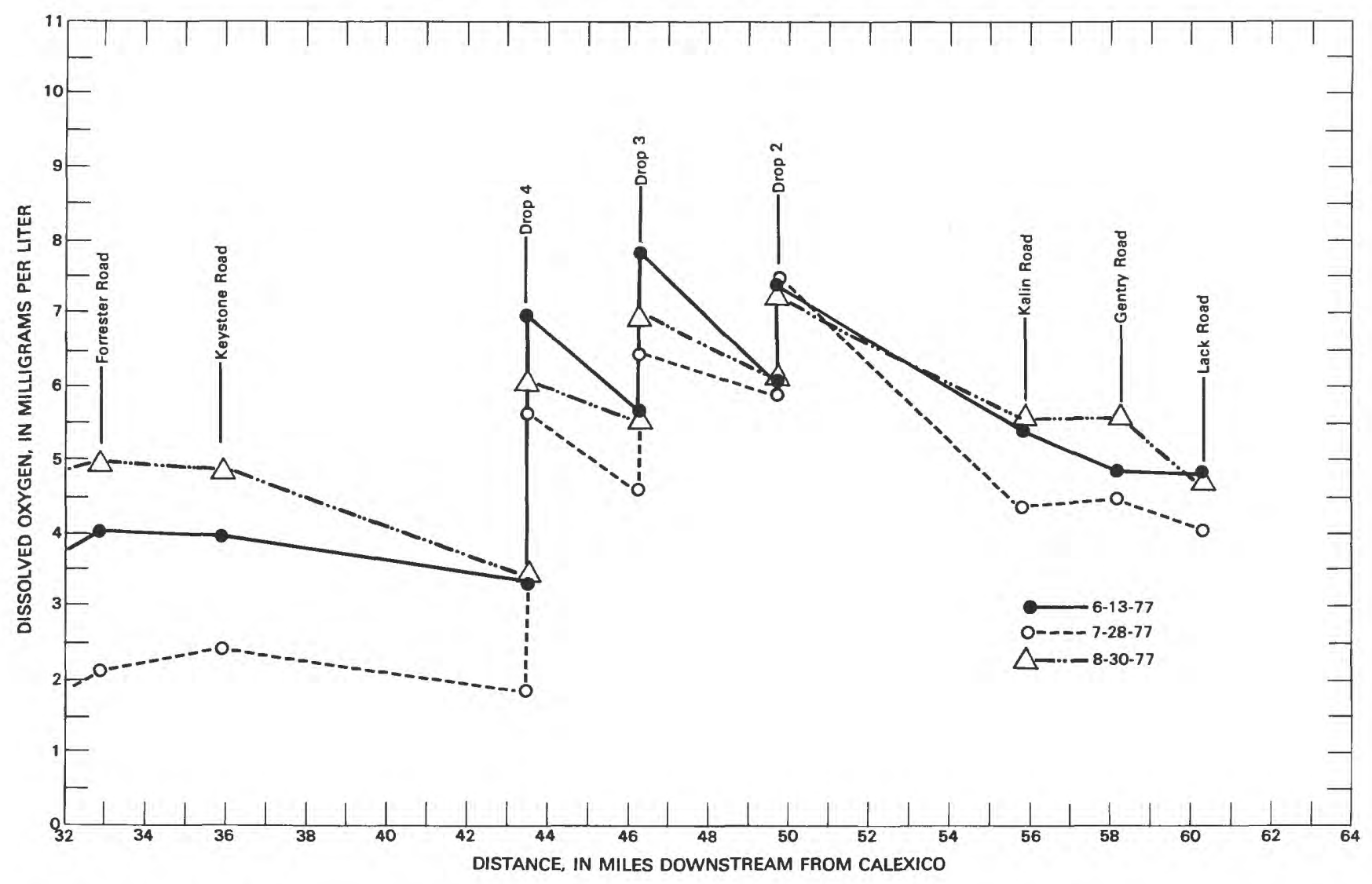

Figure 8. Dissolved-oxygen concentration at selected sites on the New River, June 13, July 28, and August 30, 1977.Continued.

are also included in this determination. The relation between $\mathrm{BOD}$ and $\mathrm{COD}$ was shown in the reconnaissance. The magnitude of the difference between BOD and COD, having geometric means of $44 \mathrm{mg} / \mathrm{L}$ versus $133 \mathrm{mg} / \mathrm{L}$, shows how little of the chemically oxidizable material can be biochemically oxidized in 5 days. Another set of indicators of organic pollution are total, dissolved, and suspended organic carbon. These determinations measure all the organic carbon in the respective phases regardless of its oxidation state, and are gross indicators of organic content but are not useful for predicting oxygen demand unless the nature of the pollutant is very consistently the same.

The two main processes that deal with organic matter in the aqueous environment are aerobic and anaerobic oxidation. In aerobic oxidation, oxygen dissolved in the water acts as the oxidizing agent (electron acceptor) and in turn is reduced, usually to carbon dioxide that can form bicarbonate or carbonate ions. The organic matter goes from a reduced to an oxidized state. In anaerobic oxidation, combined oxygen in the form of organic or inorganic compounds is used as the electron acceptor, and in turn is reduced. During anaerobic oxidation, the nitrates are preferentially reduced to ammonia; then sulfates are re- duced to hydrogen sulfide; and carbon dioxide is reduced to methane. From the observations noted in the introduction, it is apparent that anaerobic oxidation does occur in the upper reaches of the New River, especially from Clark Road to Brockman Road, and hydrogen sulfide is probably produced by the anaerobic oxidation of sulfate.

\section{Reaction Rates}

The effect of temperature on the saturation of water with respect to oxygen was discussed earlier. The effect of temperature on the rate of satisfaction of BOD also contributes to the low summertime DO concentrations that were observed in the profiles. The metabolic rate at which bacteria oxidize organic matter is temperature dependent. For 5-day biochemical oxygen demand $\left(\mathrm{BOD}_{5}\right)$, the reaction rates change in the following manner: At $29^{\circ} \mathrm{C}, \mathrm{BOD}$ half-life is 2 days with a reaction rate of 82 percent per day; at $20^{\circ} \mathrm{C}, \mathrm{BOD}$ half-life is 3 days with a reaction rate of 68 percent per day; and at $14^{\circ} \mathrm{C}$, BOD half-life is 4 days with a reaction rate of 58 percent per day (Klein, 1962). Another factor affecting the rate of oxidation is the amount of unoxidized organic material in the water. 


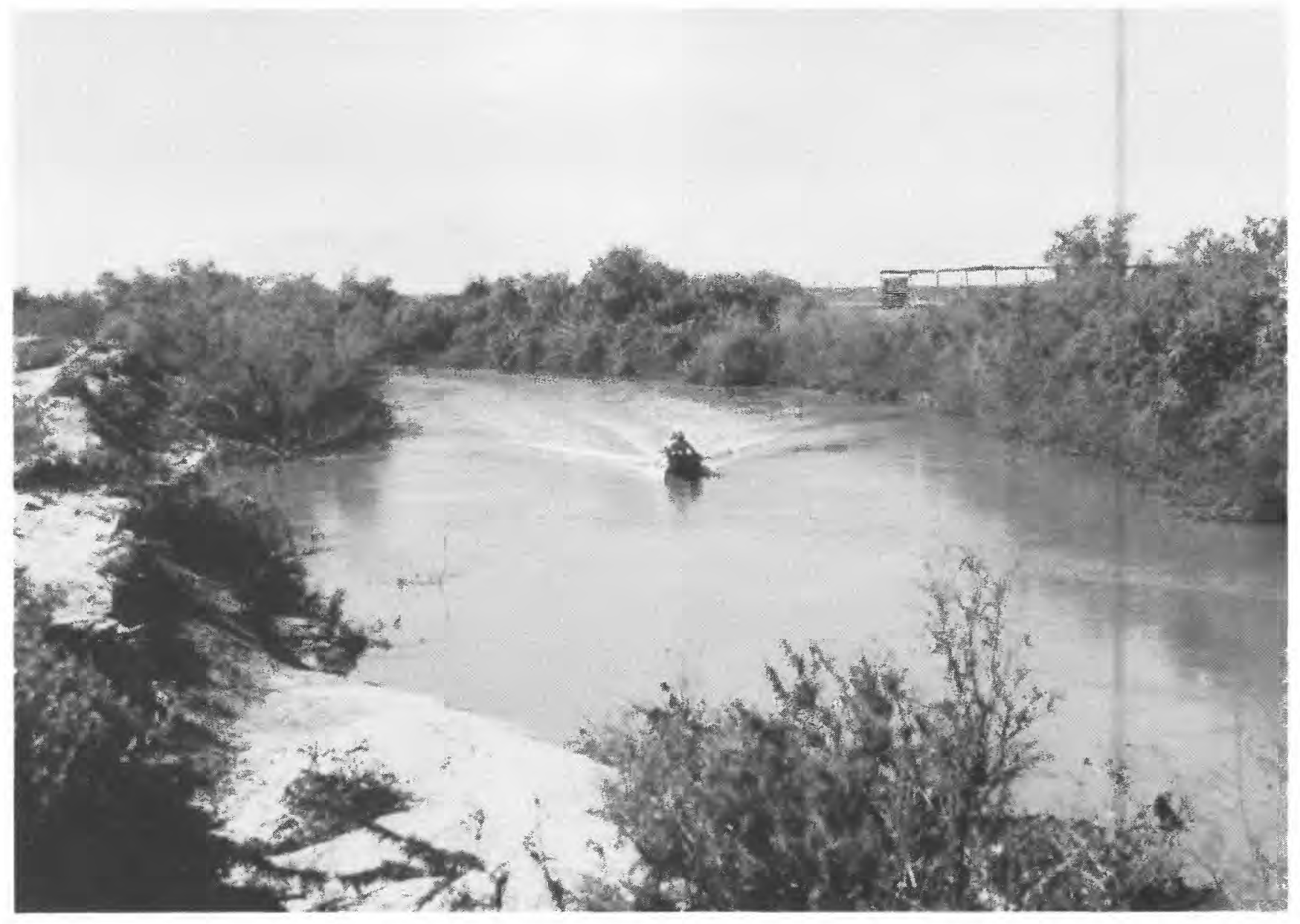

Figure 9. New River upstream of Westmorland gage during longitudinal profiling by boat, September $26-30,1977$.

Phelps' law states that "the rate of biochemical oxidation of organic matter is proportional to the remaining concentration of unoxidized substance measured in terms of its oxidizability" (Velz, 1970, p. 142). This law applies only when there is excess oxygen and a constant temperature. Because the reaction rate is so much faster at high temperatures-when dissolved oxygen is correspondingly lower-high organic loading during the summer months leads to deoxygenation and anaerobic conditions in the river as shown in the profiles of June, July, and August of 1977.

\section{Time-of-Travel Investigations}

By combining the traveltime information with organic concentrations that were determined during the diel study and profile phases, the oxidation processes involved in river purification can be better understood. To determine the rate of migration of the slug discharges, two time-of-travel investigations were made, February 6-9 and
June 19-22, 1978, in the reach between the international boundary and the Salton Sea. Seventy-five pounds of a 20 -percent solution of rhodamine WT fluorescent dye were injected in the river at the Calexico gaging site. Emphasis was placed on determining the leading dye edge and the peak dye concentration at the sites shown in table 8 and figure 16. The average speed of the flow from Calexico to the Westmorland gage was about $1.1 \mathrm{mi} / \mathrm{h}$.

\section{Longitudinal Stream Profiles}

The longitudinal stream profiles made by boat of September 26-30, 1977, and at bridge crossings May 10, 1978 , were the only profiles that involved collection of samples for determination of organic concentrations. During the profile of September 26-30, samples for the determination of BOD, COD, TOC, and DOC, were collected at 20 sites on the New River (table 9). In general, the concentrations of these constituents were higher at the Calexico gage than downstream at the Westmorland gage. 

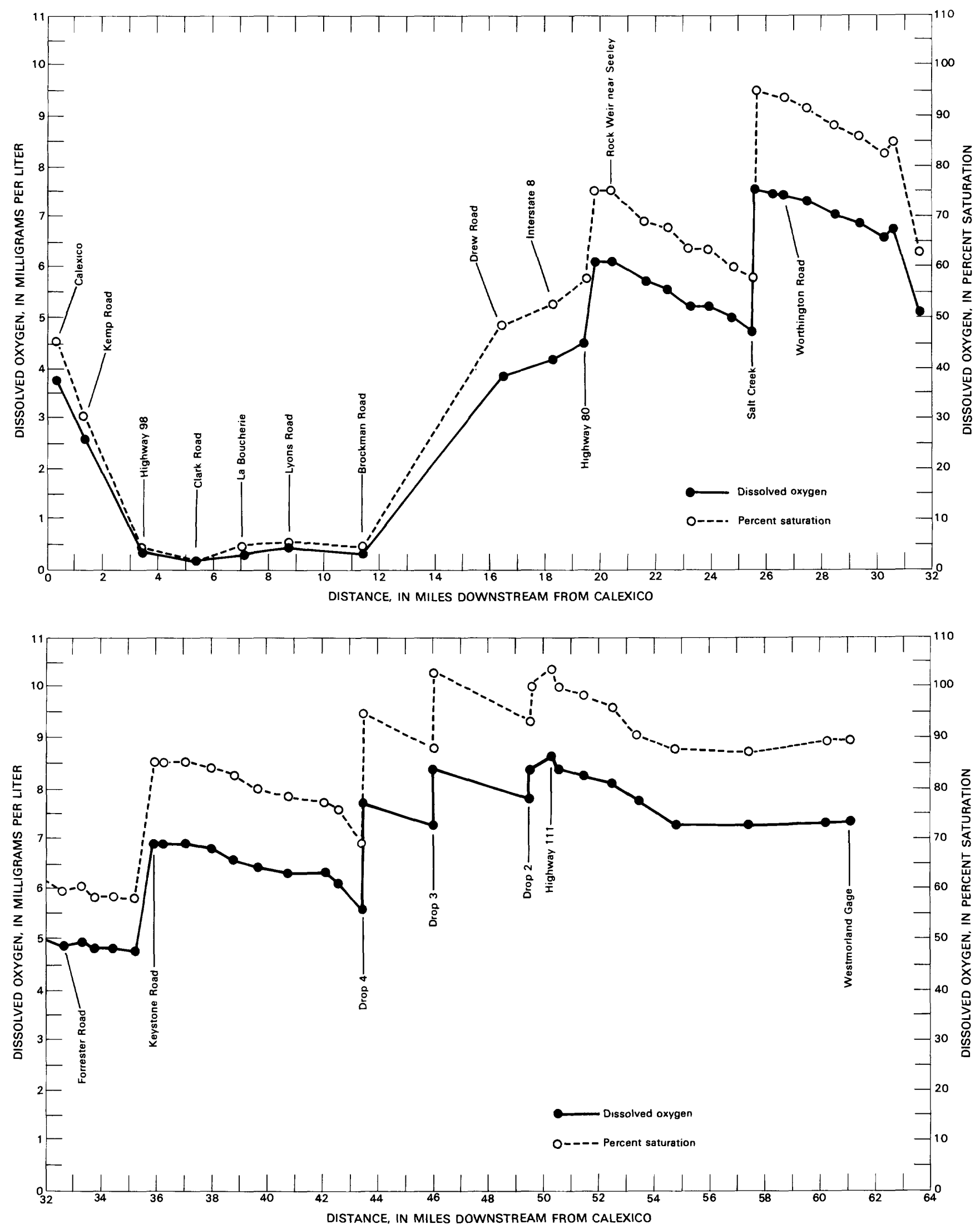

Figure 10. Dissolved-oxygen concentration and percent saturation in the New River from Calexico to the Westmorland gage, September 26-30, 1977. 
Table 7. Field measurements of selected physical and chemical characteristics at sites on the New River, September 26-30, 1977

[Site number is miles downstream from the international boundary at Calexico]

\begin{tabular}{lcccccc}
\hline Date, time & $\begin{array}{c}\text { Site } \\
\text { No. }\end{array}$ & $\begin{array}{c}\text { Specific } \\
\text { conductance } \\
\text { (mmho/cm } \\
\left.\text { at } 25^{\circ} \mathrm{C}\right)\end{array}$ & $\mathrm{pH}$ & $\begin{array}{c}\text { Water } \\
\text { temper- } \\
\text { ature } \\
\left({ }^{\circ} \mathrm{C}\right)\end{array}$ & $\begin{array}{c}\text { Dis- } \\
\text { solved } \\
\text { oxygen } \\
\text { (mg/L) }\end{array}$ & Remarks \\
\hline $9-30$ at 1020 & 0 & 7,280 & 8.0 & 24.5 & 3.7 & At Calexico. \\
$9-30$ at 0955 & 1.5 & 6,890 & 8.0 & 24.1 & 2.6 & At Kemp Road. \\
$9-30$ at 0945 & 3.5 & 6,660 & 8.0 & 24.8 & 0.3 & At Highway 98. \\
$9-30$ at 0930 & 5.5 & 6,300 & 8.0 & 24.6 & 0.2 & At Clark Road. \\
$9-30$ at 0905 & 7.2 & 6,050 & 8.0 & 24.7 & 0.3 & At La Boucherie. \\
$9-30$ at 0840 & 8.8 & 6,470 & 7.9 & 24.8 & 0.4 & At Lyons Road. \\
$9-30$ at 0820 & 11.4 & 6,620 & 7.8 & 24.8 & 0.3 & At Bockman Road. \\
$9-29$ at 1610 & 16.4 & 6,490 & 8.2 & 27.2 & 3.8 & At Drew Road. \\
$9-29$ at 1555 & 18.4 & 6,470 & 8.0 & 27.0 & 4.2 & At Interstate 8. \\
$9-29$ at 1520 & 19.5 & 6,440 & 8.0 & 26.7 & 4.5 & At Highway 80. \\
& & & & & & \\
$9-29$ at 1120 & 19.8 & 6,710 & 7.9 & 25.5 & 6.1 & \\
$9-29$ at 1110 & 20.4 & 6,570 & 7.8 & 25.7 & 6.1 & \\
$9-29$ at 1045 & 21.7 & 6,440 & 7.8 & 25.2 & 5.7 & \\
$9-29$ at 1035 & 22.5 & 6,630 & 7.8 & 25.2 & 5.5 & \\
$9-29$ at 1020 & 23.2 & 6,660 & 7.8 & 25.0 & 5.2 & \\
$9-29$ at 1000 & 24.0 & 6,710 & 7.8 & 24.8 & 5.2 & \\
$9-29$ at 0945 & 24.9 & 6,680 & 7.8 & 24.7 & 5.0 & \\
$9-29$ at 0930 & 25.6 & 6,610 & 7.8 & 24.8 & 4.7 & \\
$9-28$ at 1655 & 25.6 & 6,930 & 8.0 & 27.5 & 7.5 & At Worthington Road. \\
$9-28$ at 1632 & 26.4 & 6,830 & 8.0 & 27.2 & 7.5 & \\
$9-28$ at 1626 & 26.7 & 6,850 & 8.0 & 27.2 & 7.4 & \\
$9-28$ at 1610 & 27.6 & 6,850 & 7.9 & 27.2 & 7.3 & \\
$9-28$ at 1552 & 28.6 & 6,840 & 7.9 & 27.2 & 7.0 & \\
$9-28$ at 1540 & 29.4 & 6,750 & 7.9 & 27.2 & 6.8 & \\
$9-28$ at 1526 & 30.2 & 6,700 & 7.9 & 27.1 & 6.5 & \\
$9-28$ at 1518 & 30.7 & 6,760 & 7.9 & 27.3 & 6.7 & \\
& & & & & &
\end{tabular}

For example, the concentrations at Calexico were more than two times that at Westmorland with COD's of 170 versus $73 \mathrm{mg} / \mathrm{L}$, BOD's of 20 versus $8.4 \mathrm{mg} / \mathrm{L}$, and TOC's of 17 versus $8.6 \mathrm{mg} / \mathrm{L}$. These numbers show that there is sufficient organic matter in the water at the Calexico gage to deplete the available dissolved oxygen. With BOD reaction rate of about 25 percent per day, it would take $2 \frac{1}{2}$ days, which is the approximate traveltime between Calexico and Westmorland, to reduce the demand to one-half its original value. No discernible pattern developed from the remaining data other than the generalization already stated. For example, at Kemp Road, $1.5 \mathrm{mi}$ downstream, the concentrations were $130 \mathrm{mg} / \mathrm{L}$ for COD, $6.4 \mathrm{mg} / \mathrm{L}$ for BOD, and $12 \mathrm{mg} / \mathrm{L}$ for TOC. Because the water observed at Kemp Road did not necessarily cross the international boundary with the same quality as the water sampled at the Calexico gage (due to time of travel and variation in water quality at the international boundary), these lower concentrations cannot be attributed entirely to aerobic oxidation. The lower demands may be due in part to dilution and settling out of particulate matter.

The profile of May 10, 1978, (fig. 17) also showed that the concentration of organic material decreased downstream. COD and TOC were $300 \mathrm{mg} / \mathrm{L}$ and 19.7 $\mathrm{mg} / \mathrm{L}$ at the Calexico gage compared with $75 \mathrm{mg} / \mathrm{L}$ and $12.8 \mathrm{mg} / \mathrm{L}$, respectively, at Lack Road $60.2 \mathrm{mi}$ downstream. The accumulation of settleable solids from these high organic loads results in sludge deposits that in the upper reaches of the New River undergo anaerobic fermentation. The products of that oxidation leach into the flowing water and exert a demand on the dissolved oxygen assets of the river. According to Velz (1970, p. 162), "When the sludge deposit is large in relation to the overflowing streams, dissolved oxygen may be completely exhausted and the gas bubbles may rise and break at the water surface, releasing hydrogen sulfide and other gases to the atmosphere." These conditions have been observed in the reach from Calexico to Drew Road.

\section{Diel Measurements}

The concentrations and movement of organic material were also demonstrated during the two diel studies. The diel study of October 17-18, 1977, showed the movement of a slug discharge from Calexico to Highway 80, $19.5 \mathrm{mi}$ and 17 to 19 hours downstream. At the Calexico 
Table 7. Field measurements of selected physical and chemical characteristics at sites on the New River, September 26-30, 1977 -Continued

\begin{tabular}{|c|c|c|c|c|c|c|c|}
\hline Date, & , time & $\begin{array}{l}\text { Site } \\
\text { No. }\end{array}$ & $\begin{array}{l}\text { Specific } \\
\text { conductance } \\
(\text { mmho } / \mathrm{cm} \\
\left.\text { at } 25^{\circ} \mathrm{C}\right)\end{array}$ & $\mathrm{pH}$ & $\begin{array}{l}\text { Water } \\
\text { temper- } \\
\text { ature } \\
\left({ }^{\circ} \mathrm{C}\right)\end{array}$ & $\begin{array}{l}\text { Dis- } \\
\text { solved } \\
\text { oxygen } \\
(\mathrm{mg} / \mathrm{L})\end{array}$ & Remarks \\
\hline $\begin{array}{ll}9-28 & \text { a } \\
9-28 & \text { a } \\
9-28 & \text { a } \\
9-28 & \text { a } \\
9-28 & \text { a }\end{array}$ & $\begin{array}{ll}\text { at } & 1045 \\
\text { at } & 1010 \\
\text { at } & 0928 \\
\text { at } & 0914 \\
\text { at } & 0903\end{array}$ & $\begin{array}{l}31.7 \\
32.9 \\
33.4 \\
34.0 \\
34.6\end{array}$ & $\begin{array}{l}6,240 \\
6,290 \\
6,320 \\
6,290 \\
6,390\end{array}$ & $\begin{array}{l}7.8 \\
7.8 \\
7.8 \\
7.8 \\
7.8\end{array}$ & $\begin{array}{l}25.1 \\
25.0 \\
24.9 \\
24.8 \\
24.9\end{array}$ & $\begin{array}{l}5.1 \\
4.9 \\
5.0 \\
4.8 \\
4.8\end{array}$ & At Forrester Road. \\
\hline $\begin{array}{ll}9-28 & \text { a } \\
9-27 & \text { a } \\
9-27 & \text { a } \\
9-27 & \text { a } \\
9-27 & \text { a }\end{array}$ & $\begin{array}{ll}\text { at } & 0853 \\
\text { at } & 1615 \\
\text { at } & 1610 \\
\text { at } & 1600 \\
\text { at } & 1543\end{array}$ & $\begin{array}{l}35.2 \\
36.1 \\
36.4 \\
37.2 \\
38.1\end{array}$ & $\begin{array}{l}6,400 \\
6,620 \\
6,540 \\
6,590 \\
6,590\end{array}$ & $\begin{array}{l}7.8 \\
7.8 \\
7.8 \\
7.8 \\
7.8\end{array}$ & $\begin{array}{l}24.9 \\
26.5 \\
26.8 \\
26.9 \\
27.0\end{array}$ & $\begin{array}{l}4.7 \\
6.8 \\
6.8 \\
6.8 \\
6.7\end{array}$ & At Keystone Road. \\
\hline $\begin{array}{ll}9-27 & \text { a } \\
9-27 & \text { a } \\
9-27 & \text { a } \\
9-27 & \text { a } \\
9-27 & \text { a }\end{array}$ & $\begin{array}{ll}\text { at } & 1521 \\
\text { at } & 1510 \\
\text { at } & 1455 \\
\text { at } & 1443 \\
\text { at } & 1435\end{array}$ & $\begin{array}{l}39.0 \\
39.8 \\
41.0 \\
42.2 \\
42.9\end{array}$ & $\begin{array}{l}6,340 \\
6,340 \\
6,340 \\
6,410 \\
6,480\end{array}$ & $\begin{array}{l}7.8 \\
7.8 \\
7.8 \\
7.8 \\
7.8\end{array}$ & $\begin{array}{l}26.9 \\
26.9 \\
26.8 \\
26.5 \\
26.3\end{array}$ & $\begin{array}{l}6.5 \\
6.4 \\
6.2 \\
6.2 \\
6.1\end{array}$ & At Highway 78 and 99 \\
\hline $\begin{array}{ll}9-27 & \text { a } \\
9-27 & \text { a } \\
9-27 & \text { a } \\
9-27 & \text { a } \\
9-27 & \text { a }\end{array}$ & $\begin{array}{ll}\text { at } & 1345 \\
\text { at } & 1345 \\
\text { at } & 1155 \\
\text { at } & 1155 \\
\text { at } & 1130\end{array}$ & $\begin{array}{l}43.6 \\
43.6 \\
46.2 \\
46.2 \\
49.8\end{array}$ & $\begin{array}{l}6,250 \\
6,240 \\
6,300 \\
6,330 \\
6,530\end{array}$ & $\begin{array}{l}7.8 \\
7.9 \\
7.8 \\
7.9 \\
7.9\end{array}$ & $\begin{array}{l}26.1 \\
26.1 \\
25.3 \\
25.3 \\
25.0\end{array}$ & $\begin{array}{l}5.6 \\
7.7 \\
7.2 \\
8.4 \\
7.7\end{array}$ & $\begin{array}{l}\text { Above Drop } 4 . \\
\text { Below Drop } 4 . \\
\text { Above Drop } 3 . \\
\text { Below Drop } 3 . \\
\text { Above Drop } 2 .\end{array}$ \\
\hline $\begin{array}{ll}9-27 & a \\
9-27 & a \\
9-27 & a \\
9-27 & a \\
9-27 & a\end{array}$ & $\begin{array}{ll}\text { at } 1130 \\
\text { at } 1042 \\
\text { at } 1034 \\
\text { at } 1017 \\
\text { at } 1000\end{array}$ & $\begin{array}{l}49.8 \\
50.3 \\
50.6 \\
51.8 \\
52.7\end{array}$ & $\begin{array}{l}6,570 \\
6,510 \\
6,490 \\
6,470 \\
6,230\end{array}$ & $\begin{array}{l}7.9 \\
7.9 \\
7.9 \\
7.9 \\
7.9\end{array}$ & $\begin{array}{l}25.0 \\
24.8 \\
24.6 \\
24.8 \\
24.3\end{array}$ & $\begin{array}{l}8.3 \\
8.6 \\
8.4 \\
8.2 \\
8.1\end{array}$ & $\begin{array}{l}\text { Below Drop } 2 . \\
\text { At Highway } 111 .\end{array}$ \\
\hline $\begin{array}{ll}9-27 & a \\
9-26 & a \\
9-26 & a \\
9-26 & a \\
9-26 & a\end{array}$ & $\begin{array}{l}\text { at } 0945 \\
\text { at } 1700 \\
\text { at } 1600 \\
\text { at } 1500 \\
\text { at } 1430\end{array}$ & $\begin{array}{l}53.6 \\
55.0 \\
57.3 \\
60.2 \\
61.2\end{array}$ & $\begin{array}{l}6,290 \\
6,020 \\
5,700 \\
5,620 \\
5,600\end{array}$ & $\begin{array}{l}7.9 \\
8.0 \\
8.0 \\
8.1 \\
8.1\end{array}$ & $\begin{array}{l}24.2 \\
26.0 \\
26.0 \\
26.0 \\
26.0\end{array}$ & $\begin{array}{l}7.7 \\
7.2 \\
7.2 \\
7.3 \\
7.3\end{array}$ & $\begin{array}{l}\text { At Gentry Road. } \\
\text { At Lack Road. } \\
\text { At Westmorland gage. }\end{array}$ \\
\hline
\end{tabular}

gage on October 17, at $\mathbf{i} 300$ hours, the DO concentration was $3 \mathrm{mg} / \mathrm{L}$ and the COD, TOC, and DOC were 120 , 14.4 , and $9.4 \mathrm{mg} / \mathrm{L}$ respectively (fig. 11). Five hours later, the DO concentration had decreased to zero and the COD, TOC, and DOC had increased to 480,20 , and $15 \mathrm{mg} / \mathrm{L}$, respectively. These increases indicate the passage of a slug discharge. Downstream at Highway 80, the initial BOD for the diel study was $5.4 \mathrm{mg} / \mathrm{L}$. At 1400 hours on October 18 , the slug discharge that was detected at the Calexico gage arrived at Highway 80, sending the BOD up to $18.4 \mathrm{mg} / \mathrm{L}$ as the DO concentration decreased from $2 \mathrm{mg} / \mathrm{L}$ to zero.

The sample collected at the Highway 80 crossing at 1600 hours had a COD of $93 \mathrm{mg} / \mathrm{L}$, TOC concentration of $11.2 \mathrm{mg} / \mathrm{L}$, and a DOC concentration of $7.5 \mathrm{mg} / \mathrm{L}$. At Keystone Road 15 hours later, the approximate traveltime from Highway 80, the COD had decreased to 77 $\mathrm{mg} / \mathrm{L}$, and the TOC and DOC concentrations had decreased to $9.8 \mathrm{mg} / \mathrm{L}$ and $6.8 \mathrm{mg} / \mathrm{L}$, respectively. The other three sites, Keystone, Drop 4, and Westmorland, showed a gradual decrease in concentration between sites in downstream order.

Loads

To determine the loads of selected constituents in the New River at Calexico, a program of composite sampling was devised. Load is the weight per unit time, usually expressed in tons per day, of a substance passing a point. It takes into account the volume of water and the concentration of the substance. By collecting and compositing a water sample every 15 minutes for 12 hours, the influence of the periodic slug discharges would be mediated and the average condition of the water at the Calexico gage could be determined. A 12-hour set of composites was collected in each of the months from July to December 1978, with the exception of October. For October, samples were collected for eight consecutive 12 hour periods. At the end of each 12 hours, samples for 


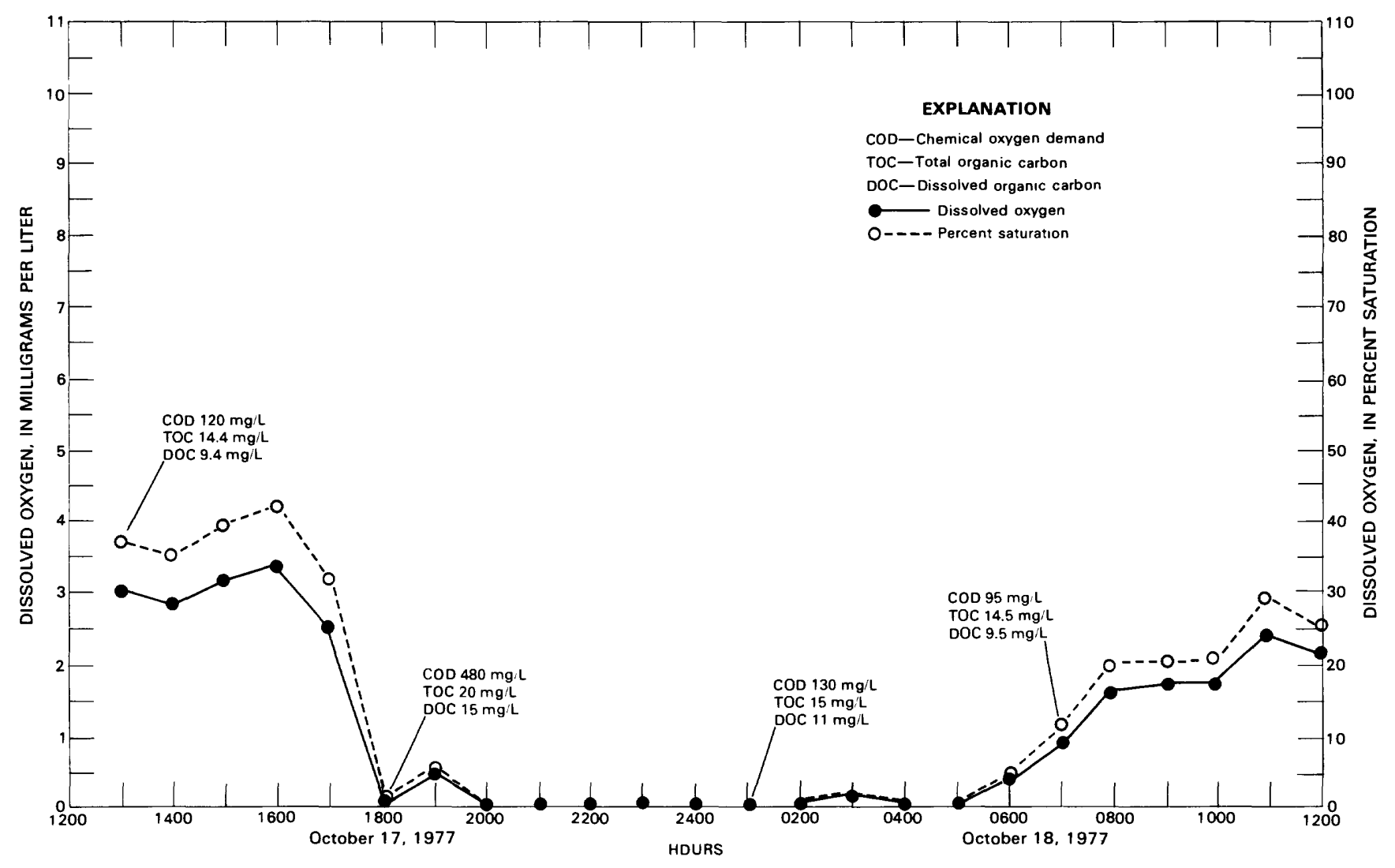

Figure 11. Dissolved-oxygen concentration, percent saturation, and selected chemical constituents, New River at Calexico, October 17-18, 1977.

laboratory analysis were split from the composited water. Table 10 presents the loads of selected constituents for the composite sampling program. The highest BOD load, in tons per day, was 133 on September 26; the lowest was 9.5 on July 26,1978 .

\section{Biota as Indicators}

Thus far, the emphasis of this report has been on identifying the magnitude and occurrence of chemical and physical constituents in the New River, especially in the flow at the international boundary, and their effects on downstream water quality. This information provides the basis for a better understanding of the ongoing chemical processes, showing cause and effect. With that understanding, remedial steps can be formulated if necessary. Sampling of most chemical constituents represents conditions at a single point in time. Even when the program involves intensive around-the-clock sampling, it can never be all-inclusive as an indicator of the suitability of the environment to support aquatic life. Perhaps, then, a good test of a river's health is the identification and quantification of its biota.
Stream biota must withstand extremes in water quality (slug flows, anaerobic conditions, and high and low temperatures) as well as adapt to average conditions. Because environmental conditions affect the kinds of organisms that can inhabit a specific reach of a river, the types of organisms present provide an index of the environmental conditions of that reach. The presence of a species signifies that the minimal conditions necessary to sustain the life of that organism are being met. The absence of a species or even a group of organisms cannot, however, be taken as an indication that the conditions necessary to support that group are not present. It is entirely possible that there has been no opportunity for that observed group to become established or introduced to the reach.

The health index of a stream is best determined by observing the population and community of the biota rather than the presence or absence of an individual species. A healthy stream will have a high diversity of organisms. The high diversity leads to a stable community in that the risk is spread out through a variety of organisms. An equilibrium between the environment and the biota can be more harmoniously maintained when the diversity is high. When subjected to a pollutional stress, 


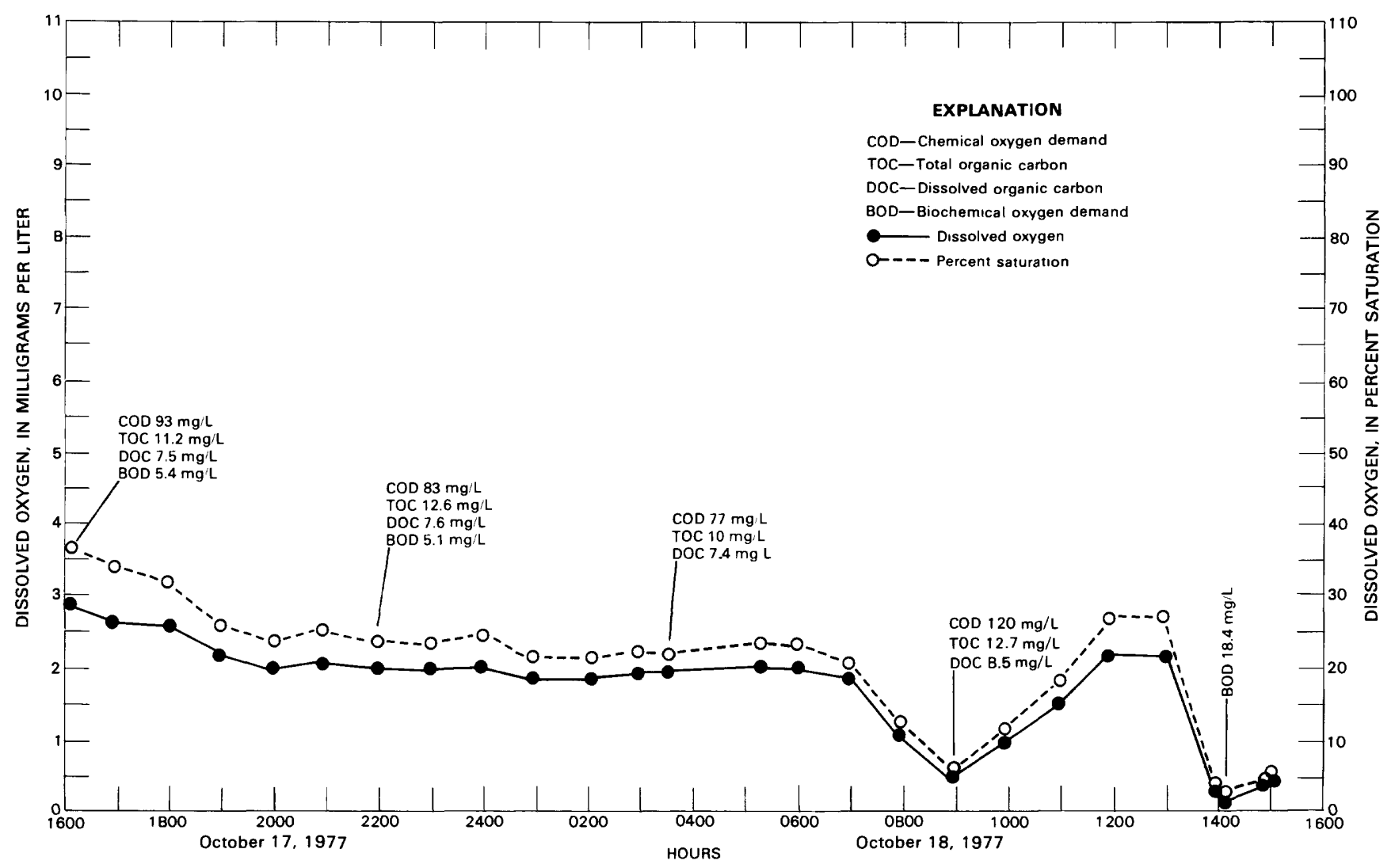

Figure 12. Dissolved-oxygen concentration, percent saturation, and selected chemical constituents, New River at Highway 80, October 17-18, 1977.

the sensitive species or groups are eliminated and the tolerant survive. The diversity decreases but the number of organisms per species usually increases. As the pollutional stress becomes too severe, the number of individuals per species is reduced, as is the number of species, until no animal life exists.

\section{Benthic Invertebrates}

Benthic invertebrates are, as the name indicates, those animals that lack a backbone and inhabit the bottom of streams and lakes. The U.S. Geological Survey has adopted the Standard Sieve No. 70 (210-micrometer mesh openings) as the retention criterion for the collection of benthic invertebrate organisms from streambeds. Samples for the analysis of benthic invertebrates were collected three times yearly at the following New River sites:

At international boundary at Calexico

At Lyons Crossing

At Keystone Road

At Westmorland gage.

Control samples were collected at the Alamo River at out- let near Niland for comparison to show the effects of the slug discharges on the diversity of the biota.

The cumulative effect of the slug discharges on the number and diversity of organisms is shown in figure 18 and table 11 . The water crossing the international boundary at Calexico seems of such poor quality that very little animal life can exist in the reach between Calexico and Highway 80. The data show that the first two sites, Calexico gage and Lyons Crossing, have similar environmental conditions. For each of the three sets of samples collected, no more than three species and as few as one organism were present. The concepts of community structure and population are not applicable to these two sites because the diversity and population are minimal.

Downstream at Keystone Road (site 36.1), the population and community structure changed. The highest numbers of organisms per multiplate sampler were obtained at this site, 9,150 on April 25 and 1,120 on October 25 . The diversity at the genus level was greater at Keystone than at the first two sites. On April 25, there were 8,768 organisms per multiplate sampler of the Nais pardalis, which is an aquatic earthworm associated with organically rich substrates and often with water having a low DO concentration. The overabundance of this one 

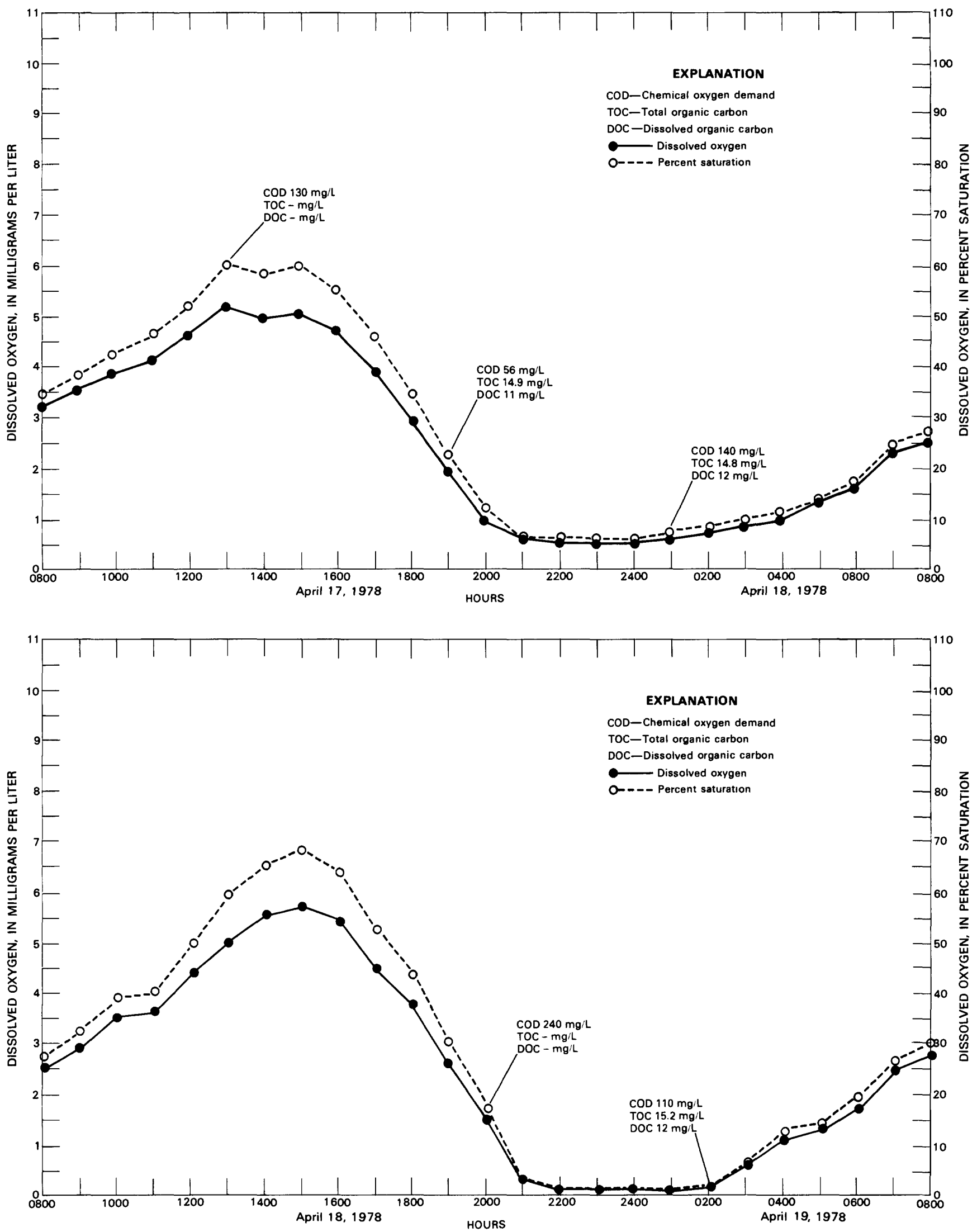

Figure 13. Dissolved-oxygen concentration, percent saturation, and selected chemical constituents, New River at Calexico, April 17-19, 1978. 


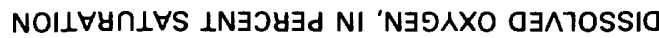

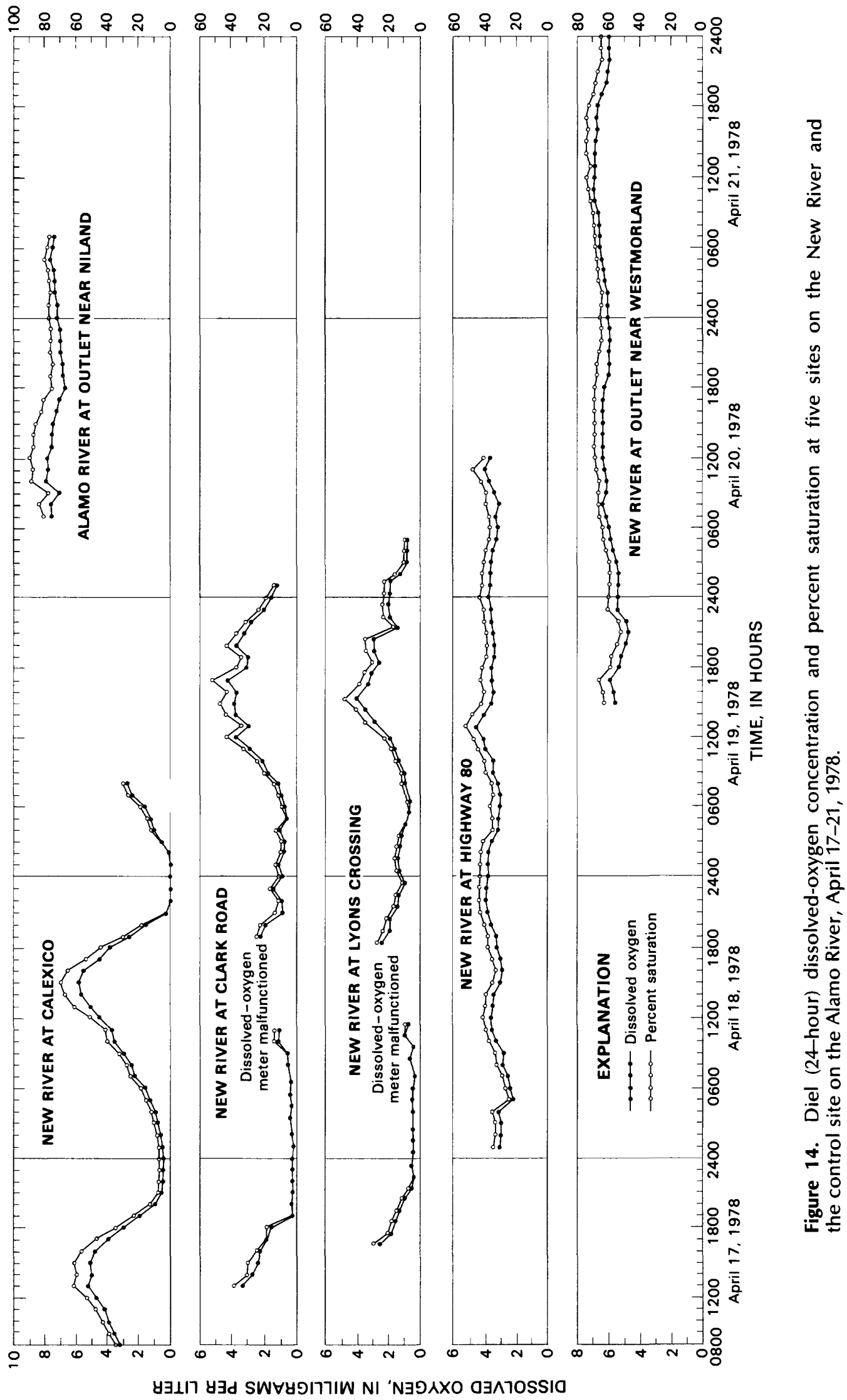



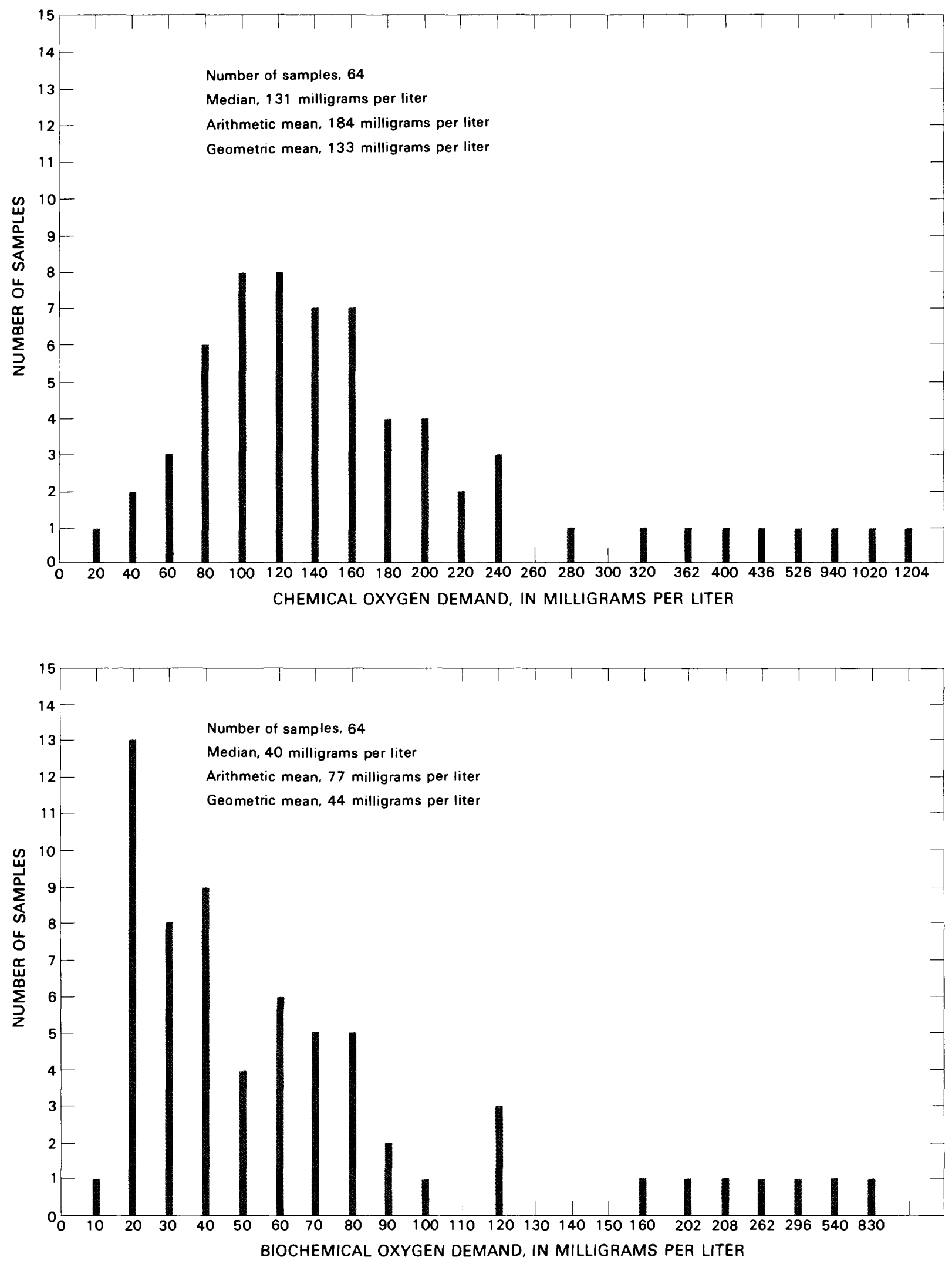

Figure 15. Distribution of concentrations of chemical oxygen demand and biochemical oxygen demand, New River at Calexico, July 1976 to March 1978. 
Table 8. Time-of-travel data for selected sites on the New River, February 6-9, and June 19-22, 1978

\begin{tabular}{|c|c|c|c|c|c|c|c|c|}
\hline \multirow{2}{*}{ Site } & \multirow{2}{*}{$\begin{array}{l}\text { Cumula- } \\
\text { tive } \\
\text { miles }\end{array}$} & \multicolumn{3}{|c|}{$\begin{array}{c}\text { Arrival of leading edge } \\
\text { of dye }\end{array}$} & \multicolumn{3}{|c|}{$\begin{array}{l}\text { Arrival of peak } \\
\text { concentration of dye }\end{array}$} & \multirow{3}{*}{$\begin{array}{l}\text { Time and date of } \\
\text { indicated percentage } \\
\text { of peak dye concen- } \\
\text { tration on recession }\end{array}$} \\
\hline & & Time, date & $\begin{array}{l}\text { Cum } \\
\text { hours }\end{array}$ & $\begin{array}{l}\text { ive } \\
\text { inutes }\end{array}$ & Time, date & $\begin{array}{r}\text { Cum } \\
\text { hours }\end{array}$ & $\begin{array}{l}\text { ive } \\
\text { nutes }\end{array}$ & \\
\hline \multicolumn{8}{|c|}{ February 6-9, 1978} & \\
\hline Calexico & 0 & $\begin{array}{c}1200,2-6 \\
\text { (dye injected) }\end{array}$ & - & - & - & $=$ & - & $-\quad-$ \\
\hline Clark Road & 5.5 & $1700,2-6$ & 5 & 0 & $1740,2-6$ & 5 & 40 & $\begin{array}{l}5 \text { percent of peak } \\
1840,2-6\end{array}$ \\
\hline Lyons Crossing & 8.8 & $2010,2-6$ & 8 & 10 & $2120,2-6$ & 9 & 20 & $\begin{array}{l}5 \text { percent of peak } \\
2250,2-6\end{array}$ \\
\hline Drew Road & 16.4 & $0315,2-7$ & 15 & 15 & $0435,2-7$ & 16 & 35 & \\
\hline Highway 80 & 19.5 & $05--, 2-7$ & 17 & 0 & $0730,2-7$ & 19 & 30 & $\begin{array}{l}5 \text { percent of peak } \\
0945,2-7\end{array}$ \\
\hline $\begin{array}{l}\text { Worthington } \\
\text { Road }\end{array}$ & 26.4 & $1130,2-7$ & 23 & 30 & $1325,2-7$ & 25 & 25 & $\begin{array}{l}10 \text { percent of peak } \\
1540,2-7\end{array}$ \\
\hline $\begin{array}{l}\text { Keystone } \\
\text { Road }\end{array}$ & 36.1 & $2000,2-7$ & 32 & 00 & $2210,2-7$ & 34 & 10 & $\begin{array}{l}10 \text { percent of peak } \\
0200,2-8\end{array}$ \\
\hline Drop 4 & 43.6 & $0300,2-8$ & 39 & 00 & $0530,2-8$ & 41 & 30 & $\begin{array}{l}15 \text { percent of peak } \\
0930,2-8\end{array}$ \\
\hline $\begin{array}{l}\text { Rutherford } \\
\text { Road }\end{array}$ & 50.3 & $0900,2-8$ & 45 & 00 & $1130,2-8$ & 47 & 30 & $\begin{array}{l}10 \text { percent of peak } \\
1600,2-8\end{array}$ \\
\hline Gentry Road & 57.5 & $1530,2-8$ & 51 & 30 & $1750,2-8$ & 53 & 50 & $\begin{array}{l}10 \text { percent of peak } \\
2200,2-8\end{array}$ \\
\hline $\begin{array}{l}\text { Westmorland } \\
\text { Gage }\end{array}$ & 61.2 & $1830,2-8$ & 54 & 30 & $2115,2-8$ & 57 & 15 & $\begin{array}{l}10 \text { percent of peak } \\
0230,2-9\end{array}$ \\
\hline \multicolumn{9}{|c|}{ June $19-22,1978$} \\
\hline Calexico & 0 & $\begin{array}{c}1200,6-19 \\
\text { (dye injected) }\end{array}$ & - & - & - & - & - & $-\quad-$ \\
\hline Clark Road & 5.5 & $1710,6-19$ & 5 & 10 & $1800,6-19$ & 6 & 0 & $\begin{array}{l}5 \text { percent of peak } \\
1905,6-19\end{array}$ \\
\hline Lyons Crossing & 8.8 & $2040,6-19$ & 8 & 40 & $2155,6-19$ & 9 & 55 & $\begin{array}{l}5 \text { percent of peak } \\
2325,6-19\end{array}$ \\
\hline Drew Road & 16.4 & $0415,6-20$ & 16 & 15 & $0535,6-19$ & 17 & 35 & $\begin{array}{l}5 \text { percent of peak } \\
0835,6-20\end{array}$ \\
\hline Highway 80 & 19.5 & $0700,6-20$ & 19 & 0 & $0830,6-20$ & 20 & 30 & $\begin{array}{l}5 \text { percent of peak } \\
1050,6-20\end{array}$ \\
\hline $\begin{array}{l}\text { Worthington } \\
\text { Road }\end{array}$ & 26.4 & $\begin{array}{l}1400,6-20 \\
\text { (dye present in } \\
\text { first sample) }\end{array}$ & F26 & - & $1445,6-20$ & 26 & 45 & $\begin{array}{l}5 \text { percent of peak } \\
1900,6-20\end{array}$ \\
\hline $\begin{array}{l}\text { Keystone } \\
\text { Road }\end{array}$ & 36.1 & $2120,6-20$ & 33 & 20 & $2300,6-20$ & 35 & 0 & $\begin{array}{l}13 \text { percent of peak } \\
0200,6-21\end{array}$ \\
\hline Drop 4 & 43.6 & $0420,6-21$ & 40 & 20 & $0640,6-21$ & 42 & 40 & $\begin{array}{l}10 \text { percent of peak } \\
1010,6-21\end{array}$ \\
\hline $\begin{array}{l}\text { Rutherford } \\
\quad \text { Road }\end{array}$ & 50.3 & $0930,6-21$ & 45 & 30 & $1215,6-21$ & 48 & 15 & $\begin{array}{l}10 \text { percent of peak } \\
1620,6-21\end{array}$ \\
\hline Gentry Road & 57.5 & $1545,6-21$ & 51 & 45 & $1830,6-21$ & 54 & 30 & $\begin{array}{l}10 \text { percent of peak } \\
0005,6-22\end{array}$ \\
\hline $\begin{array}{l}\text { Westmorland } \\
\text { Gage }\end{array}$ & 61.2 & $21900,6-21$ & Z55 & - & $2145,6-21$ & 57 & 45 & $\begin{array}{l}10 \text { percent of peak } \\
0330,6-22\end{array}$ \\
\hline
\end{tabular}



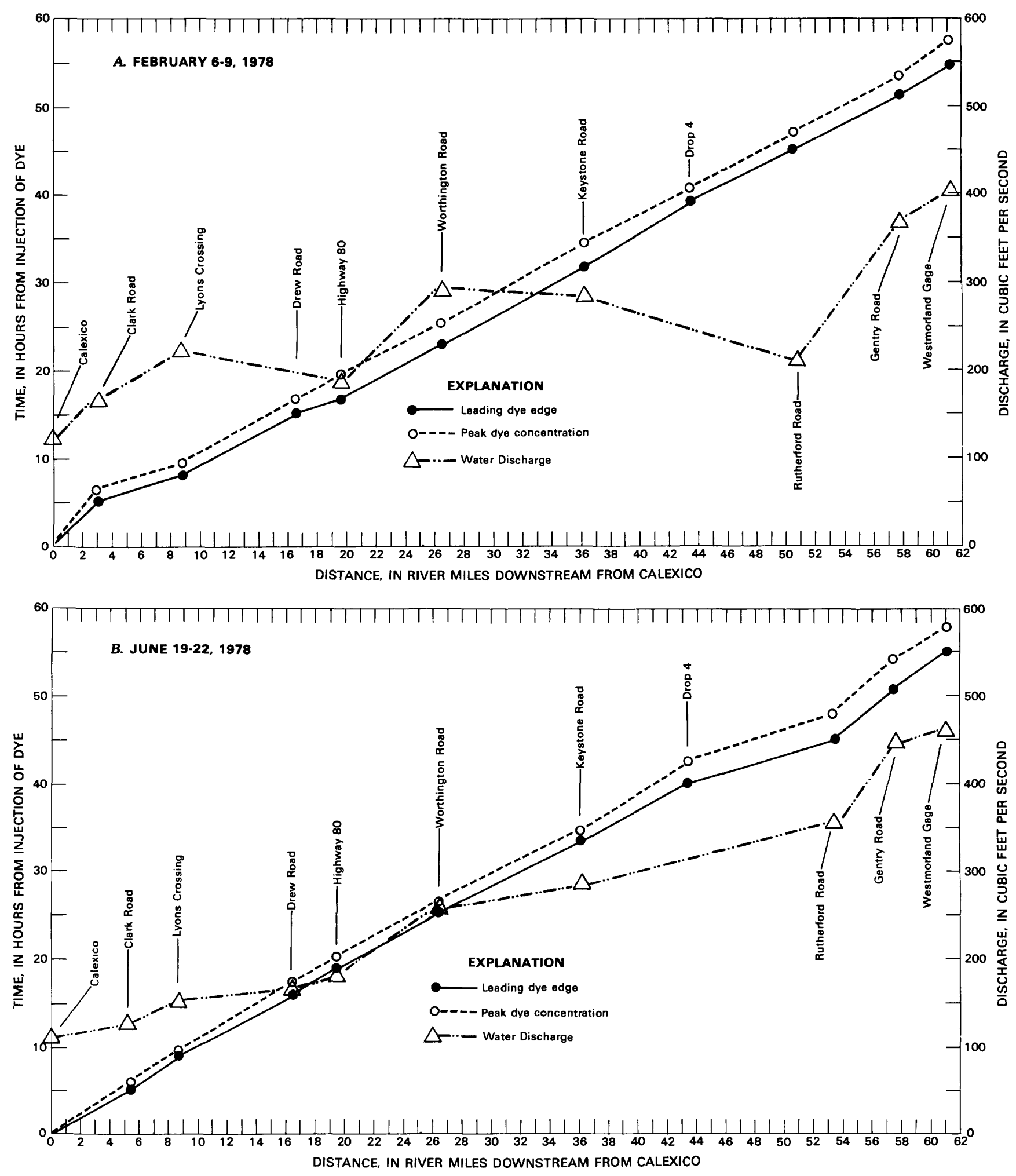

Figure 16. Time of travel of dye and discharge in the New River from Calexico to Westmorland gage, February 6-9 and June 19-22, 1978.

species is indicative of pollutional stress but, in this case, shows that recovery or self-purification of the river is occurring with respect to the conditions observed at Calexico and Lyons Crossing. The other two samples also have one or two dominant species that are present in disproportionately high numbers. For the October sample, the 
Table 9. Determinations of selected physical, chemical, and biological characteristics at sites on the New River, September 26-30, 1977

[Site number is number of miles downstream from international boundary at Calexico. BOD, biochemical oxygen demand; COD, chemical oxygen demand; DOC, dissolved organic carbon; Ec, specific conductance; FC, fecal coliform; FS, fecal streptococci; TOC, total organic carbon]

\begin{tabular}{cccccccc}
\hline $\begin{array}{c}\text { Site } \\
\text { No. }\end{array}$ & $\begin{array}{c}\text { EC } \\
(\mu \mathrm{mho} / \mathrm{cm} \\
\left.\text { at } 25^{\circ} \mathrm{C}\right)\end{array}$ & $\begin{array}{c}\text { COD } \\
(\mathrm{mg} / \mathrm{L})\end{array}$ & $\begin{array}{c}\text { TOC } \\
(\mathrm{mg} / \mathrm{L})\end{array}$ & $\begin{array}{c}\text { DOC } \\
(\mathrm{mg} / \mathrm{L})\end{array}$ & $\begin{array}{c}\text { BOD } \\
(\mathrm{mg} / \mathrm{L})\end{array}$ & $\begin{array}{c}\text { FC } \\
(\mathrm{col} / \\
(100 \mathrm{~mL})\end{array}$ & $\begin{array}{c}\text { FS } \\
(\mathrm{col} /\end{array}$ \\
\hline
\end{tabular}

\begin{tabular}{rrrrrrrr}
0 & 7280 & 170 & 17 & 15 & 20 & 460,000 & 240,000 \\
1.5 & 6890 & 130 & 12 & 9.6 & 6.4 & 620,000 & 120,000 \\
7.2 & 6050 & 79 & 13 & 8.6 & 15 & -- & -- \\
8.8 & 6470 & 79 & 12 & 7.9 & 7.6 & 780,000 & 62,000 \\
11.4 & 6620 & 92 & 14 & 9.0 & 12 & $2,800,000$ & 180,000 \\
16.4 & 6490 & 120 & 12 & 7.6 & 8.4 & -- & -- \\
19.8 & 6710 & 110 & 7.3 & 7.3 & 6.0 & 180,000 & 5,000 \\
24.0 & 6710 & 100 & -- & -- & 8.0 & 250,000 & 30,000 \\
26.7 & 6850 & 100 & 11 & 8.4 & 6.8 & -- & -- \\
27.6 & 6850 & 69 & -- & -- & 8.7 & -- & - \\
32.9 & 6290 & 68 & -- & -- & 14 & 980,000 & - \\
36.1 & 6620 & 81 & -- & 7.4 & -- & 3,700 & 13,000 \\
39.0 & 6340 & 83 & -- & -- & 7.6 & 3,000 & $<1,000$ \\
43.6 & 6240 & 110 & 9.4 & 8.0 & 6.4 & 8,000 & $<1,000$ \\
50.3 & 6510 & -- & -- & -- & -- & 110,000 & 5,300 \\
52.7 & 6230 & 100 & 9.1 & 7.3 & 5.6 & 17,000 & 6,000 \\
55.0 & 6020 & 99 & 11 & 9.4 & 10 & 24,000 & 12,000 \\
57.5 & 5700 & 84 & -- & -- & 8.0 & 13,300 & 11,200 \\
60.2 & 5620 & 77 & 9.2 & 8.1 & 7.6 & 11,000 & 11,000 \\
61.2 & 5600 & 73 & 8.6 & 6.9 & 8.4 & 14,700 & 1400 \\
\hline
\end{tabular}

${ }^{1}$ Nonideal count.

genus Chironomus (one of the midges) dominated, with 784 organisms out of a total of 1,120. In June, another one of the midges, Goeldichironomus holoprasinus, and a tubificid, Limnodrilus hoffmeisteri, dominated, with 378 and 208 organisms, respectively, out of 619 . There were 5 other genera present in this sample and 14 other genera present in the October sample.

At the Westmorland gage (site 61.2), the community was not as large as at Keystone but the diversity was greater. The June sample had the largest community, 777 organisms, and the greatest number of species, 16. Although two species were dominant, they were not as disproportionate in number as at Keystone. The diversity at the family level was also greater than at the other sites, indicating that organisms of varying requirements were able to survive. Diversities at the phylum and class taxonomic levels for the June sample were the highest of the New River sites but less than what would be found in a healthy river. Organisms from a variety of phyla, including Nematoda, Annelida. Arthropoda, and Coelenterata (colonial form) were present. The data show that the water quality was continuing to improve when compared to the Keystone site.

Diversity was greatest at the control site, Alamo River at outlet near Niland. For the June sample, 551 organisms were distributed among 26 species. There were two codominants present, Rheotanytarus (a midge), with 142 organisms, and Boccardia (a polychaete which are mostly marine), with 181 organisms. The April sample did not have as great a diversity as the June sample because the April sample was dominated by one genus, Cricotopus, with 376 organisms out of a sample total of 529. The September sample seems to be anomalous with not only the April and June samples but also with the Westmorland gage data.

\section{Phytoplankton}

Phytoplankton, which means literally wandering plants but usually refers to the algae that drift in streams or lakes, are also an indication of environmental conditions. In streams, the phytoplankton are not as good an indication of the chemical character of a water as are the benthic invertebrates. Like chemical samples, phytoplankton samples are instantaneous, representing conditions at a single point. A sample collected at a specific site does not necessarily represent the river quality at that point, but rather the growth conditions of the reach upstream. For the New River at Calexico, the phytoplankton community was probably produced in Mexicali, whether above or below sewage outfall is uncertain. The nutrient enrichment from the sewage along with the high water temperature could lead to rapid proliferation of the phytoplankton as long as sufficient oxygen is present. The diel variations in DO concentration during the week of 

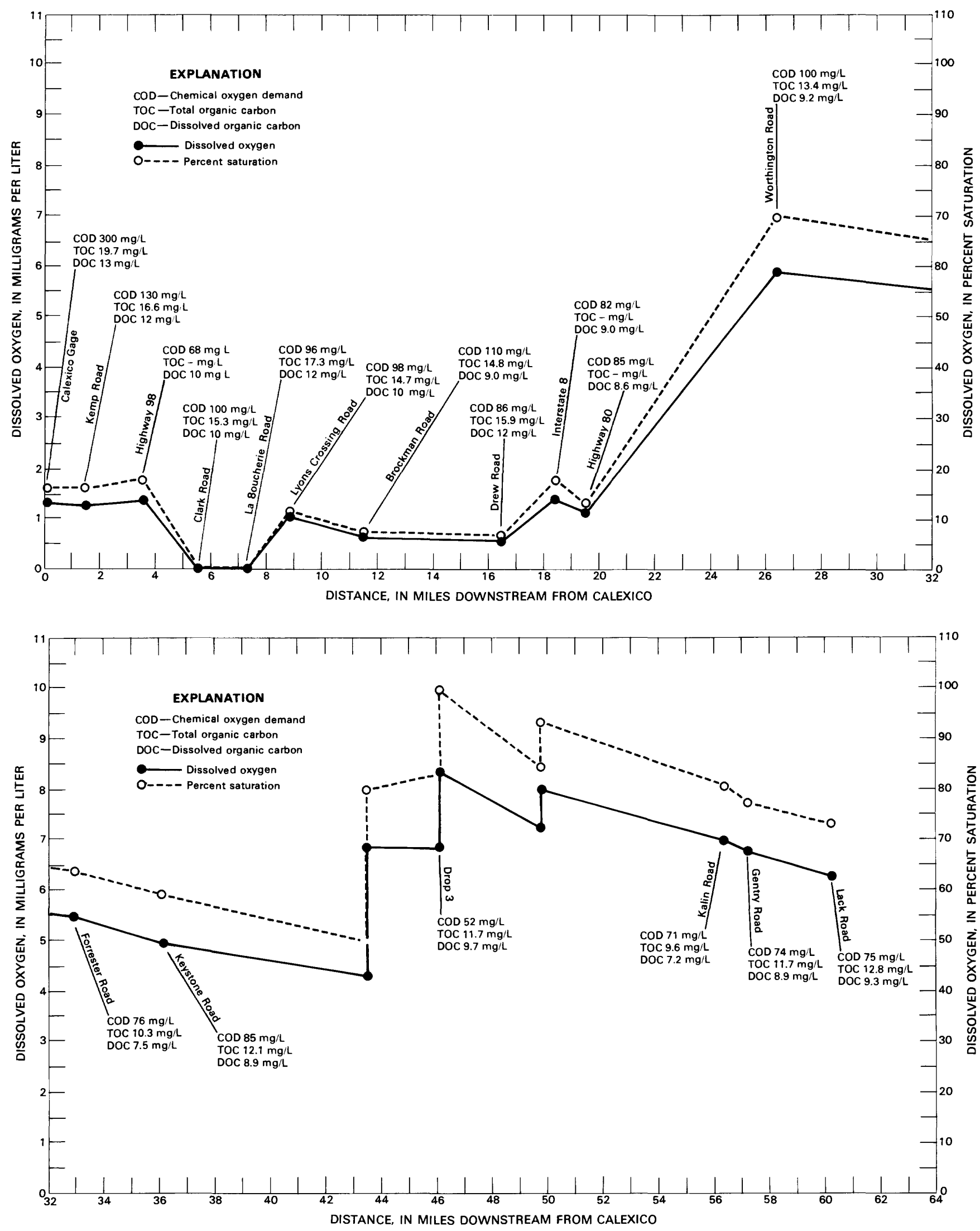

Figure 17. Dissolved-oxygen concentration, percent saturation, and selected chemical constituents at selected sites on the New River from Calexico to the Westmorland gage, May 10, 1978. 
Table 10. Loads of selected constituents for the composite sampling program

\begin{tabular}{|c|c|c|c|c|c|c|}
\hline & \multicolumn{6}{|c|}{ Load, in tons per day } \\
\hline & $\underset{26}{\mathrm{July}}$ & $\underset{17}{\text { Aug. }}$ & $\operatorname{Sept}_{26}$ & $\begin{array}{l}\text { Oct.1 } \\
23-27\end{array}$ & $\begin{array}{l}\text { Nov. } \\
13\end{array}$ & $\begin{array}{c}\text { Dec. } \\
8\end{array}$ \\
\hline & \multicolumn{6}{|c|}{1978} \\
\hline $\begin{array}{l}\text { Biochemical oxygen } \\
\text { demand }\end{array}$ & 9.5 & 15.1 & 133 & 21.6 & 18.5 & 24.4 \\
\hline $\begin{array}{l}\text { Chemical oxygen } \\
\text { demand }\end{array}$ & 34.5 & 51.6 & 51.2 & 109 & 85.6 & 104 \\
\hline $\begin{array}{l}\text { Total organic } \\
\text { carbon }\end{array}$ & 4.9 & 7.0 & 10.2 & 10.3 & 10.5 & 14.2 \\
\hline $\begin{array}{l}\text { Dissolved organic } \\
\text { carbon }\end{array}$ & 4.0 & 4.6 & 5.6 & 7.8 & 5.5 & 8.4 \\
\hline Total phosphorus & 0.40 & 0.53 & 0.53 & 0.80 & 0.68 & 1.45 \\
\hline Dissolved residue & 1780 & 2090 & 1560 & 2070 & 1690 & 1770 \\
\hline $\begin{array}{l}\text { Total organic plus } \\
\text { ammonium nitrogen }\end{array}$ & 2.0 & 1.9 & 2.7 & 3.5 & 3.0 & 3.2 \\
\hline
\end{tabular}

${ }^{1}$ Based on eight consecutive 12-hour composites (approximately 6 a.m.-6 p.m.) instead of one 12-hour composite.

April 17, 1978, indicate that there is a plant community, part of which would be represented by the phytoplankton. Downstream from Calexico, the DO concentration could be a limiting factor in growth and reproduction, as would be the sensitivity of the organisms to high organic concentrations. These two components, together with an increasing sediment concentration from the agricultural drainage that would reduce the depth through which photosynthesis occurs, results in decreasing densities of phytoplankton.

Densities were greatest at the first three sites on the New River (fig. 19 and table 12). The most downstream site at the Westmorland gage and the control site on the Alamo River had the smallest densities, with as little as one species present at each for the April sam- ples. In general, the population also decreased in downstream order, the highest observed concentration was $38,000 \mathrm{col} / \mathrm{mL}$ (colonies per milliliter) at Calexico in October, the smallest $820 \mathrm{col} / \mathrm{mL}$ in April at the Westmorland gage, and only $550 \mathrm{col} / \mathrm{mL}$ in April at the control site.

Many of the phytoplankton in the New River have been classified as pollution tolerant, according to criteria established in Palmer (1968). Organisms such as Oscillatoria, Euglena, Nitzschia, Chlamydomonus, Synedra, and Scenedesmus which dominated the communities in the New River are in the list of 10 most pollution-tolerant genera (Palmer, 1968). The Alamo River at outlet also contains some of these phytoplankton, but, except for Synedra, they are generally in lower concentrations than in the New River. The major limiting factor at both the Westmorland gage and at the control site is probably the high silt concentrations as mentioned earlier in this section.

\section{Bacteria}

To show the degree of fecal pollution of the New River, samples to determine the presence of fecal coliform and fecal streptococcal bacteria were collected at 16 sites during the profile of September 26-30, 1977.

\section{Potential Hazard}

The presence of fecal coliform and fecal streptococcal bacteria in an aquatic system means that fecal contamination has occurred and that the potential exists for a public health problem-the higher the number of indicator

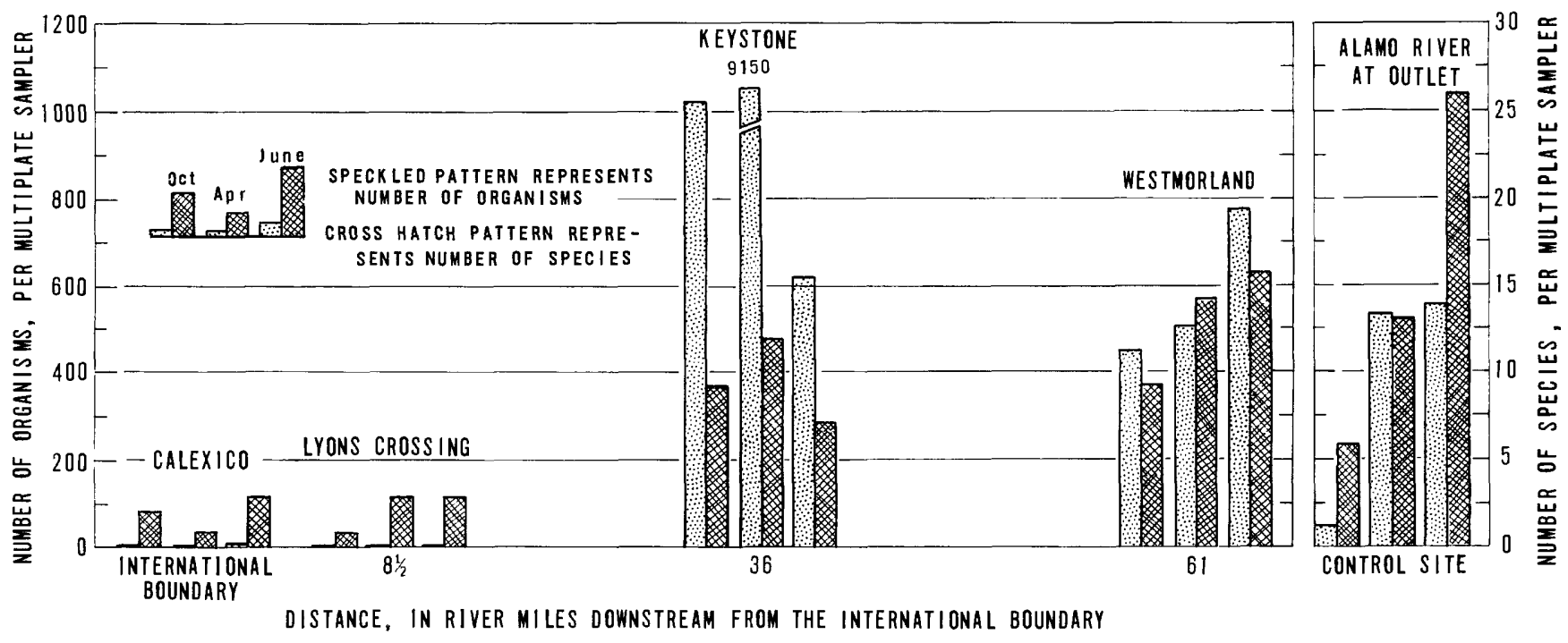

Figure 18. Number of benthic invertebrate species and organisms for selected sites on the New River and the control site on the Alamo River. 
Table 11. Phyla and genera of benthic invertebrate samples, from the New and Alamo Rivers [Leaders in columns listing generic names indicate absence of the organism on the specific date]

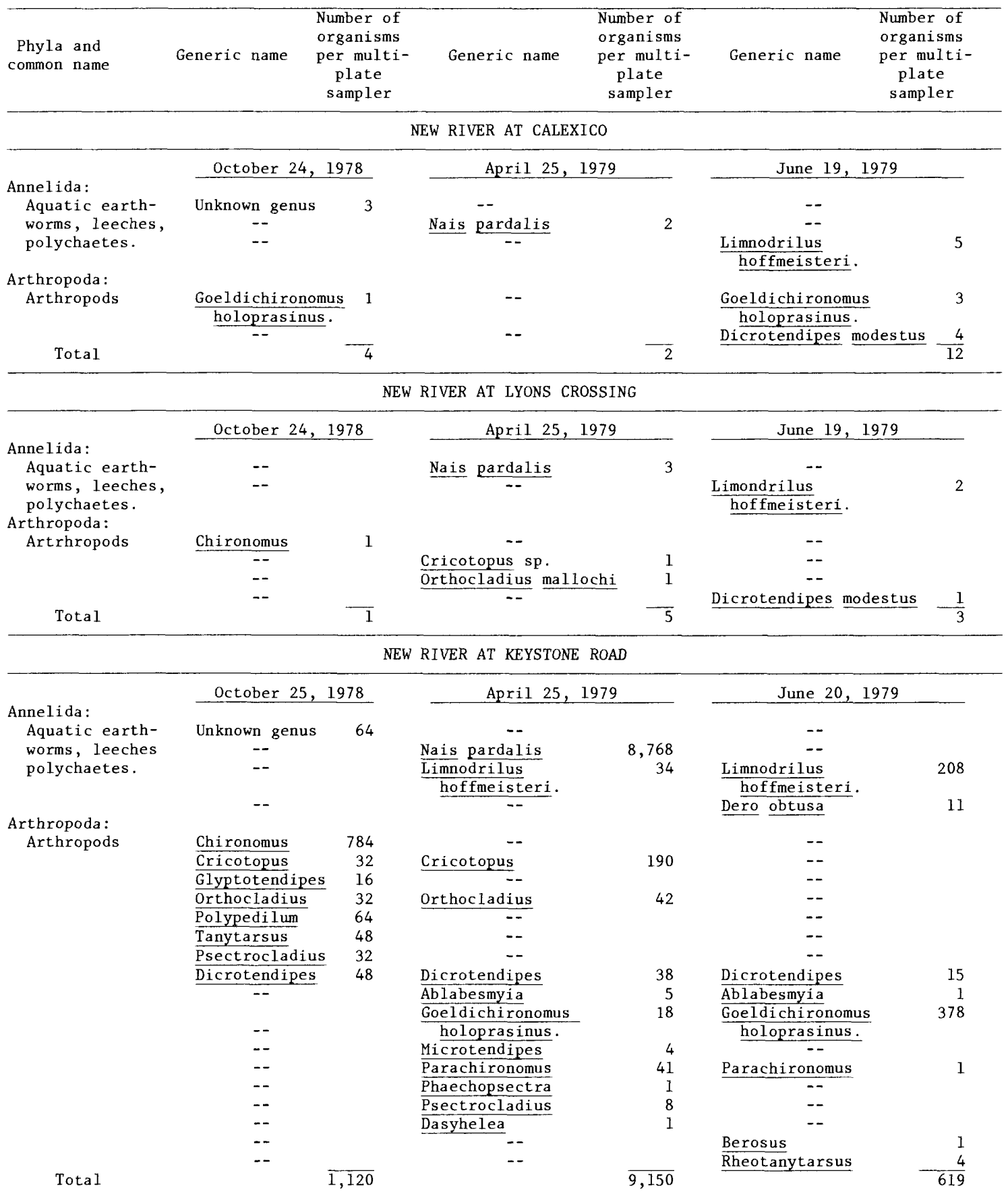

bacteria, the greater the health risk. Correlations between the density of indicator organisms and the occurrence of specific pathogens are not available except for the Salmonella group. Geldriech's study (1970) of the occurrence of Salmonella in freshwater indicated almost 32 percent positive detection of Salmonella when fecal coliform concentrations were $1-200 \mathrm{col} / 100 \mathrm{~mL}$ (colonies per $100 \mathrm{mil}-$ liliters). Detection of Salmonella increased to 83 percent for samples having fecal coliform concentrations of 201-
$1,000 \mathrm{col} / 100 \mathrm{~mL}$. For fecal coliform concentrations of 1,001-2,000 col/100 mL, more than 88 percent positive detection was found, and for concentrations above 2,000 , positive detection increased to nearly 98 percent. Salmonella cause diseases such as typhoid and paratyphoid fever and a variety of types of "food poisoning." Other diseases possibly resulting from fecal contamination are dysentery, cholera, hepatitis, and a wide range of parasitic infections. 
Table 11. Phyla and genera of benthic invertebrate samples, from the New and Alamo Rivers-Continued

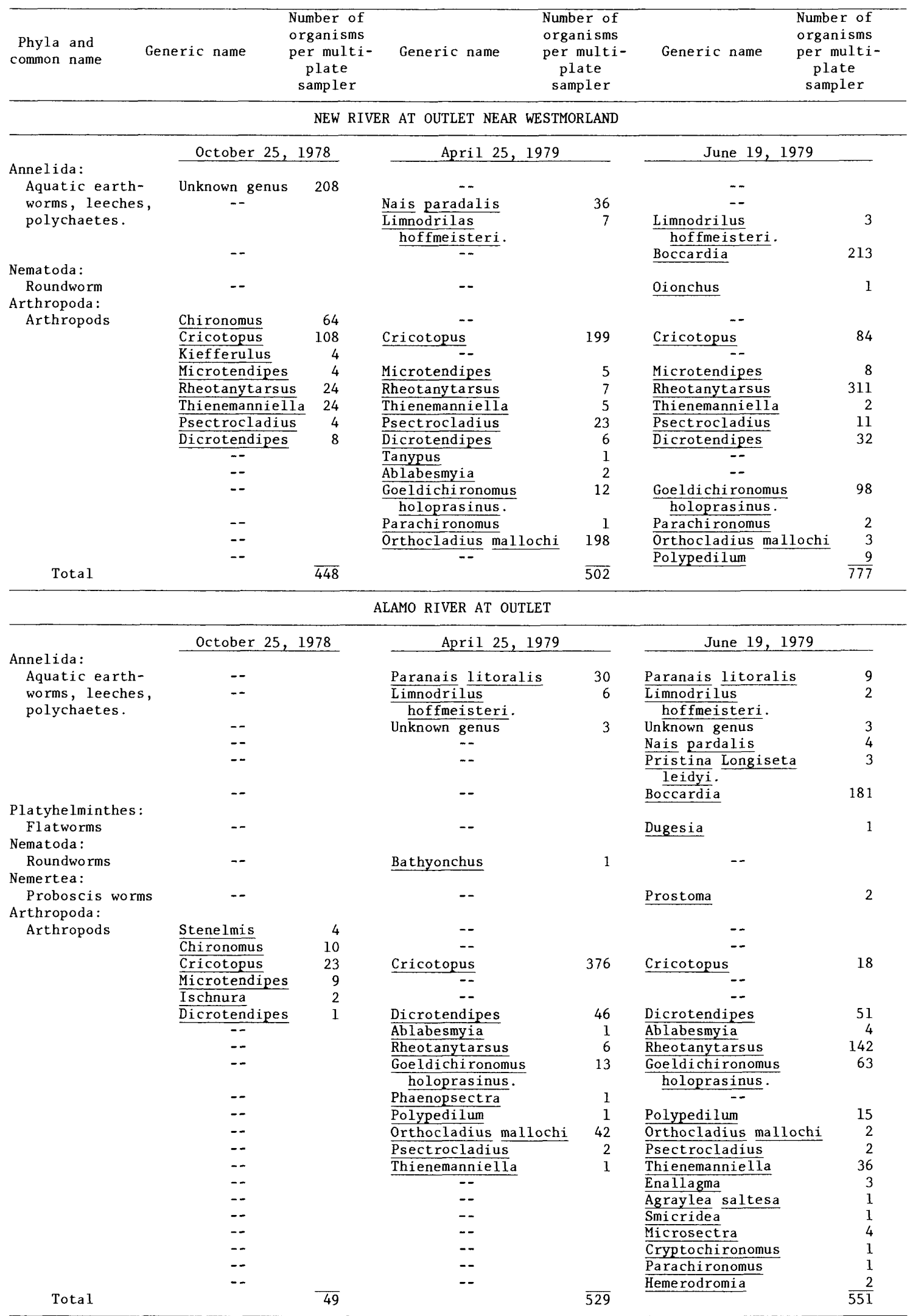




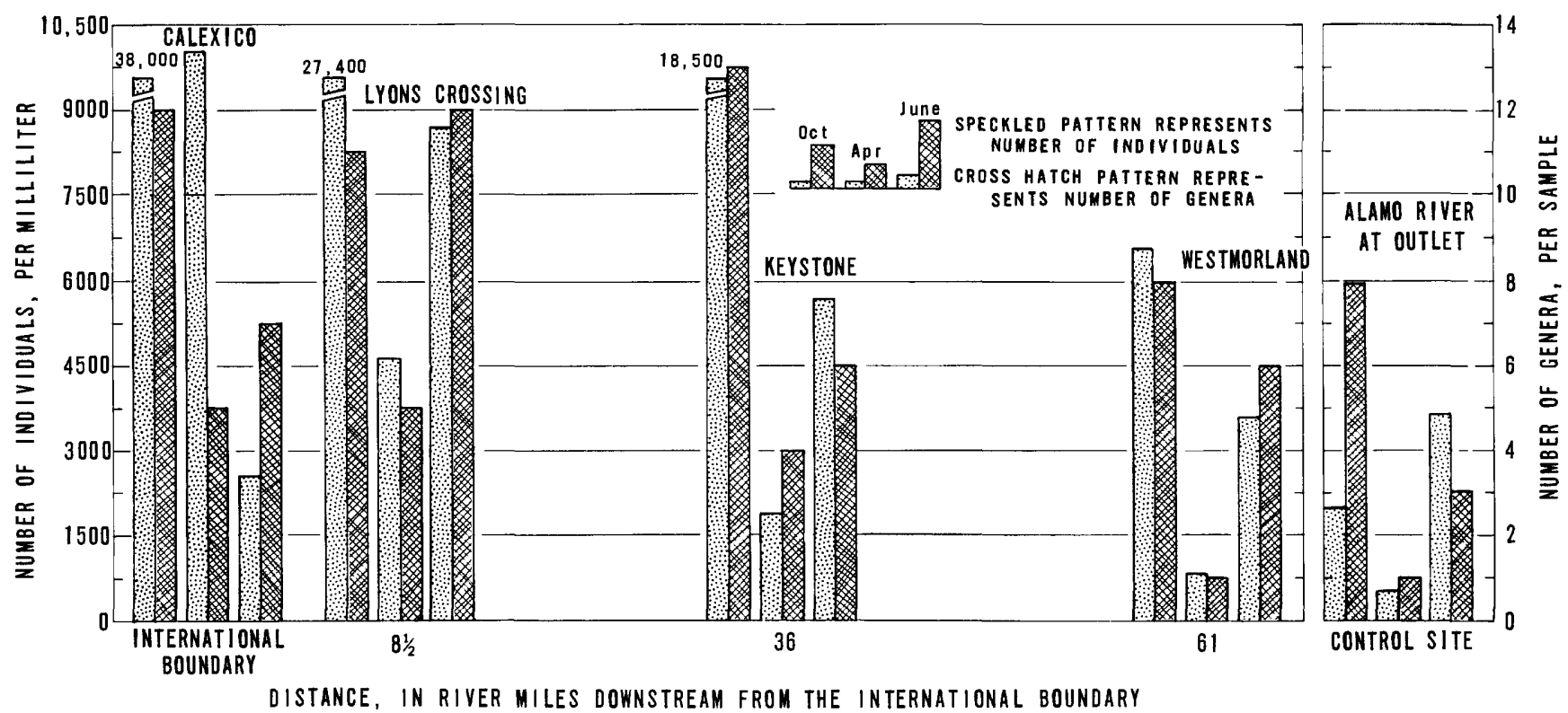

Figure 19. Number of phytoplankton genera and organisms for selected sites on the New River and the control site on the Alamo River.

\section{Observed Counts}

The highest fecal coliform concentration observed during the river profile was $2,800,000 \mathrm{col} / 100 \mathrm{~mL}$ at Brockman Road, 11.4 mi downstream from the international boundary. The corresponding fecal streptococcal concentration was $180,000 \mathrm{col} / 100 \mathrm{~mL}$. The lowest fecal coliform concentration, $1,000 \mathrm{col} / 100 \mathrm{~mL}$ (a non-ideal count), was observed at Lack Road, $60.2 \mathrm{mi}$ downstream from the boundary. The corresponding fecal streptococcal concentration was also $1,000 \mathrm{col} / 100 \mathrm{~mL}$ and a non-ideal count. There was no discernible pattern from site to site, although generally the concentrations near the mouth of the New River were lower than at the boundary, as expected with the die-off of organisms with time in the aquatic system. The absence of a pattern resulted from the compounding of variables, the distance and time from the source of contamination, the cyclic nature of domestic sewage discharge, and the time and direction of sample collection.

\section{SUMMARY AND CONCLUSIONS}

The New River crosses the international boundary and enters the United States at Calexico. Visual observation of the water at the Calexico gage is sufficient to determine that the New River is degraded by domestic sewage as well as by periodic slug discharges that appear to be of industrial origin. In order to quantify the chemical composition of this water and to identify the water-quality problems, a reconnaissance of the New River at Calexico was made May 9-13, 1977. Analysis of samples collected during the reconnaissance indicated excessive organic material as the major water-quality problem. Passage of three slugs of pollution were detected during this week of around-the-clock sampling. One of the slugs that was intensively sampled had TOC concentrations that ranged from 80 to $161 \mathrm{mg} / \mathrm{L}$, DOC concentrations that ranged from 34 to $42 \mathrm{mg} / \mathrm{L}$, and COD as high as $510 \mathrm{mg} / \mathrm{L}$. The $\mathrm{pH}$ increased from 7.6 prior to the arrival of the pollutant slug to a maximum of 8.8 , then decreased to 7.7 as the slug passed. In comparison, concentrations of COD, TOC, and DOC at the control site, Alamo River at Drop 9 near Holtville, were 72,11 , and $15 \mathrm{mg} / \mathrm{L}$, respectively. Although there is a significant amount of industry in Mexicali, no trace elements were found in concentrations that would indicate significant industrial pollution by these constituents.

A series of river profiles was made in which DO concentrations were measured at selected points between Calexico and the Salton Sea. These profiles showed a DO sag downstream from Calexico, with the length of the zone of depressed DO concentration varying seasonally. These seasonal variations correlated with changes in water temperature. At the Calexico gage, profiles measured during winter months had DO concentrations that approached saturation levels. The sag developed gradually, reaching minimum concentrations at Highway $80,19.5 \mathrm{mi}$ downstream. From Highway 80 downstream, recovery through reaeration and self-purification occurred, with DO concentrations in the lower reaches of the river being able to support a varied fish population. With the approach of summer, the DO concentration in the water crossing 
the international boundary became progressively lower. The water entering the United States via the New River was devoid of oxygen. The length of the depressed zone extended $26 \mathrm{mi}$ downstream , and even though some recovery was realized in the lower reaches of the river, the water quality was too poor to support a varied fish population.

Two diel studies were made during which DO concentrations were measured at five sites. During the first diel study, a slug of organic material was detected at the Calexico gage. The slug was detected both visually and by a sudden decrease in DO concentration as the water in the river became anaerobic. Movement of this slug downstream to Highway 80 took about 18 hours. Upon arrival of the slug at Highway 80 , the DO concentration plummeted to $0.1 \mathrm{mg} / \mathrm{L}$. At the other downstream sites, no significant variations in DO concentration were observed throughout the diel study. This diel study showed that degradation of water quality from slug discharges occurs as far downstream as $20 \mathrm{mi}$.

The second diel study was tied into the results of the time-of-travel investigation so that sampling at each site began with the arrival of the leading edge of the water sampled at the previous site. At the Calexico gage, the DO concentration presented a normal pattern of diel variations, similar to that of other eutrophic streams, except concentrations were considerably lower than normal. DO concentrations reached maximums during the afternoon and minimums in the early morning. At stations downstream from Lyons Crossing, no significant variations in DO concentration were observed.

The profile and diel phases of the study showed that DO concentrations in the upper reaches of the New River often fell below the $5.0 \mathrm{mg} / \mathrm{L}$ criterion established by EPA to maintain a varied fish population. In the lower reaches of the river, DO concentrations exceeded the 5.0 $\mathrm{mg} / \mathrm{L}$ criterion, except for a few instances in the summer, and were therefore able to support a varied fish population.

The movement and destination of organic material in the New River was also determined by the profile and diel phases of the study. The longitudinal profiles of September 26-30, 1977, and May 10, 1978, were the only profiles that involved the collection of samples for determination of organic concentrations. These two profiles showed organic concentrations to be about twice as high at the Calexico gage as at the Westmorland gage. The diel study made during the week of October 17, 1977 , showed the movement of a slug of organic material from Calexico to Highway 80 . Passage of the slug by the Calexico gage caused COD's to increase from 120 to 480 $\mathrm{mg} / \mathrm{L}$ and at Highway 80 , arrival of the slug caused the BOD to increase from 5.1 to $18.4 \mathrm{mg} / \mathrm{L}$. Downstream from Highway 80 , organic concentrations were generally lower as a result of both oxidation and dilution.
The composite sampling program provided an indication of the loads of selected constituents entering the United States via the New River and the magnitude of the effort that would be required to alleviate their effects on downstream water quality. Constituent loads for the eight consecutive 12-hour composite sampling programs in October 1978 were high, with COD averaging

$109 \mathrm{ton} / \mathrm{d}$, TOC $10.3 \mathrm{ton} / \mathrm{d}$, and dissolved residue 2.070 ton/d. These loads represent a significant stress on the self-cleansing processes of the New River and eventually the Salton Sea.

Because the river's biota must withstand the extremes in water quality as well as adapt to average conditions, they are excellent indicators of the environmental health of a river. Biological samples were collected at four sites on the New River and a control site on the Alamo River. The benthic-invertebrate data showed that the water at the first two sites, Calexico and Lyons Crossing, is of such poor quality that very little animal life can exist. At these two sites no more than three species and as few as one organism were present at any time. Downstream at Keystone Road, the quality of the water had improved although it would still be considered polluted. The diversity and the population increased. For the April sample, the population reached 9,150 organisms, of which 8,770 were of one species. At the Westmorland gage, the diversity and population indicated that the water quality was continuing to improve though it still would be considered moderately polluted. The control site on the Alamo River had the greatest diversity of organisms for the study. The June sample had 551 organisms distributed among 26 species with a greater number of phyla represented. The water at the control site, however, would still be considered mildly polluted. The biological diversity and the communitystructure of the benthic invertebrate population at the control site show by comparison that water quality in the New River suffers from the effects of the domestic and apparent industrial wastes observed at Calexico as far downstream as the Westmorland gage.

Phytoplankton samples showed that the greatest densities of organisms were at the first three sites. The most downstream site at the Westmorland gage and the control site on the Alamo River had the smallest densities. In general, the phytoplankton population decreased in downstream order. The high cell concentrations at Calexico are indicative of the water quality not at the Calexico gage but rather at some point upstream. The decreasing densities resulted from the increasing silt concentrations and the toxic products resulting from the anaerobic decomposition of organic matter. It might also be surmised that low and zero DO concentrations would accelerate the decline in phytoplankton densities. Although phytoplankton photosynthesize during daylight hours, they must respire during the nighttime, utilizing 
Table 12. Phyla and genera of phytoplankton samples from the New and Alamo Rivers

[Leaders in columns listing generic names indicate absence of the cells on the specific data]

\begin{tabular}{|c|c|c|c|c|c|c|}
\hline $\begin{array}{l}\text { Phyla and } \\
\text { common name }\end{array}$ & Generic name & $\begin{array}{l}\text { nber of } \\
\text { lls per } \\
\text { liliter }\end{array}$ & Generic name & $\begin{array}{l}\text { Number of } \\
\text { cells per } \\
\text { milliliter }\end{array}$ & Generic name & $\begin{array}{l}\text { mber of } \\
\text { lls per } \\
\text { liliter }\end{array}$ \\
\hline \multicolumn{7}{|c|}{ NEW RIVER AT CALEXICO } \\
\hline & \multicolumn{2}{|c|}{ October 24, 1978} & \multicolumn{2}{|c|}{ April 25, 1979} & \multicolumn{2}{|c|}{ June 20,1979} \\
\hline \multirow{5}{*}{$\begin{array}{l}\text { Chlorophyta: } \\
\text { Green algae }\end{array}$} & & & & & & \\
\hline & $\frac{\text { Ankistrodesmus }}{\text { Actinastrum }}$ & $\begin{array}{l}540 \\
580\end{array}$ & $\begin{array}{l}-- \\
--\end{array}$ & & $\begin{array}{l}-- \\
--\end{array}$ & \\
\hline & -- & & Chl amydomonas & 8,200 & -- & \\
\hline & -- & & $\overline{\text { Scenedesmus }}$ & 820 & Scenedesmus & 140 \\
\hline & -- & & -- & & $\overline{\text { Soclastrum }}$ & 140 \\
\hline \multirow{6}{*}{$\begin{array}{l}\text { Chrysophyta: } \\
\text { Golden algae }\end{array}$} & & & & & & \\
\hline & Chaetoceros & 220 & -- & & -- & \\
\hline & Cyclotella & 220 & -- & & -- & \\
\hline & Nitzschia & 36 & -- & & -- & \\
\hline & -- & & Coscinodiscus & 270 & Coscinodiscus & 140 \\
\hline & -- & & Synedra & 550 & Synedra & 550 \\
\hline \multirow{9}{*}{$\begin{array}{l}\text { Cyanophyta } \\
\text { Blue-green } \\
\text { algae. }\end{array}$} & & & & & & \\
\hline & Agmenellum & 25,000 & -- & & Agmenellum & 1,200 \\
\hline & $\overline{\text { Anacystis }}$ & 510 & -- & & -- & \\
\hline & Cylindrospermum & 1,100 & -- & & -- & \\
\hline & Lyngbya & 5,500 & -- & & -- & \\
\hline & Oscillatoria & 4,700 & -- & & Oscillatoria & 270 \\
\hline & Spirulina & 36 & -- & & -- & \\
\hline & Raphidiopsis & 180 & -- & & -- & \\
\hline & -- & & -- & & Aphanzomenon & 140 \\
\hline \multirow{3}{*}{$\begin{array}{c}\text { Euglenophyta: } \\
\text { Euglenoids } \\
\text { Total }\end{array}$} & & & & & & \\
\hline & -- & $\overline{38,600}$ & Euglena & $\frac{550}{10,400}$ & -- & $\overline{2,600}$ \\
\hline & \multicolumn{4}{|c|}{ NEW RIVER AT LYONS CROSSING } & & \\
\hline & \multicolumn{2}{|c|}{ October 24, 1978} & \multicolumn{2}{|c|}{ April 25, 1979} & \multicolumn{2}{|c|}{ June 20, 1979} \\
\hline \multirow[t]{3}{*}{$\begin{array}{l}\text { Chlorophyta: } \\
\text { Green algae }\end{array}$} & $\frac{\text { Ankistrodesmus }}{\text { Oocustis }}$ & 97 & Ankistrodesmus & 270 & Ankistrodesmus & 550 \\
\hline & Scenedesmus & 270 & Scenedesmus & 1,100 & Scenedesmus & 550 \\
\hline & -- & & Chlamydomonas & 2,200 & Chlamydomonas & 410 \\
\hline \multirow{8}{*}{$\begin{array}{l}\text { Chrysophyta: } \\
\text { Golden algae }\end{array}$} & & & & & & \\
\hline & Chaetoceros & 97 & -- & & -- & \\
\hline & $\overline{\text { Cyclotella }}$ & 97 & -- & & Cyclotella & 550 \\
\hline & $\frac{\text { Nitzschia }}{\text { Nita }}$ & 24 & -- & & - & \\
\hline & -- & & Coscinodiscus & 550 & -- & \\
\hline & -- & & -- & & Arachnochloris & 270 \\
\hline & -- & & -- & & Synedra & 690 \\
\hline & -- & & -- & & Navicula & 960 \\
\hline \multicolumn{7}{|l|}{ Cyanophyta: } \\
\hline Blue-green & Agmenellum & 16,000 & -- & & Agmenellum & 1,100 \\
\hline \multirow{5}{*}{ algae. } & Anacystis & 1,200 & -- & & Anacystia & 2,100 \\
\hline & Cylindrospermum & 190 & -- & & $\overline{--}$ & \\
\hline & Arthrospira & 850 & -- & & -- & \\
\hline & Lyngbya & 2,200 & -- & & -- & \\
\hline & Oscillatoria & 6,400 & Oscillatoria & 550 & Oscillatoria & 1,200 \\
\hline \multirow{3}{*}{$\begin{array}{c}\text { Euglenophyta: } \\
\text { Euglenoids } \\
\text { Total }\end{array}$} & & & & & & \\
\hline & -- & & -- & & Euglena & 140 \\
\hline & -- & $\overline{27,400}$ & -- & $\overline{4,700}$ & Cryptomonas & $\frac{140}{8,700}$ \\
\hline
\end{tabular}

the DO available in their environment. For the upper reaches of the New River, the environment provides little or no DO, thereby stressing the phytoplankton and resulting in a decrease in their densities. Many of the phytoplankton found in the New River have been classified as pollution-tolerant species; the control site on the Alamo River had fewer of these species.

During the profile of September 26-30, 1977, samples were collected to determine the presence of fecal coliform and fecal streptococcal bacteria. The presence of these two groups of enteric bacteria in an aquatic system means that fecal contamination has occurred and that the potential exists for a public health problem. Concentrations near the mouth of the New River were lower than at the boundary, although there was no discernible pattern from sample to sample. The highest observed fecal coliform concentration of $2,800,000 \mathrm{col} / 100 \mathrm{~mL}$ occurred $11.4 \mathrm{mi}$ downstream from the boundary at Brockman Road

This study has documented water-quality conditions 
Table 12. Phyla and genera of phytoplankton samples from the New and Alamo Rivers-Continued

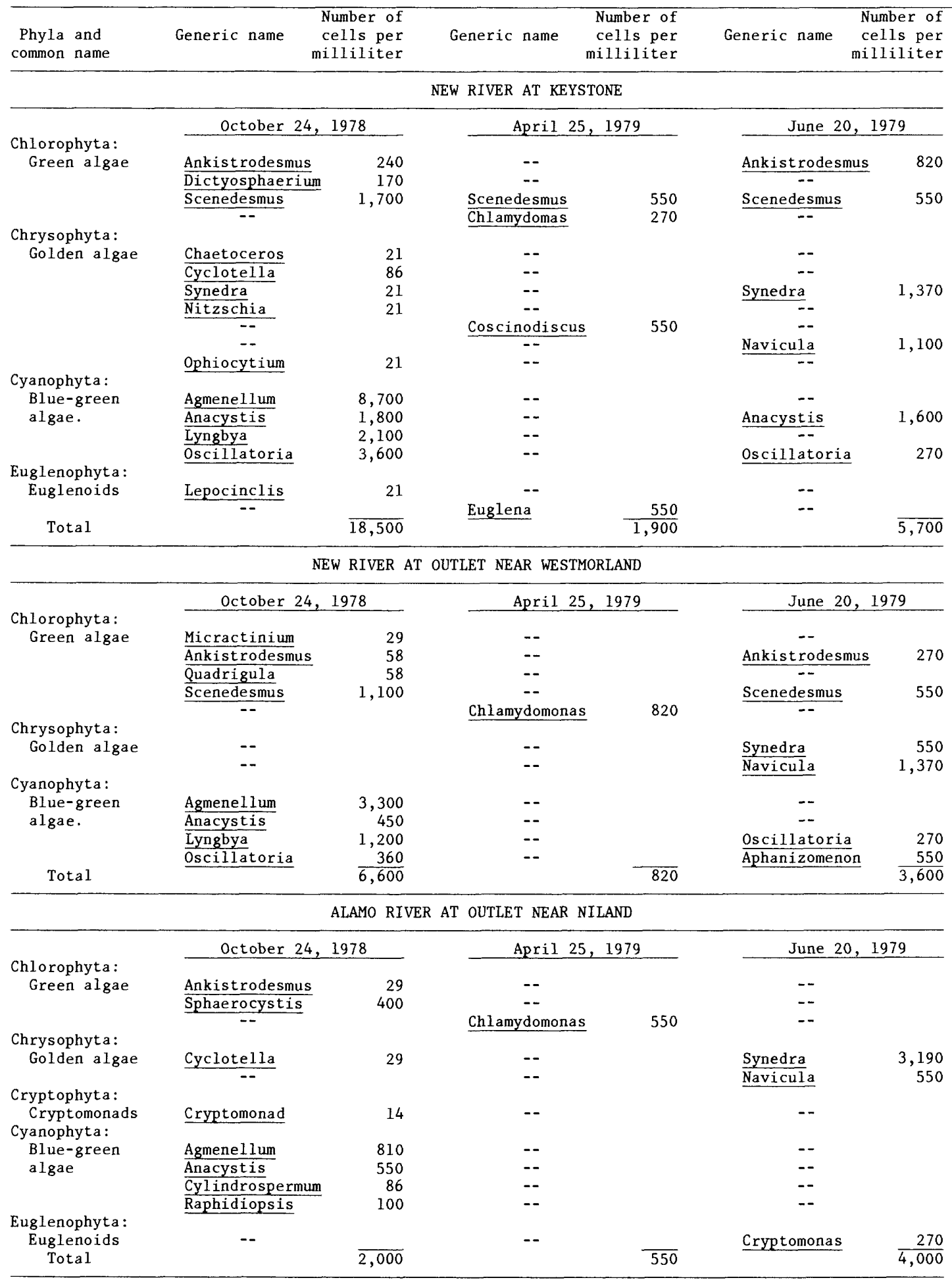

in the New River. High bacteriai and organic material concentrations were found in the water crossing the international boundary at Calexico. The effects of this organic matter on downstream water quality are so noxious that upper reaches of the river are unfit not only for human contact, but severely restrict aquatic organisms. 


\section{SELECTED REFERENCES}

American Public Health Association, American Water Works Association, and Water Pollution Control Federation, 1976, Standard methods for the examination of water and wastewater, 14th ed: $1193 \mathrm{p}$.

Blake, W. P., 1857, Geological report, Exploration and surveys for a railroad route from the Mississippi River to the Pacific Ocean, routes in California to connect with routes near the thirty-fifth and thirty-second parallels, explored by Lieutenant R. S. Williamson, Corps of Topographic Engineers, in 1853: Washington D.C., War Department 83106, p. 233 248.

Geldreich, E. E., 1970, Applying bacteriological parameters to recreational water quality: Journal of American Water Works Association, v. 62, no. 2, p. 113-120.

Goerlitz, D. G., and Brown, Eugene, 1972, Methods for analysis of organic substances in water: U.S. Geological Survey Techniques of Water-Resources Investigations, Book 5, Chapter A3, $40 \mathrm{p}$.

Greeson, P. E., Ehlke, T. A., Irwin, G. A., Lium, B. W., and Slack, K. V., eds., 1977, Methods for collection and analysis of aquatic biological and microbiological samples (revised edition): U.S. Geological Survey Techniques for Water-Resources Investigations, Book 5, Chapter A4, 332 p.

Klein, Louis, 1962, River pollution II. Causes and effects: London, Butterworths, $432 \mathrm{p}$

National Academy of Sciences, National Academy of Engineering, 1973 [1974], Water quality criteria, 1972: U.S. Environmental Protection Agency, EPA R3-73-033, 594 p.
Palmer, C. M., 1968, A composite rating of algae tolerating organic pollution, in Algae as indicator organisms-a symposium: Phycological Society of America and Botanical Society of America, Phycological Section, Columbus, Ohio, September 1968, p. 78182.

Phelps, E. B., 1944, Stream sanitation: New York, Wiley, 263 p.

Rathbun, R. E., 1977, Reaeration coefficient of streams-Stateof-the-art: American Society of Civil Engineers Proceedings, Journal of Hydraulics Division, v. 103, no. HY4, p. 409421 .

Skougstad, M. W., Fishman, M. J., Friedman, L. C., Erdmann, D. E., and Duncan, S. S., eds., 1979, Methods for determination of inorganic substances in water and fluvial sediments: U.S. Geological Survey Techniques of Water-Resources Investigations, Book 5, Chapter A1, $626 \mathrm{p}$.

Stumm, Werner, and Morgan, J. J., 1970, Aquatic chemistry: Wiley-Interscience, $581 \mathrm{p}$.

Thatcher, L. L., Janzer, V. J., and Edwards, K. W., 1977, Methods for determination of radioactive substances in water and fluvial sediments: U.S. Geological Survey Techniques of Water-Resources Investigations, Book 5, Chapter A5, $95 \mathrm{p}$.

U.S. Department of the lnterior and the Resources Agency of California, 1974, Salton Sea project, Federal-State feasibility report: v. 3, 185 p.

U.S. Environmental Protection Agency, 1976 [1977], Quality criteria for water: Washington, U.S. Government Printing Office, $256 \mathrm{p}$

Velz, C. J., 1970, Applied stream sanitation: Wiley-Interscience, $619 \mathrm{p}$

\section{FACTORS FOR CONVERTING INCH-POUND UNITS TO INTERNATIONAL SYSTEM OF UNITS (SI)}

The inch-pound system is used in this report. For readers who prefer metric units, the conversion factors for the terms used in this report are listed below.

\begin{tabular}{lll}
\hline Multiply & By & To obtain \\
\hline acre & 0.4047 & ha (hectare) \\
acre-ft/yr (acre-foot per year) & 0.001233 & $\mathrm{hm}^{3} / \mathrm{a}$ (cubic hectometer per annum) \\
$\mathrm{ft}$ (foot) & 0.3048 & $\mathrm{~m}$ (meter) \\
$\mathrm{ft} / \mathrm{s}$ (foot per second) & 0.3048 & $\mathrm{~m} / \mathrm{s}$ (meter per second) \\
$\mathrm{ft} / \mathrm{s}$ (cubic foot per second) & 0.02832 & $\mathrm{~m}^{3} / \mathrm{s}$ (cubic meter per second) \\
$\mathrm{lb}$ (pound) & 0.45 & $\mathrm{~kg}$ (kilogram) \\
$\mathrm{mi}$ (mile) & 1.609 & $\mathrm{~km}$ (kilometer) \\
$\mathrm{mi} / \mathrm{h}$ (mile per hour) & 1.609 & $\mathrm{~km} / \mathrm{h}$ (kilometer per hour) \\
ton/d (ton per day) & 0.9072 & $\mathrm{Mg} / \mathrm{d}$ (megagram per day) \\
$\mu \mathrm{mho}$ (micromho) & 1.000 & $\mu \mathrm{S}$ (microsiemens) \\
$\mu \mathrm{mho/cm}$ (micromho per centimeter) & 1.000 & $\mu \mathrm{S} / \mathrm{cm}$ (microsiemens per centimeter)
\end{tabular}

\footnotetext{
Abbreviations used:

BOD (biochemical oxygen demand)

${ }^{\circ} \mathrm{C}$ (degree Celsius)

COD (chemical oxygen demand)

$\mathrm{col} / 100 \mathrm{~mL}$ (colonies per 100 milliliters)

DOC (dissolved organic carbon)

DO (dissolved oxygen)
}

JTU (Jackson turbidity unit) $\mu \mathrm{g} / \mathrm{L}$ or UG/L (microgram per liter) $\mathrm{mg} / \mathrm{L}$ or $\mathrm{MG} / \mathrm{L}$ (milligram per liter) $\mathrm{mL}$ (milliliter) $\mathrm{pCi} / \mathrm{L}$ or $\mathrm{PC} / \mathrm{L}$ (picocurie per liter) TOC (total organic carbon) 